WHC-EP-0172

Revision 1

\title{
Inventory of Chemicals Used at Hanford Site Production Plants and Support Operations (1944-1980)
}

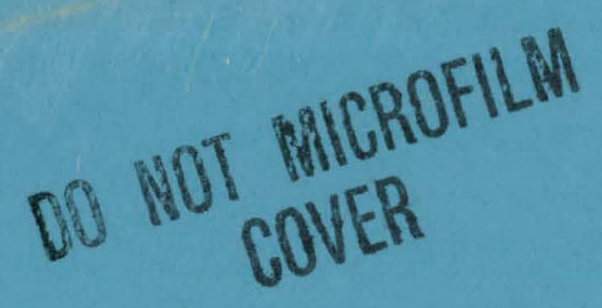

Prepared for the U.S. Department of Energy Assistant Secretary for Defense Programs

W. Westinghouse

Hanford Operations and Engineering Contractor for the

U.S. Department of Energy under Contract DE-AC06-87RL10930 


\section{DISCLAIMER}

This report was prepared as an account of work sponsored by an agency of the United States Government. Neither the United States Government nor any agency Thereof, nor any of their employees, makes any warranty, express or implied, or assumes any legal liability or responsibility for the accuracy, completeness, or usefulness of any information, apparatus, product, or process disclosed, or represents that its use would not infringe privately owned rights. Reference herein to any specific commercial product, process, or service by trade name, trademark, manufacturer, or otherwise does not necessarily constitute or imply its endorsement, recommendation, or favoring by the United States Government or any agency thereof. The views and opinions of authors expressed herein do not necessarily state or reflect those of the United States Government or any agency thereof. 


\section{DISCLAIMER}

Portions of this document may be illegible in electronic image products. Images are produced from the best available original document. 


\section{DISCLAIMER}

This report was prepared as an account of work sponsored by an agency of the United States Government. Neither the United States Government nor any agency thereof, nor any of their employees, nor any of their contractors, subcontractors or their employees, makes any warranty, expressed or implied, or assumes any legal liability or responsibility for the accuracy, completeness, or any third party's use or the results of such use of any information, apparatus, product, or process disclosed, or represents that its use would not infringe privately owned rights. Reference herein to any specific commercial product, process, or service by trade name, trademark, manufacturer, or otherwise, does not necessarily constitute or imply its endorsement, recommendation, or favoring by the United States Government or any agency thereof or its contractors or subcontractors. The views and opinions of authors expressed herein do not necessarily state or reflect those of the United States Government or any agency thereof.

\section{Available from}

U.S. Department of Commerce

National Technical Information Service

5285 Port Royal Road

Springtield, VA 22161

NTIS Price Codes:

Microfiche Copy: A01

Printed Copy:

Price Price

Pages Codes Pages Codes

$001.025 \quad \mathrm{A02} \quad 301-325 \quad$ A14

$026.050 \quad A 03 \quad 326-350$ A15

$051.075 \quad A 04 \quad 351-375 \quad$ A16

076-100 A05 $376-400 \quad$ A17

$\begin{array}{lll}101-125 & \text { A06 } & 401-425\end{array}$

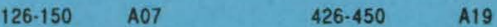

$151-175$ A08
$176-200$

$\begin{array}{lll}176-200 & \text { A09 } & 476.500\end{array}$

201.225 A10 $\quad 501.525 \quad$ A22

226-250 A11 $\quad 526.550 \quad$ A23

251275 A12 551.575 A24

$276.300 \quad A 13 \quad 576-600 \quad$ A25

Printed in the United States of America

DISCLM-1.CHP (2-89) 


\section{Inventory of Chemicals Used at Hanford Site Production Plants and Support Operations (1944-1980)}

M. J. Klem

Date Published

April 1990

Prepared for the U.S. Department of Energy Assistant Secretary for Defense Programs

\section{(2) Westinghouse $\begin{aligned} & \text { P.O. Box } 1970 \\ & \text { Hanford Company }\end{aligned}$}

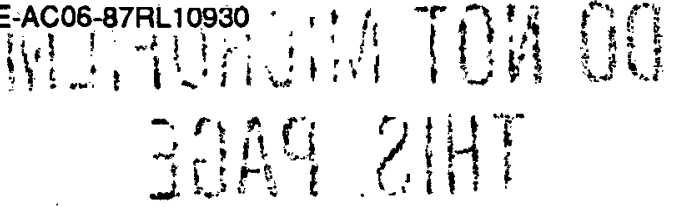




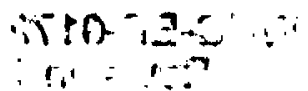

This page intentionally left blank.

\section{DO NOT MICROFILM THIS PAGE}

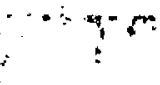




\title{
WESTINGHOUSE HANFORD COMPANY \\ INVENTORY OF CHEMICALS USED AT HANFORD \\ SITE PRODUCTION PLANTS AND SUPPORT \\ OPERATIONS (1944-1980)
}

\author{
M. J. Klem \\ Westinghouse Hanford Company
}

\begin{abstract}
A complete list of chemicals used in the production facilities and support operations of the U.S. Department of Energy Hanford Site is presented to aid development of plans for characterizing the radioactive liquid chemical wastes stored in the 149 single-shell tanks. The complete chemical list is compared to the list provided by the regulatory agencies to identify hazardous chemicals stored in the single-shell tanks. A reduced list has been developed by others and is used to identify the chemical constituents for analysis in the Waste Characterization Plan for the Hanford Site Single-Shell Tanks. The chemical list is based on chemical process flowsheets, essential material consumption records, letters, reports, and other historical data.
\end{abstract}


WHC-EP-0172 Revision 1

This page intentionally left blank.

\section{DO NOT MICROFILM \\ THIS PAGE}

ix 
WHC-EP-0172 Revision 1

\section{LIST OF TERMS}

AEA CERCLA

DST

DVB

Ecology

HDW-EIS

HWMA

IX

OES/TLV

OSHA

RCRA

SST

WAC
Atomic Energy Act

Comprehensive Environmental Response, Compensation, and Liability Act

double-shell tank

divinyl benzene

Washington State Department of Ecology

Hanford Defense Waste-Environmental Impact Statement

Hazardous Waste Management Act

ion exchange

Occupational Exposure Standards/Threshold Limit Values.

Occupational Safety and Health Administration

Resource Conservation and Recovery Act

single-shell tank

Washington Administrative Code

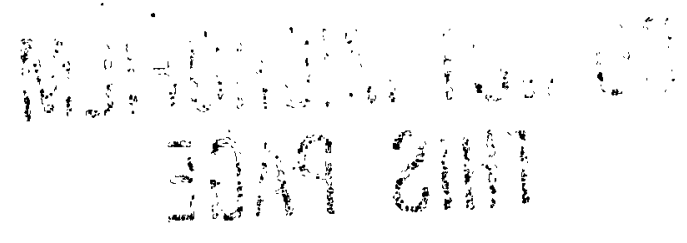

v/vi 
WHC-EP-0172 Revision 1

This page intentionally left blank.

\section{DO NOT MICROFILM \\ THIS PAGE}

l 
WHC-EP-0172 Revision 1

\section{CONTENTS}

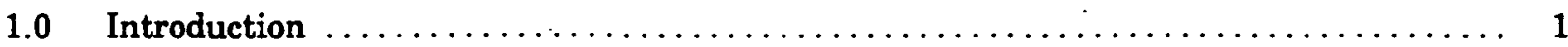

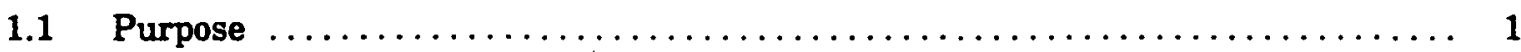

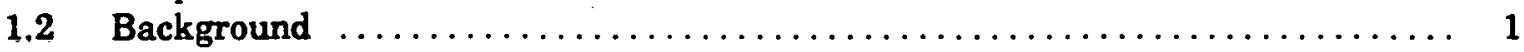

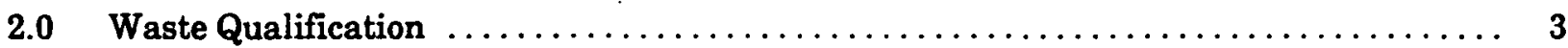

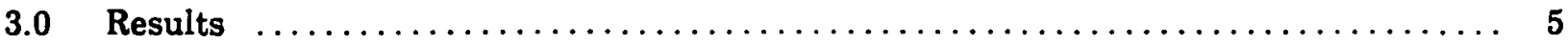

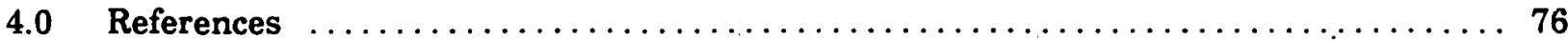




\section{LIST OF TABLES}

1. Summary List of Chemicals Used at Hanford Site Production Plants and Support Operations that Stored Waste in the Single-Shell Tanks (1944-1980) $\ldots \quad 6$

2. N Reactor Decontamination (100 Area) (1972-1976) $\ldots \ldots \ldots \ldots \ldots \ldots \ldots . \ldots 22$

3. PUREX Plant (A Plant): Fuel Reprocessing (200 East Area) (1955-1972)

4. PUREX Plant (A Plant): Analytical Laboratory (200 East Area) (1955-1972)

5. PUREX Plant (A Plant): Fission Product Recovery (200 East Area) (1960-1966)

6. B Plant: Fuel Reprocessing (200 East Area) (1945-1952) 27

7. B Plant: Strontium Recovery and Waste Fractionization (200 East Area) (1965-1976)

8. B Plant: Waste Encapsulation (200 East Area) $(1974-1976) \ldots \ldots \ldots \ldots \ldots \ldots$

9. Semiworks Pilot Plant (200 East Area) (1955-1967) $\ldots \ldots \ldots \ldots \ldots \ldots \ldots \ldots$

10. REDOX Plant (S Plant): Fuel Reprocessing (200 West Area) (1951-1967)

11. REDOX Plant (S Plant): Analytical Laboratory (200 West Area) (1951-1976)

12. T Plant: Fuel Reprocessing (200 West Area) $(1944-1956) \quad \ldots \ldots \ldots \ldots \ldots \ldots . \ldots 7$

13. T Plant: In-Plant Scavenging (200 West Area) (1955) $\ldots \ldots \ldots \ldots \ldots \ldots \ldots \ldots$

14. 221-T Plant: Containment Systems Experiment

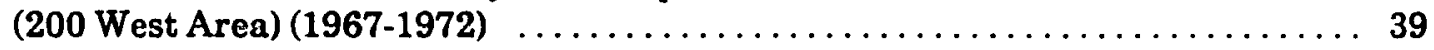

15. T Plant: Containment Systems Test Facility (200 West Area) (1977)

16. 221-T Plant: Equipment Decontamination (200 West Area) (1964-1980)

17. U Plant: $\left(\mathrm{UO}_{3}\right)$ Uranium Conversion (200 West Area) $(1952-1971) \ldots \ldots \ldots \ldots 44$

18. U Plant: Uranium Recovery and In-Plant Scavenging

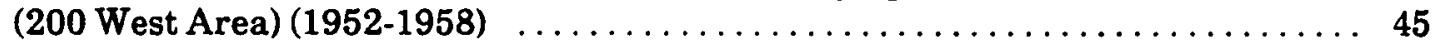

19. U Plant: Analytical Laboratory (200 West Area) (1952-1958) . . . . . . . 46 
20. U Plant: Equipment Decontamination (200 West Area) $(1961-1963) \ldots \ldots \ldots .47$

21. Plutonium Finishing Plant ( $\mathrm{Z}$ Plant): Plutonium Conversion to Oxide

22. Plutonium Finishing Plant (Z Plant): Plutonium Reclamation and Waste Treatment (200 West Area) (1973-1976)

23. Plutonium Finishing Plant (Z Plant): Analytical Laboratory

(200 West Area) (1973-1976)

24. Tank Farms (200 Areas): Equipment Decontamination and

Waste Evaporation/Solidification (1944-1980)

25. Tank Farms (200 Areas): Waste Scavenging and Sludge

Sluicing/Dissolution (1952-1976)

26. Building 242 (200 East Area): Irradiation Rupture

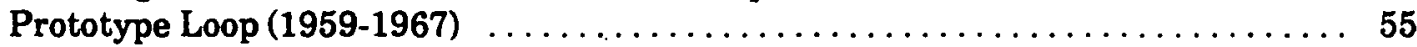

27. Building 324: Chemical Engineering Hot Cells (1966-1976) $\ldots \ldots \ldots \ldots \ldots \ldots$

28. Building 325: Analytical Laboratory (1953-1976) $\ldots \ldots \ldots \ldots \ldots \ldots \ldots \ldots$

29. Building 325: Westinghouse Hanford Company Development

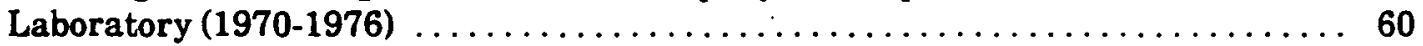

30. Building 325: Pacific Northwest Laboratory Development and

Production Laboratory (1955-1976)

31. Building 326: Electropolishing of Irradiated Alloy for

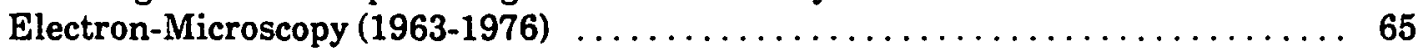

32. Building 326: Metallography Laboratory $(1955-1976) \ldots \ldots \ldots \ldots \ldots \ldots 6$

33. Building 327: Post-Irradiation Testing

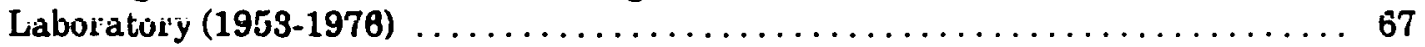

34. Building 329: Radiochemical Laboratory (1952-1976) $\ldots \ldots \ldots \ldots \ldots \ldots \ldots \ldots$

35. Building 340: Radioactive Liquid Waste Storage and

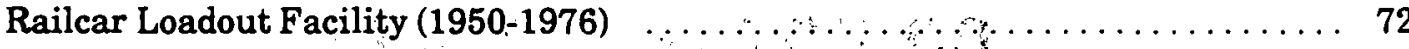

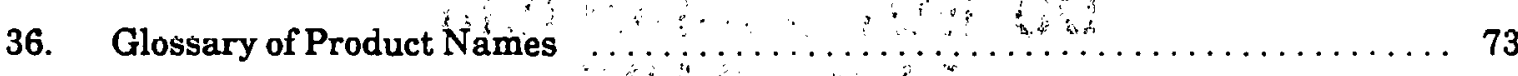


WHC-EP-0172 Revision 1

This page intentionally left blank.

\section{DO NOT MICROFILM THIS PAGE}

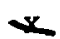




\section{INVENTORY OF CHEMICALS USED AT HANFORD SITE PRODUCTION PLANTS AND SUPPORT OPERATIONS (1944-1980)}

\subsection{INTRODUCTION}

\subsection{PURPOSE}

This document contains a comprehensive list of the nonradioactive chemicals used by production plants and support operation activities that stored mixed waste in single-shell tanks (SST) between 1944 and 1980 at the U.S. Department of Energy Hanford Site. This information has been used to develop a reduced list of chemical constituents for analysis in the Waste Characterization Plan for the Hanford Site Single-Shell Tanks. 1

\subsection{BACKGROUND}

Radioactive wastes on the Hanford Site have been generated since 1944 as part of the program required to support national defense activities. Liquid radioactive and chemical waste from the nuclear materials production and support activities were transferred to underground, reinforced concrete, steel-lined tanks (commonly referred to as SSTs) for storage. The SSTs were used for storage of several types of liquid waste before 1971. The tank-in-tank design [commonly referred to as a double-shell tank (DST)] was designed, fabricated, and then placed into service beginning in 1971. The DSTs have monitoring equipment to detect leakage from the inner shell and the capability to pump leaked waste from the space between the two steel shells. Since November 1980, DSTs have been used exclusively for receipt of new waste.

A total of 149 SSTs with capacities ranging from 55,000 to $1 \mathrm{Mgal}$ each were constructed on the 200 Area plateau of the Hanford Site between 1943 and $1964 .{ }^{2}$ Waste from several of the SSTs was retrieved in the 1950's for extraction of uranium and in the 1960's and 1970's for extraction of cesium and strontium fission products. During the 1970 s, the volume of liquid waste stored in these tanks was reduced by evaporating the liquids, leaving moist sludge and saltcake in the SSTs. The evaporation effort also served to reduce the environmental impacts of potential releases from the tanks by minimizing the volume of drainable liquids available to contaminate the soil around the tanks. The SSTs now contain about $37 \mathrm{Mgal}$ of saltcake, sludge, interstitial, and nonpumpable supernatant liquids.

A large variety of chemicals were used in the production plants and support operations that generated the SST waste. Several of these chemicals are currently classified as hazardous. At the time the wastes were generated, there were no definitions or regulations for dangerous or mixed wastes. In May 1987, DOE issued a mixed byproduct waste ruling stating that the hazardous component of the waste is regulated by the Resource Conservation and Recovery Act (RCRA). 3 In November 1987, the Environmental Protection Agency authorized the Washington State Department of Ecology (Ecology) to regulate the hazardous constituents of mixed wastes. 4

Activities supporting final disposal of the SST wastes are underway. The Hanford Defense Waste-Environmental Impact Statement (HDW-EIS) Record of Decision and the Hanford Federal Facility Agreement and Consent Order commit to additional development and evaluation before making a final disposal decision on SST waste. 5,6 A key part of this evaluation is characterization of 
WHC-EP-0172 Revision 1

the waste according to regulatory requirements. The hazardous constituents are subject to regulation under the RCRA 7 and Washington State Hazardous Waste Management Act (HWMA) 8 while the radioactive constituents of the mixed waste are subject to regulation under the Atomic Energy Act (AEA). 9 Statutes other than RCRA, HWMA, and AEA may provide guidance in the waste characterization effort.

The waste contained in the SSTs will be characterized to identify the risks to the public and to the environment from the contemplated disposal actions. In particular, hazardous chemicals must be quantified to support additional environmental protection activities. Previous sampling efforts have been focused largely on determining the amounts of radionuclides stored in the SSTs; thus, identification of hazardous chemicals has not been fully addressed. The waste characterization plan addresses both radionuclides and hazardous chemicals and has been submitted to Ecology for approval.

This document identifies the nonradioactive chemicals used by the production plants and support operation activities that transferred mixed waste to the SSTs. This information has been used to develop a reduced list of chemical constituents for the SST waste characterization plan. The inventory list of chemicals is compared to the list provided by the regulatory agencies to identify hazardous chemicals that may be stored in the SSTs.

The tables list the chemicals that were added during processing and support operations. It is recognized that chemical reactions (e.g., oxidation-reduction, neutralization, precipitation, radiolysis) have converted many of these chemicals into different chemical compounds. These tables, therefore, do not necessarily represent the forms of these chemicals as they now exist. Also not all of these chemicals may have found their way into the SSTs.

Because records are unavailable, quantities of chemicals and the composition of several trade name products could not be determined. Quantities vary from a few pounds to several thousand pounds depending on the operation. Approximately 150 of the chemicals and name products have been identified as hazardous or containing hazardous chemicals, based on substances in the regulations and threshold limit values.

Revision 1 is based on the current level of information about past operations and identification of chemicals that may be regulated. This revision includes addition of ferrocyanide to $T$ Plant and deletion of ferrocyanide from B Plant. 


\subsection{WASTE QUALIFICATION}

The following chemical processes have contributed to the waste stored in the SSTs: the bismuth phosphate process, the uranium recovery (tributyl phosphate) process, the REDOX process, the PUREX process, the Waste Fractionation/Encapsulation Process and the Plutonium Reclamation Process, along with $\mathrm{N}$ Reactor and respective support operations. The chemicals used in these operations were derived from the chemical process flowsheets, essential material consumption records, letters, reports, and other historical data. The other historical data originated in reactor systems and operations groups of the 100 Areas, chemical and fuel reprocessing facilities, tank farms, equipment decontamination, and the analytical laboratories of the 200 Areas, and production support laboratories of the 300 Area.

There is uncertainty about the chemicals used in the laboratory support operations of the 300 Area because not all the identified chemicals were routed to the SSTs. Before 1971, certain wastes from the 300 Area were transported to the 200 Areas for disposal in the cribs or storage in the underground waste tanks. After 1971, radioactive liquid waste was transported to the 200 Area for storage in the underground waste tanks. In addition, certain laboratory waste was mixed or packaged with absorbent materials and then stored as solid waste.

The chemicals listed in this report represent the bulk of nonradioactive chemicals used in facilities that generated and stored radioactive waste in the SSTs since 1944. This information was derived from many sources. The total amounts of chemicals from the laboratory facilities were small compared to the production plants (i.e., 1 pound versus 10,000 pounds). Information on the chemicals used for laboratory support of the early separation plants could not be located.

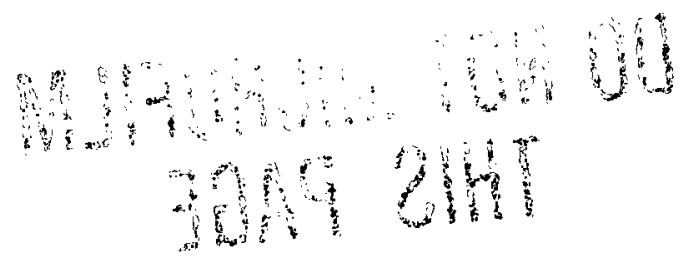


WHC-EP-0172 Revision 1

This page intentionally left blank.

\section{DO NOT MICROFILM THIS PAGE}




\subsection{RESULTS}

Table 1 is the composite list of chemicals used in Hanford Site facilities that possibly discharged waste to the SSTs. The list identifies the chemicals that may be regulated per References 7 and 10 through 14 and is intended for reference purposes. Tables 2 through 35 are individual lists of Hanford Site facilities and chemicals used by these facilities and approximate dates that waste would have been added to the SSTs. Table 36 contains a glossary of product names.

The chemical names shown in the tables are commonly accepted names and are not necessarily those used in rigorous chemical compound nomenclature. 
Table 1. Summary List of Chemicals Used at Hanford Site Production Plants and Support Operations that Stored Waste in the Single-Shell Tanks (1944-1980). (Sheet 1 of 16)

\begin{tabular}{|c|c|c|c|c|c|c|}
\hline Compound name & Formula & RCRA & CERCLA & WAC & OSHA & $\begin{array}{l}\text { OES } \\
\text { TLV }\end{array}$ \\
\hline Acetic Acid & $\mathrm{CH}_{3} \mathrm{CO}_{2} \mathrm{H}$ & & $\mathbf{X}$ & & $\mathbf{X}$ & $\mathbf{X}$ \\
\hline Acetone & $\mathrm{CH}_{3} \mathrm{C}_{2} \mathrm{OH}_{3}$ & $\mathrm{X}$ & $\mathrm{X}$ & $\mathrm{X}$ & $\mathrm{X}$ & $\mathrm{X}$ \\
\hline $\begin{array}{l}\text { Alizarin Yellow } \\
\text { (Dihydroxyanthraquinone) }\end{array}$ & $\mathrm{C}_{14} \mathrm{H}_{8} \mathrm{O}_{4}$ & & & & & \\
\hline Aluminum & $\mathrm{Al}$ & & & & $\mathbf{X}$ & $\mathbf{X}$ \\
\hline Aluminum Nitrate Nonahydrate & $\mathrm{Al}\left(\mathrm{NO}_{3}\right)_{3} \cdot 9 \mathrm{H}_{2} \mathrm{O}$ & & & & $\mathbf{X}$ & $\mathbf{X}$ \\
\hline Aluminum Nitrate (Mono Basic) & $\mathrm{Al}(\mathrm{OH})\left(\mathrm{NO}_{3}\right)_{2}$ & & & & $\mathbf{X}$ & $\mathbf{X}$ \\
\hline Aluminum Nitrate (Di Basic) & $\mathrm{Al}(\mathrm{OH})_{2}\left(\mathrm{NO}_{3}\right)$ & & & & $\mathbf{X}$ & $\mathbf{X}$ \\
\hline Aluminum Sulfate & $\mathrm{Al}_{3}\left(\mathrm{SO}_{4}\right)_{2}$ & & $\mathbf{X}$ & & $\mathbf{X}$ & $\mathbf{X}$ \\
\hline Ammonia (Anhydrous) & $\mathrm{NH}_{3}$ & & $\mathbf{X}$ & & $\mathbf{X}$ & $\mathrm{X}$ \\
\hline Ammonium Acetate & $\mathrm{NH}_{4} \mathrm{C}_{2} \mathrm{H}_{3} \mathrm{O}$ & & $\mathrm{X}$ & & & \\
\hline Ammonium Bisulfate & $\mathrm{NH}_{4} \mathrm{HSO}_{4} \cdot \mathrm{H}_{2} \mathrm{O}$ & & & & & \\
\hline Ammonium Chloride & $\mathrm{NH}_{4} \mathrm{Cl}$ & & $\mathbf{X}$ & & $\mathbf{X}$ & $\mathbf{X}$ \\
\hline Ammonium Fluoride & $\mathrm{NH}_{4} \mathrm{~F}$ & & $\mathbf{X}$ & & $\mathbf{X}$ & $\mathbf{X}$ \\
\hline Ammonium Hydroxide & $\mathrm{NH}_{4} \mathrm{OH}$ & & $\mathbf{X}$ & & & \\
\hline Ammonium Molybdate & $\left(\mathrm{NH}_{4}\right)_{2} \mathrm{MoO}_{4}$ & & & & $\mathrm{X}$ & $\mathrm{x}$ \\
\hline Ammonium Nitrate & $\mathrm{NH}_{4} \mathrm{NO}_{3}$ & & & & & \\
\hline Ammonium Oxalate & $\left(\mathrm{NH}_{4}\right)_{2} \mathrm{C}_{2} \mathrm{O}_{4} \cdot \mathrm{H}_{2} \mathrm{O}$ & & $\mathbf{X}$ & & & \\
\hline Ammonium Fluosilicate & $\left(\mathrm{NH}_{4}\right)_{2} \mathrm{SiF}_{6}$ & & $\mathrm{X}$ & & $\mathrm{X}$ & $\mathbf{X}$ \\
\hline Ammonium Sulfate & $\left(\mathrm{NH}_{4}\right)_{2} \mathrm{SO}_{4}$ & & & & & \\
\hline
\end{tabular}


Table 1. Summary List of Chemicals Used at Hanford Site Production Plants and Support Operations that Stored Waste in the Single-Shell Tanks (1944-1980). (Sheet 2 of 16)

\begin{tabular}{|c|c|c|c|c|c|c|}
\hline Compound name & Formula & RCRA & CERCLA & WAC & OSHA & $\begin{array}{l}\text { OES } \\
\text { TLV }\end{array}$ \\
\hline Ammonium Sulfite & $\left(\mathrm{NH}_{4}\right)_{2} \mathrm{SO}_{3} \cdot \mathrm{H}_{2} \mathrm{O}$ & & $\mathbf{X}$ & & & \\
\hline Ammonium Thiocyanate & $\mathrm{NH}_{4} \mathrm{CNS}$ & & $\mathrm{X}$ & & & \\
\hline Ammonium Thiosulfate & $\left(\mathrm{NH}_{4}\right)_{2} \mathrm{~S}_{2} \mathrm{O}_{3}$ & & $\mathbf{X}$ & & & \\
\hline Antimony Chloride & $\mathrm{Sb}(\mathrm{Cl})_{3}$ & $\mathbf{X}$ & $\mathrm{X}$ & & $\mathbf{X}$ & $\mathrm{X}$ \\
\hline Antimony Nitrate & $\mathrm{Sb}\left(\mathrm{NO}_{3}\right)_{3}$ & $\mathbf{X}$ & $\mathbf{X}$ & & $\mathrm{X}$ & $\mathrm{X}$ \\
\hline Arsenic Trioxide & $\mathrm{As}_{2} \mathrm{O}_{3}$ & $\mathbf{X}$ & $\mathbf{X}$ & $\mathrm{X}$ & & $\mathbf{X}$ \\
\hline Barium Nitrate & $\mathrm{Ba}\left(\mathrm{NO}_{3}\right)_{2}$ & $\mathbf{x}$ & $\mathrm{X}$ & $\mathbf{X}$ & $\mathbf{X}$ & $\mathrm{X}$ \\
\hline Benzene & $\mathrm{C}_{6} \mathrm{H}_{6}$ & $\mathbf{x}$ & $\mathrm{X}$ & $x$ & $\mathrm{X}$ & $\mathbf{X}$ \\
\hline Beryllium & $\mathrm{Be}$ & $\mathbf{X}$ & $\mathbf{X}$ & $\mathbf{X}$ & $\mathbf{X}$ & $\mathbf{X}$ \\
\hline Boric Acid & $\mathrm{H}_{3} \mathrm{BO}_{3}$ & . & & & & \\
\hline Boron Carbide & $\mathrm{B}_{4} \mathrm{C}$ & & & & & \\
\hline Bromine & $\mathrm{Br}_{2}$ & & & & $\mathbf{X}$ & $\mathbf{X}$ \\
\hline Bromocresol Purple & $\mathrm{C}_{7} \mathrm{H}_{6} \mathrm{OHBr}$ & $\mathbf{X}$ & $\mathbf{X}$ & $\mathbf{X}$ & $\mathrm{X}$ & $\mathbf{X}$ \\
\hline Bromonaphthalene & $\mathrm{C}_{10} \mathrm{H}_{7} \mathrm{Br}$ & & & & & \\
\hline Butanol & $\mathrm{C}_{4} \mathrm{H}_{9} \mathrm{OH}$ & $\mathrm{X}$ & $\mathrm{X}$ & $\mathrm{X}$ & $\mathbf{X}$ & $\mathbf{X}$ \\
\hline Cadmium Nitrate & $\mathrm{Cd}\left(\mathrm{NO}_{3}\right)_{2}$ & $\mathrm{X}$ & $\mathbf{X}$ & $\mathrm{X}$ & & $\mathbf{X}$ \\
\hline Calcium & $\mathrm{Ca}$ & & & & & \\
\hline Calcium Carbonate & $\mathrm{CaCO}_{3}$ & & & & $\mathbf{X}$ & $\mathbf{X}$ \\
\hline Calcium Chloride & $\mathrm{CaCl}_{2}$ & & & & & \\
\hline Calcium Nitrate & $\mathrm{Ca}\left(\mathrm{NO}_{3}\right)_{2}$ & & & & & \\
\hline
\end{tabular}


Table 1. Summary List of Chemicals Used at Hanford Site Production Plants and Support Operations that Stored Waste in the Single-Shell Tanks (1944-1980). (Sheet 3 of 16)

\begin{tabular}{|c|c|c|c|c|c|c|}
\hline Compound name & Formula & RCRA & CERCLA & WAC & OSHA & $\begin{array}{l}\text { OES } \\
\text { TLV }\end{array}$ \\
\hline Carbon Dioxide & $\mathrm{CO}_{2}$ & & & & $\mathbf{X}$ & $\mathbf{X}$ \\
\hline Carbon Tetrachloride & $\mathrm{CCl}_{4}$ & $\mathrm{X}$ & $\mathrm{X}$ & $\mathbf{X}$ & $\mathbf{X}$ & $\mathbf{X}$ \\
\hline Ceric Ammonium Nitrate & $\mathrm{Ce}\left(\mathrm{NH}_{4}\right)_{2}\left(\mathrm{NO}_{3}\right)_{6}$ & & & & & \\
\hline Ceric Fluoride & $\mathrm{CeF}_{4}$ & & & & $\mathbf{X}$ & $\mathbf{X}$ \\
\hline Ceric Iodate & $\mathrm{Ce}\left(\mathrm{IO}_{3}\right)_{4}$ & & & & & \\
\hline Ceric Nitrate & $\mathrm{Ce}\left(\mathrm{NO}_{3}\right)_{4}$ & & & & & \\
\hline Ceric Sulfate & $\mathrm{Ce}\left(\mathrm{SO}_{4}\right)_{2}$ & & & & & \\
\hline Cerous Nitrate & $\mathrm{Ce}\left(\mathrm{NO}_{3}\right)_{3}$ & & & & & \\
\hline Cesium & Cs & & & & & \\
\hline Cesium Chloride & $\mathrm{CsCl}$ & & & & & \\
\hline Cesium Nitrate & $\mathrm{CsNO}_{3}$ & & & & & \\
\hline Cesium Nitrite & $\mathrm{CsNO}_{2}$ & & & & & \\
\hline Chloroplatinic Acid & $\mathrm{H}_{2} \mathrm{PtCl}_{6} \cdot 6 \mathrm{H}_{2} \mathrm{O}$ & & & & $\mathbf{X}$ & $\mathbf{X}$ \\
\hline Chromic Acid & $\mathrm{H}_{2} \mathrm{CrO}_{4}$ & $\mathrm{X}$ & $\mathrm{x}$ & $\mathrm{X}$ & $\mathrm{X}$ & $\mathrm{X}$ \\
\hline Chromium Nitrate & $\mathrm{Cr}\left(\mathrm{NO}_{3}\right)_{3}$ & $\mathrm{X}$ & $\mathbf{X}$ & $\mathrm{X}$ & $\mathbf{X}$ & $\mathbf{X}$ \\
\hline Chromous Sulfate & $\mathrm{CrSO}_{4} \cdot 7 \mathrm{H}_{2} \mathrm{O}$ & $\mathrm{X}$ & $\mathrm{X}$ & $\mathrm{X}$ & $\mathbf{X}$ & $\bar{X}$ \\
\hline Citric Acid & $\mathrm{C}_{3} \mathrm{H}_{4} \mathrm{OH}(\mathrm{COOH})_{3}$ & & & & & \\
\hline Cobalt Sulfate & $\mathrm{CoSO}_{4} \cdot 7 \mathrm{H}_{2} \mathrm{O}$ & & & & & \\
\hline Copper & $\mathrm{Cu}$ & & $\mathbf{X}$ & & $\mathbf{X}$ & $\mathbf{X}$ \\
\hline Copper Nitrate & $\mathrm{Cu}\left(\mathrm{NO}_{3}\right)_{2}$ & & $\bar{X}$ & & & \\
\hline Cupric Sulfate & $\mathrm{CuSO}_{4} \cdot 5 \mathrm{H}_{2} \mathrm{O}$ & & $\mathrm{X}$ & & & \\
\hline
\end{tabular}


Table 1. Summary List of Chemicals Used at Hanford Site Production Plants and Support Operations that Stored Waste in the Single-Shell Tanks (1944-1980). (Sheet 4 of 16)

\begin{tabular}{|c|c|c|c|c|c|c|}
\hline Compound rame & Formula & RCRA & CERCLA & WAC & OSHA & $\begin{array}{l}\text { OES } \\
\text { TLV }\end{array}$ \\
\hline Diatomateous Earth & $\mathrm{SiO}_{2}$ & & & & $\mathbf{X}$ & $\mathbf{X}$ \\
\hline Di2-Ethyl Hexyl Phosphoric Acid & $\mathrm{C}_{16} \mathrm{H}_{34} \mathrm{O}_{2} \mathrm{POOH}$ & & & & & \\
\hline Dibutyl Butyl Phosphonate & $\mathrm{CH}_{3}\left(\mathrm{CH}_{2}\right)_{3} \mathrm{PO}_{3}\left[\mathrm{CH}_{3}\left(\mathrm{CH}_{2}\right)_{3}\right]_{2}$ & & & & & \\
\hline Dibutyl Phosphate & $\left(\mathrm{n}-\mathrm{C}_{4} \mathrm{H}_{9}\right)_{2} \mathrm{HPO}_{4}$ & & & & $\mathrm{X}$ & $\mathbf{X}$ \\
\hline Dodecane & $\mathrm{CH}_{3}\left(\mathrm{CH}_{2}\right)_{10} \mathrm{CH}_{3}$ & & & & & \\
\hline Ethanol & $\mathrm{C}_{2} \mathrm{H}_{5} \mathrm{OH}$ & & & & $\mathbf{X}$ & $\mathbf{X}$ \\
\hline Ethyl Ether & $\left(\mathrm{CH}_{3} \mathrm{CH}_{2}\right)_{2} \mathrm{O}$ & $\mathbf{X}$ & $\mathbf{X}$ & $\mathbf{X}$ & $\mathrm{X}$ & $\mathrm{X}$ \\
\hline Ethylene Glycol & $\mathrm{HOCH}_{2} \mathrm{CH}_{2} \mathrm{OH}$ & & & & $\mathbf{X}$ & $\mathbf{X}$ \\
\hline Perric Ammonium Sulfate & $\mathrm{FeNH}_{4}\left(\mathrm{SO}_{4}\right)_{2}$ & & & & $\mathrm{X}$ & $\mathbf{x}$ \\
\hline Ferric Nitrate & $\mathrm{Fe}\left(\mathrm{NO}_{3}\right)_{3} \cdot 6 \mathrm{H}_{2} \mathrm{O}$ & & $\mathbf{X}$ & & $\mathbf{X}$ & $\mathbf{X}$ \\
\hline Perric Sulfate & $\mathrm{Fe}_{2}\left(\mathrm{SO}_{4}\right)_{3}$ & & $\mathbf{X}$ & & $\mathrm{X}$ & $\mathbf{X}$ \\
\hline Ferrous Ammonium Sulfate & $\mathrm{Pe}\left(\mathrm{NH}_{4}\right)_{2}\left(\mathrm{SO}_{4}\right)_{2} \cdot 6 \mathrm{H}_{2} \mathrm{O}$ & & $\mathbf{X}$ & & $\mathrm{X}$ & $\mathbf{X}$ \\
\hline Ferrous Sulfamate & $\mathrm{Fe}\left(\mathrm{SO}_{3} \mathrm{NH}_{2}\right)_{2}$ & & & & $\mathbf{X}$ & $\mathbf{X}$ \\
\hline Ferrous Sulfate & $\mathrm{FeSO}_{4}$ & & $\mathrm{X}$ & & $\mathbf{X}$ & $\mathbf{X}$ \\
\hline Formaldehyde (Soluticn) & $\mathrm{CH}_{2} \mathrm{O}$ & $\mathrm{X}$ & $\mathrm{X}$ & $\mathrm{X}$ & $\mathrm{X}$ & $\mathrm{X}$ \\
\hline Gallium & $\mathrm{Ga}$ & & & & & \\
\hline Gallium Oxide & $\mathrm{Ga}_{2} \mathrm{O}_{3}$ & & & & & \\
\hline Glycolic Acid & $\mathrm{HOCH}_{2} \mathrm{CO}_{2} \mathrm{H}$ & & & & & \\
\hline Glycerol & $\mathrm{CH}_{2} \mathrm{OHCHOHCH}{ }_{2} \mathrm{OH}$ & & & & & \\
\hline Gold & $\mathrm{Au}$ & & & & & \\
\hline
\end{tabular}


Table 1. Summary List of Chemicals Used at Hanford Site Production Plants and Support Operations that Stored Waste in the Single-Shell Tanks (1944-1980). (Sheet 5 of 16)

\begin{tabular}{|c|c|c|c|c|c|c|}
\hline Compound name & Formula & RCRA & CERCLA & WAC & OSHA & $\begin{array}{l}\text { OES } \\
\text { TLV }\end{array}$ \\
\hline Hydrazine & $\mathrm{H}_{2} \mathrm{NNH}_{2} \cdot \mathrm{H}_{2} \mathrm{O}$ & $\mathbf{X}$ & $\mathbf{X}$ & $\mathbf{X}$ & $\mathbf{X}$ & $\mathbf{X}$ \\
\hline Hydrobromic Acid & $\mathrm{HBr}$ & & & & $\mathbf{X}$ & $\mathbf{X}$ \\
\hline Hydrochloric Acid & $\mathrm{HCl}$ & & $\mathbf{X}$ & & $\mathbf{X}$ & $\mathbf{X}$ \\
\hline Hydrofluoric Acid & HF & $\mathbf{X}$ & $\mathbf{X}$ & $\mathrm{X}$ & $\mathbf{X}$ & $\mathbf{X}$ \\
\hline Hydrogen Peroxide & $\mathrm{H}_{2} \mathrm{O}_{2}$ & & & & $\mathbf{X}$ & $\mathbf{X}$ \\
\hline Hydrogen Sulfide & $\mathrm{H}_{2} \mathrm{~S}$ & $\mathbf{X}$ & $\mathbf{X}$ & $\mathbf{X}$ & $\mathbf{X}$ & $\mathrm{X}$ \\
\hline Hydroiodic Acid & HI & & & & & \\
\hline Hydroxyacetic Acid & $\mathrm{CH}_{3} \mathrm{OCOOH}$ & & & & & \\
\hline Hydroxylamine Hydrochloride & $\mathrm{NH}_{2} \mathrm{OH} \cdot \mathrm{HCl}$ & & & & & \\
\hline Hydroxylamine Nitrate & $\mathrm{NH}_{2} \mathrm{OH} \cdot \mathrm{HNO}_{3}$ & & & & & \\
\hline Hydroxyquinoline & $\mathrm{C}_{9} \mathrm{H}_{6} \mathrm{NOH}$ & & & & & \\
\hline Iodine & $I_{2}$ & & & & $\mathbf{x}$ & $\mathbf{x}$ \\
\hline Iron & $\mathrm{Fe}$ & & & & & \\
\hline Isoamyl Acetate & $\mathrm{C}_{7} \mathrm{H}_{14} \mathrm{O}_{2}$ & & & & $\mathbf{X}$ & $\bar{X}$ \\
\hline Isopropyl Alcohol & $\mathrm{C}_{3} \mathrm{H}_{7} \mathrm{OH}$ & & & & $\bar{x}$ & $\mathrm{X}$ \\
\hline Kerosene (Inhibited) & $\mathrm{C}_{10} \mathrm{H}_{22}$ to $\mathrm{C}_{16} \mathrm{H}_{34}+\mathrm{Cl}_{2} \mathrm{CCHCl}$ & $\mathrm{X}$ & $\mathrm{X}$ & $\mathrm{X}$ & $\mathrm{X}$ & $\bar{X}$ \\
\hline Kerosene & $\mathrm{C}_{10} \mathrm{H}_{22}$ to $\mathrm{C}_{16} \mathrm{H}_{34}$ & & & & & \\
\hline Lanthanum Chloride & $\mathrm{LaCl}_{3}$ & & & & & 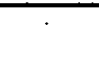 \\
\hline Lanthanum Fluoride & $\mathrm{LaF}_{3}$ & & & & $\bar{x}$ & $\mathrm{x}$ \\
\hline Lanthanum Hydroxide & $\mathrm{La}(\mathrm{OH})_{3}$ & & & & & \\
\hline
\end{tabular}


Table 1. Summary List of Chemicals Used at Hanford Site Production Plants and Support Operations that Stored Waste in the Single-Shell Tanks (1944-1980). (Sheet 6 of 16)

\begin{tabular}{|c|c|c|c|c|c|c|}
\hline Compound name & Formula & RCRA & CERCLA & WAC & OSHA & $\begin{array}{l}\text { OES } \\
\text { TLV }\end{array}$ \\
\hline Lanthanum-Neodønium Nitrate & $\mathrm{La}\left(\mathrm{NO}_{3}\right)_{3}-\mathrm{Nd}\left(\mathrm{NO}_{3}\right)_{2}$ & & & & & \\
\hline Lanthanum Nitrate & $\mathrm{La}\left(\mathrm{NO}_{3}\right)_{3}$ & & & & & \\
\hline Lead Nitrate & $\mathrm{Pb}\left(\mathrm{NO}_{3}\right)_{2}$ & $\mathbf{X}$ & $\mathrm{X}$ & $\mathbf{X}$ & & \\
\hline Lithium & $\mathrm{Li}$ & & & & & \\
\hline Magnesium & $\mathrm{Mg}$ & & & & & \\
\hline Magnesium Carbonate & $\mathrm{MgCO}_{3}$ & & & & & \\
\hline Magnesium Nitrate & $\mathrm{Mg}\left(\mathrm{NO}_{3}\right)_{2}$ & & & & & \\
\hline Magnesium Oxide & $\mathrm{MgO}$ & & & & $\mathbf{X}$ & $\mathbf{X}$ \\
\hline Mandelic Acid & $\mathrm{C}_{6} \mathrm{H}_{5} \mathrm{CHOHCO}{ }_{2} \mathrm{H}$ & & & & & \\
\hline Mercury & $\mathrm{Hg}$ & $\mathbf{X}$ & $\mathbf{X}$ & $\mathbf{X}$ & $\mathbf{X}$ & $\mathbf{X}$ \\
\hline Mercuric Nitrate & $\mathrm{Hg}\left(\mathrm{NO}_{3}\right)_{2} \cdot \mathrm{H}_{2} \mathrm{O}$ & $\mathrm{X}$ & $\mathrm{X}$ & $\mathrm{X}$ & $\mathbf{X}$ & $\mathrm{X}$ \\
\hline Mercuric Thiocyanate & $\mathrm{Hg}(\mathrm{SCN})_{2}$ & $\mathbf{X}$ & $\mathbf{X}$ & $\mathbf{X}$ & $\mathrm{X}$ & $\mathbf{X}$ \\
\hline Methanol & $\mathrm{CH}_{3} \mathrm{OH}$ & $\mathrm{X}$ & $\mathrm{X}$ & $\mathrm{X}$ & $\mathbf{X}$ & $\mathbf{X}$ \\
\hline Methyl Ethyl Ketcne & $\mathrm{CH}_{3} \mathrm{COC}_{2} \mathrm{H}_{5}$ & $\mathrm{X}$ & $\mathbf{X}$ & $\mathrm{X}$ & $\mathbf{X}$ & $\mathrm{X}$ \\
\hline Methyl Isobutyl Ketone & $\mathrm{CH}_{3} \mathrm{COC}_{4} \mathrm{H}_{9}$ & $\mathbf{X}$ & $\mathrm{X}$ & $\mathbf{X}$ & $\mathbf{X}$ & $\mathrm{X}$ \\
\hline Methyl Lactic Acid & $\mathrm{CH}_{3} \mathrm{CH}(\mathrm{OH}) \mathrm{CO}_{2} \mathrm{CH}_{3}$ & & & & & \\
\hline Molybdate Citrate, Reagent & $\mathrm{MoO}_{3} \cdot \mathrm{XH}_{2} \mathrm{O}+\left(\mathrm{NH}_{4}\right)_{3} \mathrm{C}_{6} \mathrm{H}_{5} \mathrm{O}_{7}$ & & & & $\mathbf{X}$ & $\mathbf{X}$ \\
\hline Molybdenum & Mo & & & . & & \\
\hline Morpholine & $\mathrm{C}_{4} \mathrm{H}_{9} \mathrm{NO}$ & & & & $\mathrm{X}$ & $\mathbf{X}$ \\
\hline Naphthylamine & $\mathrm{C}_{10} \mathrm{H}_{9} \mathrm{~N}$ & $\mathrm{X}$ & $\mathbf{X}$ & $\mathbf{X}$ & & $\mathrm{x}$ \\
\hline Neodymium Chloride & $\mathrm{NdCl}_{3}$ & & & & & \\
\hline
\end{tabular}


Table 1. Summary List of Chemicals Used at Hanford Site Production Plants and Support Operations that Stored Waste in the Single-Shell Tanks (1944-1980). (Sheet 7 of 16)

\begin{tabular}{|c|c|c|c|c|c|c|}
\hline Compound name & Formula & RCRA & CERCLA & WAC & OSHA & OES \\
\hline Neodymium Nitrate & $\mathrm{Nd}\left(\mathrm{NO}_{3}\right)_{3}$ & & & & & \\
\hline Nickel & $\mathrm{Ni}$ & $\mathbf{X}$ & $\mathbf{X}$ & $\mathbf{X}$ & $\mathbf{X}$ & $\mathbf{X}$ \\
\hline Nickel Nitrate & $\mathrm{Ni}\left(\mathrm{NO}_{3}\right)_{2} \cdot 6 \mathrm{H}_{2} \mathrm{O}$ & $\mathbf{X}$ & $\mathbf{X}$ & $\mathbf{X}$ & $\mathbf{X}$ & $\mathrm{X}$ \\
\hline Nickel Sulfate & $\mathrm{NiSO}_{4}$ & $\mathbf{X}$ & $\mathrm{X}$ & $\mathrm{X}$ & $\mathbf{X}$ & $\mathrm{X}$ \\
\hline Niobium & $\mathrm{Nb}$ & & & & & \\
\hline Nitric Acid & $\mathrm{HNO}_{3}$ & & $\mathrm{X}$ & & $\mathrm{X}$ & $\mathbf{X}$ \\
\hline Nitrilotriacetic Acid (NTA) & $\mathrm{N}\left(\mathrm{CH}_{2} \mathrm{COOH}\right)_{3}$ & & & & & \\
\hline Nitrous Acid & $\mathrm{HNO}_{2}$ & & & & & \\
\hline Normal Paraffin Hydrocarbon & $\mathrm{C}_{10} \mathrm{H}_{22}$ to $\mathrm{C}_{14} \mathrm{H}_{30}$ & & & & & \\
\hline O-phenanthroline & $\mathrm{C}_{12} \mathrm{H}_{8} \mathrm{~N}_{2}$ & & & & & \\
\hline Oxalic Acid & $\mathrm{HO}_{2} \mathrm{CCO}_{2} \mathrm{H} \cdot 2 \mathrm{H}_{2} \mathrm{O}$ & & & & $\mathrm{X}$ & $\mathrm{X}$ \\
\hline $\begin{array}{l}\text { Pentasodium Diethylene-Triamine } \\
\text { Penta Acetate (DTPA) }\end{array}$ & $\mathrm{N}_{3}\left(\mathrm{C}_{2} \mathrm{H}_{2}\right)_{2}(\mathrm{COONa})_{5}$ & & & & & \\
\hline Perchloric Acid & $\mathrm{HClO}_{4}$ & & & & & \\
\hline Periodic Acid & $\mathrm{HIO}_{4}$ & & & & & \\
\hline Phosphoric Acid & $\mathrm{H}_{3} \mathrm{PO}_{4}$ & & $\mathbf{X}$ & & $\mathbf{X}$ & $\mathbf{X}$ \\
\hline Phosphorous Pentoxide & $\mathrm{P}_{2} \mathrm{O}_{5}$ & & & & & \\
\hline Phosphotungstic Acid & $\mathrm{H}_{3}\left[\mathrm{P}\left(\mathrm{W}_{3} \mathrm{O}_{10}\right)_{4}\right] \cdot 14 \mathrm{H}_{2} \mathrm{O}$ & & & & $\mathrm{X}$ & $\mathrm{X}$ \\
\hline Platinum & $\mathrm{Pt}$ & & & & $\mathbf{X}$ & $\mathbf{X}$ \\
\hline
\end{tabular}


Table 1. Summary List of Chemicals Used at Hanford Site Production Plants and Support Operations that Stored Waste in the Sirgle-Shell Tanks (1944-1980). (Sheet 8 of 16)

\begin{tabular}{|c|c|c|c|c|c|c|}
\hline Compound name & Formula & RCRA & CERCLA & WAC & OSHA & $\begin{array}{l}\text { OES } \\
\text { TLV }\end{array}$ \\
\hline Potassium Acetate & $\mathrm{KC}_{2} \mathrm{H}_{3} \mathrm{O}_{2}$ & & & & & \\
\hline Potassium Bicarbonate & $\mathrm{KHCO}_{3}$ & & & & & \\
\hline Potassium Bisulfite & $\mathrm{K}_{2} \mathrm{~S}_{2} \mathrm{O}_{5}$ & & & & & \\
\hline Potassium Carbonate & $\mathrm{K}_{2} \mathrm{CO}_{3}$ & & & & & \\
\hline Potassium Cyanide & $\mathrm{KCN}$ & $\mathrm{X}$ & $\mathbf{X}$ & $\mathbf{X}$ & $\mathbf{X}$ & $\mathbf{X}$ \\
\hline Potassium Dichromate & $\mathrm{K}_{2} \mathrm{CrO}_{7}$ & $\mathbf{X}$ & $\mathbf{X}$ & $\mathbf{X}$ & $\mathbf{X}$ & \\
\hline Patassium Ferricyanide & $\mathrm{K}_{3} \mathrm{Fe}(\mathrm{CN})_{6}$ & $\mathrm{X}$ & $\mathrm{X}$ & $\mathbf{X}$ & $\mathbf{X}$ & $\mathbf{X}$ \\
\hline Potassium Ferrocyanide & $\mathrm{K}_{4} \mathrm{Fe}(\mathrm{CN})_{6}$ & $\mathbf{X}$ & $\mathrm{X}$ & $\mathrm{X}$ & $\mathbf{X}$ & $\mathbf{X}$ \\
\hline Pctassium Fluoride & $\mathbf{K F}$ & & & & $\mathbf{X}$ & $\mathbf{X}$ \\
\hline Pctassium Hydroxide & $\mathrm{KOH}$ & & $\mathbf{X}$ & & $\mathbf{X}$ & $\mathbf{X}$ \\
\hline Pctassium Iodate & $\mathrm{KiO}_{3}$ & & & & & \\
\hline Pctassium Oxalate & $\mathrm{K}_{2} \mathrm{C}_{2} \mathrm{O}_{4}$ & & & & & \\
\hline Potassium Oxide & $\mathrm{K}_{2} \mathrm{O}$ & & & & & \\
\hline Potassium Permanganate & $\mathrm{KMnO}_{4}$ & & $\mathbf{X}$ & & & \\
\hline Potassium Persulfate & $\mathrm{K}_{2} \mathrm{~S}_{2} \mathrm{O}_{8}$ & & & & & \\
\hline Praseodymium Chloride & $\mathrm{PrCl}_{2}$ & & & & & \\
\hline Praseodymium Nitrate & $\operatorname{Pr}\left(\mathrm{NO}_{3}\right)_{2}$ & & & & & \\
\hline S-diphenyl Carbazide & $\left(\mathrm{C}_{6} \mathrm{H}_{5} \mathrm{NH} \cdot \mathrm{HN}\right)_{2} \mathrm{CO}$ & & & & & \\
\hline Selenium Chloride & $\mathrm{SeCl}_{4}$ & $\mathbf{X}$ & $\mathbf{X}$ & $\mathrm{X}$ & $\mathbf{X}$ & $\mathbf{X}$ \\
\hline Selenium Nitrate & $\mathrm{Se}\left(\mathrm{NO}_{3}\right)_{4}$ & $\mathrm{X}$ & $\mathrm{X}$ & $\mathbf{X}$ & $\mathbf{X}$ & $\mathbf{X}$ \\
\hline
\end{tabular}


Table 1. Summary List of Chemicals Used at Hanford Site Production Plants and Support Operations that Stored Waste in the Single-Shell Tanks (1944-1980). (Sheet 9 of 16)

\begin{tabular}{|c|c|c|c|c|c|c|}
\hline Compound name & Formula & RCRA & CERCLA & WAC & OSHA & $\begin{array}{l}\text { OES } \\
\text { TLV }\end{array}$ \\
\hline Silicon & $\mathrm{Si}$ & & & & $\mathbf{X}$ & $\mathbf{X}$ \\
\hline Silico Tungstic Acid & $\mathrm{H}_{4}\left[\mathrm{Si}\left(\mathrm{W}_{3} \mathrm{O}_{10}\right)_{4}\right] \cdot 26 \mathrm{H}_{2} \mathrm{O}$ & & & & $\mathbf{X}$ & $\mathbf{X}$ \\
\hline Silver & $\mathrm{Ag}$ & $\mathbf{X}$ & $\mathbf{X}$ & $\mathbf{X}$ & $\mathbf{X}$ & $\mathbf{X}$ \\
\hline Silver Chloride & $\mathrm{AgCl}$ & $\mathbf{X}$ & $\mathbf{x}$ & $\mathbf{X}$ & $\mathbf{X}$ & $\mathbf{X}$ \\
\hline Silver Nitrate & $\mathrm{AgNO}_{3}$ & $\mathrm{X}$ & $\mathbf{X}$ & $\mathrm{X}$ & $\mathrm{X}$ & $\mathrm{X}$ \\
\hline Silver Oxide & $\mathrm{AgO}$ & $\mathrm{X}$ & $\mathbf{X}$ & $\mathrm{X}$ & $\mathbf{X}$ & $\mathbf{X}$ \\
\hline Sodium & $\mathrm{Na}$ & & $\mathbf{X}$ & & & \\
\hline Sodium Acetate & $\mathrm{NaC}_{2} \mathrm{H}_{3} \mathrm{O}_{2}$ & & & & & \\
\hline Sodium Bicarbonate & $\mathrm{NaHCO}_{3}$ & & & & & \\
\hline Sodium Bismuthate & $\mathrm{NaBiO}_{3}$ & & & & & \\
\hline Sodium Bisulfate & $\mathrm{NaHSO}_{4} \cdot \mathrm{H}_{2} \mathrm{O}$ & & & & & \\
\hline Sodium Bromate & $\mathrm{NaBrO}_{3}$ & & & & & \\
\hline Sodium Carbonate & $\mathrm{Na}_{2} \mathrm{CO}_{3}$ & & & & & \\
\hline Sodium Dichromate & $\mathrm{Na}_{2} \mathrm{Cr}_{2} \mathrm{O}_{7} \cdot 2 \mathrm{H}_{2} \mathrm{O}$ & $\mathbf{x}$ & $\mathbf{X}$ & $\mathbf{x}$ & $\mathbf{X}$ & $\mathbf{X}$ \\
\hline Sodium Ferrocyanide & $\mathrm{Na}_{4} \mathrm{Fe}(\mathrm{CN})_{6}$ & $\mathbf{x}$ & $\mathbf{X}$ & $\mathbf{X}$ & $\mathrm{X}$ & $\mathrm{X}$ \\
\hline Sodium Fluoride & $\mathrm{NaF}$ & & $\mathbf{X}$ & & $\mathrm{X}$ & $\mathbf{X}$ \\
\hline Sodium Gluconate & $(\mathrm{CHOH})_{4} \mathrm{CO}_{2} \mathrm{HCH}_{2} \mathrm{ONa}$ & & & & & \\
\hline Sodium Hydroxide & $\mathrm{NaOH}$ & & $\mathbf{X}$ & & $\mathbf{X}$ & $\mathbf{X}$ \\
\hline Sodium Hypochlorite & $\mathrm{NaOCl}$ & & $\mathrm{X}$ & & & \\
\hline Sodium Iodide & $\mathrm{NaI}$ & & $\bar{s}$ & & & \\
\hline
\end{tabular}


Table 1. Summary List of Chemicals Used at Hanford Site Production Plants and Support Operations that Stored Waste in the Single-Shell Tanks (1944-1980). (Sheet 10 of 16)

\begin{tabular}{|c|c|c|c|c|c|c|}
\hline Compound name & Formula & RCRA & CERCLA & WAC & OSHA & $\begin{array}{l}\text { OES } \\
\text { TLV }\end{array}$ \\
\hline Sodium Nitrate & $\mathrm{NaNO}_{3}$ & & & 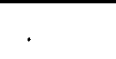 & & \\
\hline Sadium Nitrite & $\mathrm{NaNO}_{2}$ & & $\mathrm{X}$ & & & \\
\hline Sodium Oxalate & $\mathrm{Na}_{2} \mathrm{C}_{2} \mathrm{O}_{4}$ & & & & & \\
\hline Sodium Persulfate & $\mathrm{Na}_{2} \mathrm{SO}_{2} \mathrm{O}_{8}$ & & & & & \\
\hline Sodium Phosphate & $\mathrm{Na}_{3} \mathrm{PO}_{4}$ & & $\mathbf{X}$ & & & \\
\hline Sodium Silicate & $\mathrm{Na}_{2} \mathrm{O} \cdot \mathrm{SiO}_{2}$ & & & & & \\
\hline Sodium Sulfate & $\mathrm{Na}_{2} \mathrm{SO}_{4}$ & & & & & \\
\hline Sodium Sulfide & $\mathrm{Na}_{2} \mathrm{~S}$ & & & & & \\
\hline Sodium Tartrate & $\mathrm{Na}_{2} \mathrm{C}_{2} \mathrm{H}_{4} \mathrm{O}_{6} \cdot 2 \mathrm{H}_{2} \mathrm{O}$ & & & & & \\
\hline Sodium Thiocyanate & $\mathrm{NaSCN}$ & & & & & \\
\hline Sodium Thiosulfate & $\mathrm{Na}_{2} \mathrm{~S}_{2} \mathrm{O}_{3}$ & & & & & \\
\hline Stainless Steel & $\mathrm{FeNiCr}$ & $\mathrm{X}$ & $\mathbf{X}$ & $\mathbf{X}$ & $\mathbf{x}$ & $\mathbf{X}$ \\
\hline Strontium Chloride & $\mathrm{SrCl}_{2}$ & & & & & \\
\hline Strontium Fluoride & $\mathrm{SrF}_{2}$ & & & & $\mathbf{X}$ & $\mathbf{X}$ \\
\hline Strontium Nitrate & $\mathrm{Sr}\left(\mathrm{NO}_{3}\right)_{2}$ & & & & & \\
\hline Sugar & $\mathrm{C}_{12} \mathrm{H}_{22} \mathrm{O}_{11}$ & & & & $\mathbf{X}$ & $\mathbf{X}$ \\
\hline Sulfamic Acid & $\mathrm{NH}_{2} \mathrm{SO}_{3} \mathrm{H}$ & & & & & \\
\hline Sulfonic Acid (chloro) & $\mathrm{ClHSO}_{3}$ & & $\mathrm{X}$ & & & \\
\hline Sulfuric Acid & $\mathrm{H}_{2} \mathrm{SO}_{4}$ & & $\mathrm{x}$ & & $\mathbf{X}$ & $\mathbf{X}$ \\
\hline Tartaric Acid & $\mathrm{C}_{2} \mathrm{H}_{2}(\mathrm{OHCOOH})_{2}$ & & & & & \\
\hline
\end{tabular}


Table 1. Summary List of Chemicals Used at Hanford Site Production Plants and Support Operations that Stored Waste in the Single-Shell Tanks (1944-1980). (Sheet 11 of 16)

\begin{tabular}{|c|c|c|c|c|c|c|}
\hline Compound name & Formula & RCRA & CERCLA & WAC & OSHA & $\begin{array}{l}\text { OES } \\
\text { TLV }\end{array}$ \\
\hline Tantalum & $\mathrm{Ta}$ & & & & $\mathbf{X}$ & $\mathrm{X}$ \\
\hline $\begin{array}{l}\text { Tetrasodium Ethylene Diamine- } \\
\text { Tetra Acetate (ED'TA) }\end{array}$ & $\mathrm{N}_{2} \mathrm{C}_{2} \mathrm{H}_{4}\left(\mathrm{C}_{2} \mathrm{H}_{2} \mathrm{O}_{2} \mathrm{Na}\right)_{4}$ & & $\mathbf{X}$ & & & \\
\hline Tetrabromoethane & $\left(\mathrm{CHBr}_{2}\right)_{2}$ & & & & & \\
\hline Tetraphenyl Boron & $\left(\mathrm{C}_{6} \mathrm{H}_{5}\right)_{4} \mathrm{~B}$ & & & & & \\
\hline Thenoyltrifluoroacetone & $(\mathrm{CH})_{3} \mathrm{SCOCH}_{2} \mathrm{COCF}_{3}$ & & & & & \\
\hline Thorium Chloride & $\mathrm{ThCl}_{4}$ & & & & & \\
\hline Thorium Nitrate & $\mathrm{Th}\left(\mathrm{NO}_{3}\right)_{4}$ & & & & & \\
\hline Thymolpthalein & $\mathrm{C}_{28} \mathrm{H}_{30} \mathrm{O}_{4}$ & & & & & \\
\hline Tin Chloride & $\mathrm{SnCl}_{4}$ & & & & $\mathbf{X}$ & $\mathbf{X}$ \\
\hline Tin Nitrate & $\mathrm{Sn}\left(\mathrm{NO}_{3}\right)_{4}$ & & & & $\mathbf{X}$ & $\mathbf{X}$ \\
\hline Titanium Chloride & $\mathrm{TiCl}_{4}$ & & & & & \\
\hline Toluene & $\mathrm{C}_{6} \mathrm{H}_{5} \mathrm{CH}_{3}$ & $\mathbf{X}$ & $\mathrm{X}$ & $\mathbf{X}$ & $\mathbf{X}$ & $\mathbf{X}$ \\
\hline Tributyl Phosphate & $\left(\mathrm{C}_{4} \mathrm{H}_{9}\right)_{3} \mathrm{PO}_{4}$ & & & & $\mathbf{X}$ & $\mathbf{X}$ \\
\hline Trichloroethane & $\mathrm{CH}_{3} \mathrm{CCl}_{3}$ & $\mathrm{X}$ & $\mathrm{X}$ & $\mathrm{X}$ & $\mathbf{X}$ & $\mathrm{X}$ \\
\hline Tri-n-dodecylamine & {$\left[\mathrm{CH}_{3}\left(\mathrm{CH}_{2}\right)_{11}\right]_{3} \mathrm{~N}$} & & & & & \\
\hline Tri-Iso-octylamine & {$\left[\left(\mathrm{CH}_{3}\right)_{2} \mathrm{CH}\left(\mathrm{CH}_{2}\right)_{5}\right]_{3} \mathrm{~N}$} & & & & & \\
\hline Tri-n-octylamine & {$\left[\mathrm{CH}_{3}\left(\mathrm{CH}_{2}\right)_{7}\right]_{3} \mathrm{~N}$} & & & & & \\
\hline $\begin{array}{l}\text { Tris(hydroxymethyl) Amino } \\
\text { Methane }\end{array}$ & $\left(\mathrm{CH}_{2} \mathrm{OH}\right)_{3} \mathrm{CNH}_{2}$ & & & & & \\
\hline $\begin{array}{l}\text { Trisodium Hydroxyethyl Ethylene- } \\
\text { Diamine Triacetate (HEDTA) }\end{array}$ & $\mathrm{N}_{2} \mathrm{C}_{2} \mathrm{H}_{4}\left(\mathrm{C}_{2} \mathrm{H}_{2} \mathrm{O}_{2} \mathrm{Na}\right)_{3}\left(\mathrm{C}_{2} \mathrm{H}_{4} \mathrm{OH}\right)$ & & & & & \\
\hline
\end{tabular}


Table 1. Summary List of Chemicals Used at Hanford Site Production Plants and Support Operations that Stored Waste in the Single-Shell Tanks (1944-1980). (Sheet 12 of 16)

\begin{tabular}{|c|c|c|c|c|c|c|}
\hline Compcund name & Formala & RCRA & CERCLA & WAC & OSHA & $\begin{array}{l}\text { OES } \\
\text { TLV }\end{array}$ \\
\hline Urea & $\mathrm{CO}\left(\mathrm{NH}_{2}\right)_{2}$ & & & & & \\
\hline Vanadium & $\mathbf{V}$ & & & & & \\
\hline Vanadium Pentoxide & $\mathrm{V}_{2} \mathrm{O}_{5}$ & $\mathrm{X}$ & $\mathrm{X}$ & $\mathrm{X}$ & $\mathbf{X}$ & $\mathrm{X}$ \\
\hline Xylene & $\mathrm{C}_{6} \mathrm{H}_{4}\left(\mathrm{CH}_{3}\right)_{2}$ & $\mathrm{X}$ & $\mathbf{X}$ & $\mathrm{X}$ & $\mathrm{X}$ & $\mathbf{X}$ \\
\hline Yttrium Nitrate & $\mathrm{Y}\left(\mathrm{NO}_{3}\right)_{3}$ & & & & & \\
\hline Zinc Amalgam & $\mathrm{ZnHg}$ & $\mathbf{X}$ & $\mathbf{X}$ & $\mathrm{X}$ & $\mathbf{X}$ & $\mathbf{X}$ \\
\hline Zinc Chloride & $\mathrm{ZnCl}_{2}$ & & $\mathrm{X}$ & & $\mathbf{X}$ & $\mathbf{X}$ \\
\hline Zinc Nitrate & $\mathrm{Zn}\left(\mathrm{NO}_{3}\right)_{2}$ & & $\mathrm{X}$ & & & \\
\hline Zirconium & $\mathbf{Z r}$ & & & & $\mathbf{X}$ & $\mathbf{X}$ \\
\hline Zirconyl Nitrate & $\mathrm{ZrO}\left(\mathrm{NO}_{3}\right)_{2}$ & & & & $\mathrm{X}$ & $\mathrm{X}$ \\
\hline Zirconyl Phosphate & $\mathrm{ZrOPO}_{4}$ & & & & $\mathbf{X}$ & $\mathbf{X}$ \\
\hline
\end{tabular}


Table 1. Summary List of Chemicals Used at Hanford Site Production Plants and Support Operations that Stored Waste in the Single-Shell Tanks (1944-1980). (Sheet 13 of 16)

\begin{tabular}{|c|c|c|c|c|c|c|}
\hline Miscellaneous & Commentsa & RCRA & CERCLA & WAC & OSHA & $\begin{array}{l}\text { OES } \\
\text { TLV }\end{array}$ \\
\hline American Cyanamid S 4058 FLOC & Product Name & & & & & \\
\hline Anti-Foam 60 & Product Name & & & & & \\
\hline Arsenazo III & Contains Arsenic & $\mathbf{X}$ & $\mathbf{X}$ & $\mathbf{X}$ & $\mathbf{X}$ & $\mathbf{X}$ \\
\hline Buffer Solution & Phosphate/Acetate Base & & & & & \\
\hline Clayton Kerful Kleaner & $\mathrm{NaOH}$ & & $\mathbf{X}$ & & $\mathbf{X}$ & $\mathbf{X}$ \\
\hline Diversy Chemical 159 & Product Name & & & & & \\
\hline Dow N-20 FLOC & Product Name & & & & & \\
\hline Dow XD 7889 FLOC & Product Name & & & & & \\
\hline Dow Anti-Foam B & Silicon Emulsion & & & & & \\
\hline Dowex 50 (IX Resin) & Sulfonate Polystyrene DVB & & & & & \\
\hline $\begin{array}{l}\text { Dowex } 21 \mathrm{~K} / \text { Amberlite XE-270 } \\
\text { (IX Resin) }\end{array}$ & $\begin{array}{l}\text { Styrene DVB with Trimethyl } \\
\text { Ammonium }\end{array}$ & & & & & \\
\hline Duolite ARC-359 (IX Resin) & Sulfonated Phenolic & & & & & \\
\hline Hyflo-Super-Cel & Contains Silica & & & & $\mathbf{X}$ & $\mathbf{X}$ \\
\hline Immunol 1468-2 & Product Name & & & & & \\
\hline $\begin{array}{l}\text { Ionac A-580/Permutit SK } \\
\text { (IX Resin) }\end{array}$ & $\begin{array}{l}\text { Polyvinyl Pyridine with Methyl } \\
\text { Groups }\end{array}$ & & & & & \\
\hline Jasco Paint Stripper & Methylene Chloride, Methanol & $\mathrm{X}$ & $\mathrm{X}$ & $\mathbf{X}$ & $\mathbf{X}$ & $\mathbf{X}$ \\
\hline Keolite 25E & Product Name & & & & & \\
\hline Keraff & Product Name & & & & & \\
\hline
\end{tabular}

aRegulation and exposure identifications are based on these substances. 
Table 1. Summary List of Chemicals Used at Hanford Site Production Plants and Support Operations that Stored Waste in the Single-Shell Tanks (1944-1980). (Sheet 14 of 16)

\begin{tabular}{|c|c|c|c|c|c|c|}
\hline Miscellaneous & Commentsa & RCRA & CERCLA & WAC & OSHA & $\begin{array}{l}\text { OES } \\
\text { TLV }\end{array}$ \\
\hline Kleeno Bowl & $\mathrm{HCl}, \mathrm{NH}_{4} \mathrm{Cl}$ & & $\mathbf{X}$ & & $\mathbf{X}$ & $\mathbf{X}$ \\
\hline Magniflox 581-C & Product Name & & & & & \\
\hline Mineral Oil & Light Hydrocarbons & & & & & \\
\hline Mistron & $\mathrm{MgSiO}_{3}$ & & & & $\mathbf{X}$ & $\mathbf{X}$ \\
\hline Oakite Clear Guard & Product Name & & & & & \\
\hline Oakite LSD & $\mathrm{NaOH}$ & & $\mathbf{X}$ & & $\mathbf{X}$ & $\mathbf{X}$ \\
\hline Oakite Rust Stripper & Product Name & & & & & \\
\hline Oakite Swiff & Trichloroethane & $\mathbf{X}$ & $\mathbf{X}$ & $\mathbf{X}$ & $\mathbf{X}$ & $\mathbf{X}$ \\
\hline Orvus $\mathrm{K}$ & Product Name & & & & & \\
\hline Pace-S-Teen & Product Name & & & & & \\
\hline Penvert 192 & Product Name & & & & & \\
\hline Perokleen & Product Name & & & & & \\
\hline Portland Cement & $\begin{array}{l}\mathrm{CaSi}(\mathrm{OH})_{2}, \mathrm{CaO} \cdot \mathrm{SiO}_{2}, \\
\mathrm{CaO} \cdot \mathrm{Al}_{2} \mathrm{O}_{3} \cdot \mathrm{Fe}_{2} \mathrm{O}_{3}\end{array}$ & & & & $\mathbf{X}$ & $\mathbf{X}$ \\
\hline Resin IRN-150 & Styrene Divinyl Benzene & & $\because$ & & & \\
\hline Saf-tee Solvent F.O. 128 & Product Name & & & & & \\
\hline Sani-Flush & $\mathrm{NH}_{4} \mathrm{Cl}$, Diethylthiourea & & $\mathbf{X}$ & & $\mathbf{X}$ & $\mathbf{X}$ \\
\hline Shell Spray Base & Kerosene & & & & & \\
\hline Shell E-2342 & Naphthalene and Paraffins & $\mathbf{X}$ & $\mathbf{X}$ & $\mathbf{X}$ & $\mathbf{X}$ & $\mathbf{X}$ \\
\hline Soltrol-170 & Paraffins & & & & & \\
\hline
\end{tabular}

a Regulation and exposure identifications are based on these substances. 
Table 1. Summary List of Chemicals Used at Hanford Site Production Plants and Support Operations that Stored Waste in the Single-Shell Tanks (1944-1980). (Sheet 15 of 16)

\begin{tabular}{|c|c|c|c|c|c|c|}
\hline Miscellaneous & Commentsa & RCRA & CERCLA & WAC & OSHA & $\begin{array}{l}\text { OES } \\
\text { TLV }\end{array}$ \\
\hline Spartan DC 13 & No Hazardous Compounds & & & & & \\
\hline Spic-n-Span (Detergent) & $\mathrm{Na}_{3} \mathrm{PO}_{4}, \mathrm{Na}_{2} \mathrm{SO}_{4}, \mathrm{Na}$ Silicates & & $\mathbf{X}$ & & & \\
\hline Tide (Detergent) & $\mathrm{Na}_{3} \mathrm{PO}_{4}, \mathrm{Na}_{2} \mathrm{CO}_{3}, \mathrm{Na}$ Silicates & & $\mathbf{X}$ & & & \\
\hline Turco (Fabrifilm) & $\begin{array}{l}\text { Toluene, Butanol, Isopropanol, } \\
\text { Acetone }\end{array}$ & $\mathbf{X}$ & $\mathbf{X}$ & $\mathbf{X}$ & $\mathbf{X}$ & $\mathbf{X}$ \\
\hline Turco EPO Strip & Product Name & & & & & \\
\hline Turco EPO Strip NP & Product Name & & & & & \\
\hline Turco 2822 & $\begin{array}{l}\text { Methylene Chloride, } \\
\text { Acetic Acid }\end{array}$ & $\mathrm{X}$ & $\mathrm{X}$ & $\mathbf{X}$ & $\mathbf{X}$ & $\mathbf{X}$ \\
\hline Turco 2844 & Product Name & & & & & \\
\hline Turco 4306 B,C,\&D & $\mathrm{NaHSO}_{4}, \mathrm{NH}_{2} \mathrm{SO}_{3} \mathrm{H}$ & & & & & \\
\hline Turco 4358-4A & Product Name & & & & & \\
\hline Turco $4501 \mathrm{~A}$ & $\mathrm{KOH}$, Diamine Compounds & & $\mathbf{X}$ & & $\mathbf{X}$ & $\mathbf{X}$ \\
\hline Turco $4502 \mathrm{D}$ & $\mathrm{KOH}, \mathrm{K}_{2} \mathrm{CrO}_{4}, \mathrm{KMnO}_{4}$ & $\mathbf{X}$ & $\mathbf{X}$ & $\mathbf{X}$ & $\mathbf{X}$ & $\mathbf{X}$ \\
\hline Turco 4512 A & $\mathrm{H}_{3} \mathrm{PO}_{4}$ & & $\mathbf{X}$ & & $\mathbf{X}$ & $\mathbf{X}$ \\
\hline Turco 4518 & $\begin{array}{l}\mathrm{HO}_{2} \mathrm{CCO}_{2} \mathrm{H} \cdot 2 \mathrm{H}_{2} \mathrm{O}, \text { Sodium } \\
\text { Dodecyl Bezene Sulfonate }\end{array}$ & & $\mathbf{X}$ & & $\mathbf{X}$ & $\mathbf{X}$ \\
\hline Turco 4521 & $\begin{array}{l}\left(\mathrm{NH}_{4}\right)_{2} \mathrm{C}_{2} \mathrm{O}_{4}, \mathrm{HO}_{2} \mathrm{CCO}_{2} \mathrm{H} \cdot 2 \mathrm{H}_{2} \mathrm{O}, \\
\text { Sodium Dodecyl Benene } \\
\text { Sulfonate }\end{array}$ & & $\mathbf{X}$ & & $\mathbf{X}$ & $\mathbf{X}$ \\
\hline Turco 4605-8 & Product Name & & & & & \\
\hline
\end{tabular}

aRegulation and exposure identifications are based on these substances. 
Table 1. Summary List of Chemicals Used at Hanford Site Production Plants and Support Operations that Stored Waste in the Single-Shell Tanks (1944-1980). (Sheet 16 of 16)

\begin{tabular}{|c|c|c|c|c|c|c|}
\hline Miscellaneous & Commentsa & RCRA & CERCLA & WAC & OSHA & $\begin{array}{l}\text { OES } \\
\text { TLV }\end{array}$ \\
\hline Turco 4669 & Product Name & & & & & \\
\hline Turco 4715 & Product Name & & & & & \\
\hline Turco 4738 (Thin! & Product Name & & & & & \\
\hline Turco T-5561 & $\begin{array}{l}\text { 2-Butoxyethanol, } \\
\text { Dioctylphthalate, Morpholine, } \\
\text { Mineral Oil }\end{array}$ & $\mathbf{X}$ & $\mathbf{X}$ & $\mathbf{X}$ & $\mathbf{X}$ & $\mathbf{X}$ \\
\hline Turco T-5589 & Isopropanol, $\mathrm{NH}_{4} \mathrm{OH}$ & & $\mathbf{X}$ & & $\mathbf{X}$ & $\mathbf{X}$ \\
\hline Turco Alkaline (Fust Remover) & $\mathrm{NaOH}$, Kerosene & & $\mathbf{X}$ & & $\mathbf{X}$ & $\mathbf{X}$ \\
\hline Turco Deseal Zit 2 & $\begin{array}{l}\text { Methylene Chloride, Acetic } \\
\text { Acid }\end{array}$ & $\mathbf{X}$ & $\mathbf{X}$ & $\mathbf{X}$ & $\mathbf{X}$ & $\mathrm{X}$ \\
\hline Turco Plaudit & No Hazardous Compounds & & & & & \\
\hline West Lode Degreaser & $\begin{array}{l}\text { Contains Aromatic } \\
\text { Hydrocarbons }\end{array}$ & $\mathbf{X}$ & $\mathbf{X}$ & $\mathbf{X}$ & $\mathbf{X}$ & $\mathbf{X}$ \\
\hline Wyandotte Kelvar & Product Name & & & & & \\
\hline Wyandotte MF & Product Name & & & & & \\
\hline Wyandotte P1075 & Product Name & & & & & \\
\hline W'yandotte 1112 & Product Name & & & & & \\
\hline Zeolite AW-500 (IX Resin) & $\mathrm{Al}_{2}\left(\mathrm{SiO}_{5}\right)_{2}(\mathrm{OH})_{2}$ & & & & & \\
\hline Zeolon 900 (IX Resin) & $\mathrm{Al}_{2}\left(\mathrm{SiO}_{5}\right)_{2}(\mathrm{OH})_{2}$ & & & & & \\
\hline
\end{tabular}

aRegulation and exposure identifications are based on these substances. 
The following tables represent the list of chemicals used by each facility that stored waste in the SSTs.

Table 2. N Reactor Decontamination (100 Area) (1972-1976).

\begin{tabular}{|l|l|l|}
\hline \multicolumn{1}{|c|}{ Compound name } & \multicolumn{1}{c|}{ Formula } & \multicolumn{1}{c|}{ Comments } \\
\hline Ammonia Bisulfate & $\mathrm{NH}_{4} \mathrm{HSO}_{4} \cdot \mathrm{H}_{2} \mathrm{O}$ & \\
\hline Citric Acid & $\mathrm{C}_{3} \mathrm{H}_{4} \mathrm{OH}(\mathrm{COOH})_{3}$ & \\
\hline Hydrazine & $\mathrm{H}_{2} \mathrm{NNH}_{2} \cdot \mathrm{H}_{2} \mathrm{O}$ & \\
\hline Morpholine & $\mathrm{C}_{4} \mathrm{H}_{9} \mathrm{NO}$ & Water treatment \\
\hline Oxalic Acid & $\mathrm{HO}_{2} \mathrm{CCO}_{2} \mathrm{H} \cdot 2 \mathrm{H}_{2} \mathrm{O}$ & \\
\hline Potassium Permanganate & $\mathrm{KMnO}_{4}$ & \\
\hline Sodium Hydroxide & $\mathrm{NaOH}$ & \\
\hline Sodium Nitrite & $\mathrm{NaNO}_{2}$ & \\
\hline Turco 4512 A & $\mathrm{H}_{3} \mathrm{PO}_{4}$ & Product name \\
\hline
\end{tabular}


Table 3. PUREX Plant (A Plant): Fuel Reprocessing (200 East Area) (1955-1972). (Sheet 1 of 2)

\begin{tabular}{|c|c|c|}
\hline Compound name & Formula & Comments \\
\hline Aluminum & $\mathrm{Al}$ & Fuel element constituent \\
\hline $\begin{array}{l}\text { Aluminum Nitrate } \\
\text { Nonahydrate }\end{array}$ & $\mathrm{Al}\left(\mathrm{NO}_{3}\right)_{3} \cdot 9 \mathrm{H}_{2} \mathrm{O}$ & \\
\hline $\begin{array}{l}\text { Aluminum Nitrate (Mono } \\
\text { Basic) }\end{array}$ & $\mathrm{Al}(\mathrm{OH})\left(\mathrm{NO}_{3}\right)_{2}$ & \\
\hline Ammonium Fluoride & $\mathrm{NH}_{4} \mathrm{~F}$ & \\
\hline Ammonium Nitrate & $\mathrm{NH}_{4} \mathrm{NO}_{3}$ & \\
\hline Beryllium & $\mathrm{Be}$ & Fuel element constituent \\
\hline Cadmium Nitrate & $\mathrm{Cd}\left(\mathrm{NO}_{3}\right)_{2}$ & \\
\hline Dibutyl Butyl Phosphonate & $\begin{array}{l}\mathrm{CH}_{3}\left(\mathrm{CH}_{2}\right)_{3} \mathrm{PO}_{3} \\
{\left[\mathrm{CH}_{3}\left(\mathrm{CH}_{2}\right)_{3}\right]_{2}}\end{array}$ & \\
\hline DOW Anti-Foam B & Silicon Emulsion & \\
\hline $\begin{array}{l}\text { Dowex } 21 \text { K/Amberlite XE- } \\
270 \text { (IX Resin) }\end{array}$ & $\begin{array}{l}\text { Polystyrene DVB } \\
\text { with Trimethyl } \\
\text { Ammonia }\end{array}$ & Product name \\
\hline Ferric Nitrate & $\mathrm{Fe}\left(\mathrm{NO}_{3}\right)_{3} \cdot 6 \mathrm{H}_{2} \mathrm{O}$ & \\
\hline Ferrous Sulfamate & $\mathrm{Fe}\left(\mathrm{SO}_{3} \mathrm{NH}_{2}\right)_{2}$ & \\
\hline Formaldehyde (Solution) & $\mathrm{CH}_{2} \mathrm{O}$ & \\
\hline Hydrazine & $\mathrm{H}_{2} \mathrm{NNH}_{2} \cdot \mathrm{H}_{2} \mathrm{O}$ & \\
\hline Hydroxylamine Nitrate & $\mathrm{NH}_{2} \mathrm{OH} \cdot \mathrm{HNO}_{3}$ & \\
\hline $\begin{array}{l}\text { Ionac A-580/Permutit SK } \\
\text { (IX Resin) }\end{array}$ & $\begin{array}{l}\text { Polyvinyl Puridine } \\
\text { with Methyl Groups }\end{array}$ & Product name \\
\hline Iron & $\overline{\mathrm{Fe}}$ & Fuel element constituent \\
\hline Mercuiric Nitrate & $\mathrm{Hg}\left(\mathrm{NO}_{3}\right)_{2} \cdot \mathrm{H}_{2} \mathrm{O}$ & \\
\hline Nitric Acid & $\mathrm{HNO}_{3}$ & \\
\hline $\begin{array}{l}\text { Normal Paraffin } \\
\text { Hydrocarbon }\end{array}$ & $\mathrm{C}_{10} \mathrm{H}_{22}$ to $\mathrm{C}_{14} \mathrm{H}_{30}$ & \\
\hline Oxalic Acid & $\mathrm{HO}_{2} \mathrm{CCO}_{2} \mathrm{H} \cdot 2 \mathrm{H}_{2} \mathrm{O}$ & \\
\hline Phosphoric Acid & $\mathrm{H}_{3} \mathrm{PO}_{4}$ & \\
\hline Potassium Fluoride & $\mathrm{KF}$ & \\
\hline Potassium Hydroxide & $\mathrm{KOH}$ & \\
\hline Potassium Permanganate & $\mathrm{KMnO}_{4}$ & \\
\hline Shell E-2342 & $\begin{array}{l}\text { Naphthalene and } \\
\text { Paraffins }\end{array}$ & Product name \\
\hline
\end{tabular}


Table 3. PUREX Plant (A Plant): Fuel Reprocessing (200 East Area) (1955-1972). (Sheet 2 of 2)

\begin{tabular}{|l|l|l|}
\hline \multicolumn{1}{|c|}{ Compound name } & \multicolumn{1}{c|}{ Formula } & \multicolumn{1}{c|}{ Comments } \\
\hline Silicon & $\mathrm{Si}$ & Fuel element constituent \\
\hline Silver Nitrate & $\mathrm{AgNO}_{3}$ & \\
\hline Sodium Carbonate & $\mathrm{Na}_{2} \mathrm{CO}_{3}$ & \\
\hline Sodium Fluoride & $\mathrm{NaF}$ & \\
\hline Sodium Hydroxide & $\mathrm{NaOH}$ & \\
\hline Sodium Nitrate & $\mathrm{NaNO}_{3}$ & \\
\hline Sodium Nitrite & $\mathrm{NaNO}_{2}$ & \\
\hline Sodium Thiosulfate & $\mathrm{Na}_{2} \mathrm{~S}_{2} \mathrm{O}_{3}$ & \\
\hline Soltrol-170 & $\mathrm{C}_{10} \mathrm{H}_{22}$ to $_{16} \mathrm{H}_{34}$ & Product name \\
\hline Sugar & $\mathrm{C}_{12} \mathrm{H}_{22} \mathrm{O}_{11}$ & \\
\hline Sulfamic Acid & $\mathrm{NH}_{2} \mathrm{SO}_{3} \mathrm{H}$ & \\
\hline Sulfuric Acid & $\mathrm{H}_{2} \mathrm{SO}_{4}$ & \\
\hline Tartaric Acid & $\mathrm{C}_{2} \mathrm{H}_{2}\left(\mathrm{OHCOOH}_{2}\right.$ & \\
\hline Tributyl Phosphate & $\left(\mathrm{C}_{4} \mathrm{H}_{9}\right)_{3} \mathrm{PO}_{4}$ & \\
\hline Trichloroethane & $\mathrm{CH}_{3} \mathrm{CHCl}_{3}$ & Equipment degreasing \\
\hline Tri-n-dodecylamine & {$\left[\mathrm{CH}_{3}\left(\mathrm{CH}_{2}\right)_{11}\right]_{3} \mathrm{~N}$} & \\
\hline Zirconium & $\mathrm{Zr}$ & Fuel element constituent \\
\hline
\end{tabular}


WHC-EP-0172 Revision I

Table 4. PUREX Plant (A Plant): Analytical Laboratory (200 East Area) (1955-1972).

\begin{tabular}{|c|c|c|}
\hline Compound name & Formula & Comments \\
\hline Acetone & $\mathrm{CH}_{3} \mathrm{C}_{2} \mathrm{OH}_{3}$ & . \\
\hline Carbon Tetrachloride & $\mathrm{CCl}_{4}$ & \\
\hline Ceric Sulfate & $\mathrm{Ce}\left(\mathrm{SO}_{4}\right)_{2}$ & \\
\hline Ceric Fluoride & $\mathrm{CeF}_{4}$ & \\
\hline Ferrous Sulfate & $\mathrm{FeSO}_{4}$ & \\
\hline Hydrobromic Acid & $\mathrm{HBr}$ & \\
\hline Hydrochloric Acid & $\mathrm{HCl}$ & \\
\hline Hydrogen Peroxide & $\mathrm{H}_{2} \mathrm{O}_{2}$ & \\
\hline $\begin{array}{l}\text { Hydroxylamine } \\
\text { Hydrochloride }\end{array}$ & $\mathrm{NH}_{2} \mathrm{OH} \cdot \mathrm{HCl}$ & 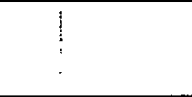 \\
\hline Isopropyl Alcohol & $\mathrm{C}_{3} \mathrm{H}_{7} \mathrm{OH}$ & \\
\hline Lanthanum Fluoride & $\mathrm{LaF}_{3}$ & \\
\hline Lanthanum Hydroxide & $\mathrm{La}(\mathrm{OH})_{3}$ & \\
\hline Lanthanum Nitrate & $\mathrm{La}\left(\mathrm{NO}_{3}\right)_{3}$ & \\
\hline Magnesium & $\mathrm{Mg}$ & \\
\hline Nitric Acid & $\mathrm{HNO}_{3}$ & \\
\hline Periodic Acid & $\mathrm{HIO}_{4}$ & \\
\hline Phosphorous Pentoxide & $\mathrm{P}_{2} \mathrm{O}_{5}$ & \\
\hline Potassium Oxalate & $\mathrm{K}_{2} \mathrm{C}_{2} \mathrm{O}_{4}$ & \\
\hline Potassium Permanganate & $\mathrm{KMnO}_{4}$ & \\
\hline Silver Nitrate & $\mathrm{AgNO}_{3}$ & \\
\hline Sodium Bisulfate & $\mathrm{NaHSO}_{4} \cdot \mathrm{H}_{2} \mathrm{O}$ & \\
\hline Sodium Bromate & $\mathrm{NaBrO}_{3}$ & \\
\hline Sodium Carbonate & $\mathrm{Na}_{2} \mathrm{CO}_{3}$ & \\
\hline Sodium Fluoride & $\mathrm{NaF}$ & \\
\hline Sodium Hydroxide & $\mathrm{NaOH}$ & \\
\hline Sodium Nitrate & $\mathrm{NaNO}_{3}$ & \\
\hline Sulfuric Acid & $\mathrm{H}_{2} \mathrm{SO}_{4}$ & \\
\hline Thenoyltrifluoroacetone & $\begin{array}{l}(\mathrm{CH})_{3} \mathrm{SCOCH}_{2} \\
\mathrm{COCF}_{3} \\
\end{array}$ & \\
\hline Zirconyl Phosphate & $\mathrm{ZrOPO}_{4}$ & \\
\hline
\end{tabular}


WHC-EP-0172 Revision 1

Table 5. PUREX Plant (A Plant): Fission Product Recovery (200 East Area) (1960-1966).

\begin{tabular}{|l|l|l|}
\hline \multicolumn{1}{|c|}{ Compound name } & \multicolumn{1}{c|}{ Formula } & Comments \\
\hline Acetic Acid & $\mathrm{CH}_{3} \mathrm{CO}_{2} \mathrm{H}$ & \\
\hline Ferric Nitrate & $\mathrm{Fe}\left(\mathrm{NO}_{3}\right)_{3} \cdot 6 \mathrm{H}_{2} \mathrm{O}$ & \\
\hline Hydroxyacetic Acid & $\mathrm{CH}_{3} \mathrm{OCOOH}$ & \\
\hline Lead Nitrate & $\mathrm{Pb}\left(\mathrm{NO}_{3}\right)_{2}$ & \\
\hline Nickel Nitrate & $\left.\mathrm{Ni}_{(\mathrm{NO}}\right)_{2}$ & \\
\hline Nitric Acid & $\mathrm{HNO}_{3}$ & \\
\hline Oxalic Acid & $\mathrm{HO}_{2} \mathrm{CCO}_{2} \mathrm{H} \cdot 2 \mathrm{H}_{2} \mathrm{O}$ & \\
\hline Silver Nitrate & $\mathrm{AgNO}_{3}$ & \\
\hline Sodium Carbonate & $\mathrm{Na}_{2} \mathrm{CO}_{3}$ & \\
\hline Sodium Ferrocyanide & $\mathrm{Na}_{4} \mathrm{Fe}_{(\mathrm{CN})_{6}}$ & \\
\hline Sodium Hydroxide & $\mathrm{NaOH}$ & \\
\hline Sodium Nitrate & $\mathrm{NaNO}_{3}$ & \\
\hline Sodium Sulfate & $\mathrm{Na}_{2} \mathrm{SO}_{4}$ & \\
\hline Tartaric Acid & $\mathrm{C}_{2} \mathrm{H}_{2}(\mathrm{OHCOOH})_{2}$ & \\
\hline
\end{tabular}


WHC-EP-0172 Revision 1

Table 6. B Plant: Fuel Reprocessing (200 East Area) (1945-1952).

\begin{tabular}{|c|c|c|}
\hline Compound name & Formula & Comments \\
\hline Aluminum & $\mathrm{Al}$ & Fuel element constituent \\
\hline Ammonium Nitrate & $\mathrm{NH}_{4} \mathrm{NO}_{3}$ & \\
\hline Ammonium Silicofluoride & $\left(\mathrm{NH}_{4}\right)_{2} \mathrm{SiF}_{6}$ & \\
\hline Ammonium Sulfate & $\left(\mathrm{NH}_{4}\right)_{2} \mathrm{SO}_{4}$ & \\
\hline Ammonium Sulfite & $\left(\mathrm{NH}_{4}\right)_{2} \mathrm{SO}_{3}$ & \\
\hline Ceric Nitrate & $\mathrm{Ce}\left(\mathrm{NO}_{3}\right)_{4}$ & \\
\hline Ferrous Sulfate & $\mathrm{FeSO}_{4}$ & \\
\hline Hydrogen Fluoride & $\mathrm{HF}$ & \\
\hline Hydrogen Peroxide & $\mathrm{H}_{2} \mathrm{O}_{2}$ & \\
\hline Hyflo-Super-Cel & Contains Silica & Product name \\
\hline Lanthanum Nitrate & $\mathrm{La}\left(\mathrm{NO}_{3}\right)_{3}$ & \\
\hline Nitric Acid & $\mathrm{HNO}_{3}$ & \\
\hline Oxalic Acid & $\mathrm{HO}_{2} \mathrm{CCO}_{2} \mathrm{H} \cdot 2 \mathrm{H}_{2} \mathrm{O}$ & \\
\hline Phosphoric Acid & $\mathrm{H}_{3} \mathrm{PO}_{4}$ & \\
\hline Potassium Hydroxide & KOH & \\
\hline Potassium Permanganate & $\mathrm{KMnO}_{4}$ & \\
\hline Silicon & Si & Fuel element constituent \\
\hline Sodium Bismuthate & $\mathrm{NaBiO}_{3}$ & \\
\hline Sodium Carbonate & $\mathrm{Na}_{2} \mathrm{CO}_{3}$ & \\
\hline Sodium Dichromate & $\mathrm{Na}_{2} \mathrm{Cr}_{2} \mathrm{O}_{7} \cdot 2 \mathrm{H}_{2} \mathrm{O}$ & \\
\hline Sodium Hydroxide & $\mathrm{NaOH}$ & \\
\hline Sodium Nitrate & $\mathrm{NaNO}_{3}$ & \\
\hline Sodium Nitrite & $\mathrm{NaNO}_{2}$ & \\
\hline Sulfuric Acid & $\mathrm{H}_{2} \mathrm{SO}_{4}$ & \\
\hline Trichloroethane & $\mathrm{CH}_{3} \mathrm{CHCl}_{3}$ & Equipment degreasing \\
\hline Zirconyl Nitrate & $\mathrm{ZrO}\left(\mathrm{NO}_{3}\right)_{2}$ & \\
\hline
\end{tabular}


WHC-EP-0172 Revision 1

Table 7. B Plant: Strontium Recovery and Waste Fractionization (200 East Area) (1965-1976). (Sheet 1 of 2)

\begin{tabular}{|c|c|c|}
\hline Compound name & Formula & Comments \\
\hline $\begin{array}{l}\text { Aluminum Nitrate } \\
\text { Nonahydrate }\end{array}$ & $\mathrm{AL}\left(\mathrm{NO}_{3}\right)_{3} \cdot 9 \mathrm{H}_{2} \mathrm{O}$ & \\
\hline Ammonia (Anhydrous) & $\mathrm{NH}_{3}$ & \\
\hline Calcium Carbonate & $\mathrm{CaCO}_{3}$ & \\
\hline Carbon Dioxide & $\mathrm{CO}_{2}$ & \\
\hline Chromium Nitrate & $\mathrm{Cr}\left(\mathrm{NO}_{3}\right)_{3}$ & \\
\hline Citric Acid & $\mathrm{C}_{3} \mathrm{H}_{4} \mathrm{OH}(\mathrm{COOH})_{3}$ & \\
\hline $\begin{array}{l}\text { Di2-Ethyl Hexyl Phosphoric } \\
\text { Acid }\end{array}$ & $\mathrm{C}_{16} \mathrm{H}_{34} \mathrm{O}_{2} \mathrm{POOH}$ & . \\
\hline Duolite ARC-359 (IX Resin) & Sulfonated Phenolic & Product name \\
\hline Ferric Nitrate & $\mathrm{Fe}\left(\mathrm{NO}_{3}\right)_{3} \cdot 6 \mathrm{H}_{2} \mathrm{O}$ & \\
\hline Hydrogen Peroxide & $\mathrm{H}_{2} \mathrm{O}_{2}$ & \\
\hline Hydroxyacetic Acid & $\mathrm{CH}_{3} \mathrm{OCOOH}$ & \\
\hline $\begin{array}{l}\text { Lanthanum-Neodynium } \\
\text { Nitrate }\end{array}$ & $\mathrm{La}\left(\mathrm{NO}_{3}\right)_{3}-\mathrm{Nd}\left(\mathrm{NO}_{3}\right)_{2}$ & \\
\hline Lead Nitrate & $\mathrm{Pb}\left(\mathrm{NO}_{3}\right)_{2}$ & \\
\hline Magnesium Nitrate & $\mathrm{Mg}\left(\mathrm{NO}_{3}\right)_{2}$ & \\
\hline Magnesium Carbonate & $\mathrm{MgCO}_{3}$ & \\
\hline Nickel Nitrate & $\mathrm{Ni}\left(\mathrm{NO}_{3}\right)_{2} \cdot 6 \mathrm{H}_{2} \mathrm{O}$ & \\
\hline Nitric Acid & $\mathrm{HNO}_{3}$ & \\
\hline $\begin{array}{l}\text { Normal Paraffin } \\
\text { Hydrocarbon }\end{array}$ & $\mathrm{C}_{10} \mathrm{H}_{22}$ to $\mathrm{C}_{14} \mathrm{H}_{30}$ & \\
\hline Oxalic Acid & $\mathrm{HO}_{2} \mathrm{CCO}_{2} \mathrm{H} \cdot 2 \mathrm{H}_{2} \mathrm{O}$ & \\
\hline Phosphotungstic Acid & $\begin{array}{l}\mathrm{H}_{3}\left[\mathrm{P}\left(\mathrm{W}_{3} \mathrm{O}_{10}\right)_{4}\right] \\
14 \mathrm{H}_{2} \mathrm{O}\end{array}$ & \\
\hline Potassium Permanganate & $\mathrm{KMnO}_{4}$ & \\
\hline Silver Nitrate & $\mathrm{Ag}\left(\mathrm{NO}_{3}\right)_{2}$ & \\
\hline Sodium Acetate & $\mathrm{NaC}_{2} \mathrm{H}_{3} \mathrm{O}_{2} \cdot 3 \mathrm{H}_{2} \mathrm{O}$ & \\
\hline Sodium Bisulfate & $\mathrm{NaHSO}_{4}$ & \\
\hline Sodium Carbonate & $\mathrm{Na}_{2} \mathrm{CO}_{3}$ & \\
\hline Sodium Gluconate & $\begin{array}{l}(\mathrm{CHOH})_{4} \mathrm{CO}_{2} \mathrm{HCH}_{2} \\
\mathrm{ONa}\end{array}$ & \\
\hline Sodium Hydroxide & $\mathrm{NaOH}$ & \\
\hline
\end{tabular}


WHC-EP-0172 Revision 1

Table 7. B Plant: Strontium Recovery and Waste Fractionization (200 East Area) (1965-1976). (Sheet 2 of 2)

\begin{tabular}{|l|l|l|}
\hline \multicolumn{1}{|c|}{ Compound name } & \multicolumn{1}{c|}{ Formula } & \multicolumn{1}{c|}{ Comments } \\
\hline Sodium Nitrate & $\mathrm{NaNO}_{3}$ & \\
\hline Sodium Nitrite & $\mathrm{NaNO}_{2}$ & \\
\hline Sodium Persulfate & $\mathrm{Na}_{2} \mathrm{~S}_{2} \mathrm{O}_{8}$ & \\
\hline Sodium Phosphate & $\mathrm{Na}_{3} \mathrm{PO}_{4}$ & \\
\hline Sodium Sulfate & $\mathrm{Na}_{2} \mathrm{SO}_{4}$ & \\
\hline Sugar & $\mathrm{C}_{12} \mathrm{H}_{22} \mathrm{O}_{11}$ & \\
\hline Tartaric Acid & $\mathrm{C}_{2} \mathrm{H}_{2}\left(\mathrm{OHCOOH}_{2}\right.$ & \\
\hline $\begin{array}{l}\text { Tetrasodium Ethylene } \\
\text { Diamine-Tetra Acetate } \\
\text { (EDTA) }\end{array}$ & $\mathrm{N}_{2} \mathrm{C}_{2} \mathrm{H}_{4}\left(\mathrm{C}_{2} \mathrm{H}_{2} \mathrm{O}_{2} \mathrm{Na}\right)_{4}$ & \\
\hline Tributyl Phosphate & & \\
\hline Trichloroethane & $\left(\mathrm{C}_{4} \mathrm{H}_{9}\right)_{3} \mathrm{PO}{ }_{4}$ & \\
\hline $\begin{array}{l}\text { Trisodium Hydroxyethyl } \\
\text { Ethylene-Diamine } \\
\text { Triacetate (HEDTA) }\end{array}$ & $\mathrm{CH}_{3} \mathrm{CHCl}_{3}$ & Equipment degreasing \\
\hline Zeolite AW-500 (IX Resin) & $\mathrm{N}_{2} \mathrm{C}_{2} \mathrm{H}_{4}\left(\mathrm{C}_{2} \mathrm{H}_{2} \mathrm{O}_{2} \mathrm{Na}\right)_{3}$ & \\
$\left(\mathrm{C}_{2} \mathrm{H}_{4} \mathrm{OH}\right)_{2}$ & $\mathrm{Al}_{2}\left(\mathrm{SiO}_{5}\right)_{2}(\mathrm{OH})_{2}$ & Product name \\
\hline
\end{tabular}


WHC-EP-0172 Revision 1

Table 8. B Plant: Waste Encapsulation (200 East Area) (1974-1976).

\begin{tabular}{|l|l|l|}
\hline \multicolumn{1}{|c|}{ Compound name } & \multicolumn{1}{c|}{ Formula } & Comments \\
\hline Acetone & $\mathrm{CH}_{3} \mathrm{C}_{2} \mathrm{OH}_{3}$ & \\
\hline Boric Acid & $\mathrm{H}_{3} \mathrm{BO}_{3}$ & \\
\hline Calcium Chloride & $\mathrm{CaCl}_{2}$ & \\
\hline Cesium Chloride & $\mathrm{CsCl}$ & \\
\hline Hydrochloric Acid & $\mathrm{HCl}$ & \\
\hline Hydrogen Peroxide & $\mathrm{H}_{2} \mathrm{O}_{2}$ & \\
\hline Nitric Acid & $\mathrm{HNO}_{3}$ & \\
\hline Oxalic Acid & $\mathrm{HO}_{2} \mathrm{CCO}_{2} \mathrm{H} \cdot 2 \mathrm{H}_{2} \mathrm{O}$ & \\
\hline Phosphoric Acid & $\mathrm{H}_{3} \mathrm{PO}_{4}$ & \\
\hline Potassium Permanganate & $\mathrm{KMnO}_{4}$ & \\
\hline Sodium Fluoride & $\mathrm{NaF}$ & \\
\hline Sodium Hydroxide & $\mathrm{NaOH}$ & \\
\hline Sodium Nitrate & $\mathrm{NaNO}_{3}$ & \\
\hline Sodium Phosphate & $\mathrm{Na}_{3} \mathrm{PO}_{4}$ & \\
\hline Strontium Fluoride & $\mathrm{SrF}_{2}$ & \\
\hline Trichloroethane & $\mathrm{CH}_{3} \mathrm{CCl}_{3}$ & \\
\hline
\end{tabular}


WHC-EP-0172 Revision 1

Table 9. Semiworks Pilot Plant (200 East Area) (1955-1967). (Sheet 1 of 2)

\begin{tabular}{|c|c|c|}
\hline Compound name & Formula & Comments \\
\hline Acetic Acid & $\mathrm{CH}_{3} \mathrm{CO}_{2} \mathrm{H}$ & \\
\hline $\begin{array}{l}\text { Aluminum Nitrate } \\
\text { Nonahydrate }\end{array}$ & $\mathrm{Al}\left(\mathrm{NO}_{3}\right) 3 \cdot 9 \mathrm{H}_{2} \mathrm{O}$ & \\
\hline Calcium Nitrate & $\mathrm{Ca}\left(\mathrm{NO}_{3}\right)_{2}$ & \\
\hline Chromium Nitrate & $\mathrm{Cr}\left(\mathrm{NO}_{3}\right)_{3}$ & \\
\hline Citric Acid & $\left.\mathrm{C}_{3} \mathrm{H}_{4} \mathrm{OH}(\mathrm{COOH})\right)_{2}$ & \\
\hline $\begin{array}{l}\text { Di2-Ethyl Hexyl Phosphoric } \\
\text { Acid }\end{array}$ & $\mathrm{C}_{16} \mathrm{H}_{34} \mathrm{O}_{2} \mathrm{POOH}$ & \\
\hline Ferric Nitrate & $\mathrm{Fe}\left(\mathrm{NO}_{3}\right)_{3} \cdot 6 \mathrm{H}_{2} \mathrm{O}$ & \\
\hline Ferric Sulfate & $\mathrm{Fe}_{2}\left(\mathrm{SO}_{4}\right)_{3}$ & \\
\hline Ferrous Sulfamate & $\mathrm{Fe}\left(\mathrm{SO}_{3} \mathrm{NH}_{2}\right)_{2}$ & \\
\hline Glycolic Acid & $\mathrm{HOCH}_{2} \mathrm{CO}_{2} \mathrm{H}$ & \\
\hline Hydrazine & $\mathrm{H}_{2} \mathrm{NNH}_{2} \cdot \mathrm{H}_{2} \mathrm{O}$ & \\
\hline Hydrogen Peroxide & $\mathrm{H}_{2} \mathrm{O}_{2}$ & \\
\hline Hydroxyacetic Acid & $\mathrm{CH}_{3} \mathrm{OCOOH}$ & \\
\hline Lead Nitrate & $\mathrm{Pb}\left(\mathrm{NO}_{3}\right)_{2}$ & \\
\hline Nickel Nitrate & $\mathrm{Ni}\left(\mathrm{NO}_{3}\right)_{2} \cdot 6 \mathrm{H}_{2} \mathrm{O}$ & \\
\hline Nitric Acid & $\mathrm{HNO}_{3}$ & \\
\hline Nitrilotriacetic Acid (NTA) & $\mathrm{N}\left(\mathrm{CH}_{2} \mathrm{COOH}\right)_{3}$ & \\
\hline $\begin{array}{l}\text { Normal Paraffin } \\
\text { Hydrocarbon }\end{array}$ & $\mathrm{C}_{10} \mathrm{H}_{22}$ to $\mathrm{C}_{14} \mathrm{H}_{30}$ & \\
\hline Oxalic Acid & $\mathrm{HO}_{2} \mathrm{CCO}_{2} \mathrm{H} \cdot 2 \mathrm{H}_{2} \mathrm{O}$ & \\
\hline $\begin{array}{l}\text { Pentasodium Diethylene } \\
\text { Triamine Penta Acetate } \\
\text { (DTPA) }\end{array}$ & $\mathrm{N}_{3}\left(\mathrm{CH}_{2}\right)_{4}(\mathrm{COONa})_{5}$ & \\
\hline Potassium Bicarbonate & $\mathrm{KHCO}_{3}$ & \\
\hline Potassium Permanganate & $\mathrm{KMnO}_{4}$ & \\
\hline Potassium Persulfate & $\mathrm{K}_{2} \mathrm{~S}_{2} \mathrm{O}_{8}$ & \\
\hline Shell Spray Base & $\mathrm{C}_{10} \mathrm{H}_{22}$ to $\mathrm{C}_{16} \mathrm{H}_{34}$ & Product name \\
\hline Shell E-2342 & $\begin{array}{l}\text { Naphthalene and } \\
\text { Paraffins }\end{array}$ & Product name \\
\hline Silver Nitrate & $\mathrm{AgNO}_{3}$ & \\
\hline
\end{tabular}


Table 9. Semiworks Pilot Plant (200 East Area) (1955-1967). (Sheet 2 of 2)

\begin{tabular}{|l|l|l|}
\hline \multicolumn{1}{|c|}{ Compound name } & \multicolumn{1}{|c|}{ Formula } & \multicolumn{1}{c|}{ Comments } \\
\hline Sodium Acetate & $\mathrm{NaC}_{2} \mathrm{H}_{3} \mathrm{O}_{2} \cdot 3 \mathrm{H}_{2} \mathrm{O}$ & \\
\hline Sodium Hydroxide & $\mathrm{NaOH}$ & \\
\hline Sodium Nitrate & $\mathrm{NaNO}_{3}$ & \\
\hline Sodium Nitrite & $\mathrm{NaNO}_{2}$ & \\
\hline Sodium Persulfate & $\mathrm{Na}_{2} \mathrm{SO}_{2} \mathrm{O}_{8}$ & \\
\hline Sodium Phosphate & $\mathrm{Na}_{3} \mathrm{PO}_{4}$ & \\
\hline Sodium Sulfate & $\mathrm{Na}_{2} \mathrm{SO}_{4}$ & \\
\hline Soltrol-170 & $\mathrm{C}_{10} \mathrm{H}_{22}$ to $\mathrm{C}_{16} \mathrm{H}_{34}$ & Product name \\
\hline Sugar & $\mathrm{C}_{12} \mathrm{H}_{22} \mathrm{O}_{11}$ & \\
\hline Sulfamic Acid & $\mathrm{NH}_{2} \mathrm{SO}_{3} \mathrm{H}$ & \\
\hline Sulfuric Acid & $\mathrm{H}_{2} \mathrm{SO}_{4}$ & \\
\hline Tartaric Acid & $\mathrm{C}_{2} \mathrm{H}_{2}\left(\mathrm{OHCOOH}_{2}\right.$ & \\
\hline $\begin{array}{l}\text { Tetrasodium Ethylene } \\
\text { Diamine-Tetra Acetate } \\
\text { (EDTA) }\end{array}$ & $\mathrm{N}_{2} \mathrm{C}_{2} \mathrm{H}_{4}\left(\mathrm{C}_{2} \mathrm{H}_{2} \mathrm{O}_{2} \mathrm{Na}\right)_{4}$ & \\
\hline Tributyl Phosphate & & \\
\hline $\begin{array}{l}\text { Trisodium Hydroxyethyl } \\
\text { Ethylene-Diamine } \\
\text { Triacetate (HEDTA) }\end{array}$ & $\left(\mathrm{C}_{4} \mathrm{H}_{9}\right)_{3} \mathrm{PO}_{4}$ & $\mathrm{~N}_{2} \mathrm{C}_{2} \mathrm{H}_{4}\left(\mathrm{C}_{2} \mathrm{H}_{2} \mathrm{O}_{2} \mathrm{Na}\right)_{3}$ \\
$\left(\mathrm{C}_{2} \mathrm{H}_{4} \mathrm{OH}_{3}\right.$ & \\
\hline
\end{tabular}


WHC-EP-0172 Revision 1

Table 10. REDOX Plant (S Plant): Fuel Reprocessing (200 West Area) (1951-1967). (Sheet 1 of 2)

\begin{tabular}{|c|c|c|}
\hline Compound name & Formula & Comments \\
\hline Aluminum & $\mathrm{Al}$ & Fuel element constituent \\
\hline $\begin{array}{l}\text { Aluminum Nitrate } \\
\text { Nonahydrate }\end{array}$ & $\mathrm{Al}\left(\mathrm{NO}_{3}\right)_{3} \cdot 9 \mathrm{H}_{2} \mathrm{O}$ & \\
\hline $\begin{array}{l}\text { Aluminum Nitrate (Mono } \\
\text { Basic) }\end{array}$ & $\mathrm{Al}(\mathrm{OH})\left(\mathrm{NO}_{3}\right)_{2}$ & \\
\hline Ammonium Fluoride & $\mathrm{NH}_{4} \mathrm{~F}$ & \\
\hline Ammonium Nitrate & $\mathrm{NH}_{4} \mathrm{NO}_{3}$ & \\
\hline Anti-Foam 60 (GE) & Silicone Emulsion & Product name \\
\hline Boric Acid & $\mathrm{H}_{3} \mathrm{BO}_{3}$ & \\
\hline Ceric Ammonium Nitrate & $\mathrm{CeNH}_{4}\left(\mathrm{NO}_{3}\right)_{6}$ & \\
\hline Dibasic Aluminum Nitrate & $\mathrm{Al}(\mathrm{OH})_{2} \mathrm{NO}_{3}$ & \\
\hline Ferrous Ammonium Sulfate & $\mathrm{Fe}\left(\mathrm{NH}_{4}\right)_{2}\left(\mathrm{SO}_{4}\right)_{2} \cdot 6 \mathrm{H}_{2} \mathrm{O}$ & \\
\hline Ferrous Sulfamate & $\mathrm{Fe}\left(\mathrm{SO}_{3} \mathrm{NH}_{2}\right)_{2}$ & \\
\hline Hydrazine & $\mathrm{H}_{2} \mathrm{NNH}_{2} \cdot \mathrm{H}_{2} \mathrm{O}$ & \\
\hline Iron & $\mathrm{Fe}$ & Fuel element constituent \\
\hline Mercuric Nitrate & $\mathrm{Hg}\left(\mathrm{NO}_{3}\right)_{2} \cdot \mathrm{H}_{2} \mathrm{O}$ & \\
\hline Methyl Isobutyl Ketone & $\mathrm{CH}_{3} \mathrm{COC}_{4} \mathrm{H}_{9}$ & \\
\hline Mistron & $\mathrm{MgSiO}_{3}$ & Product name \\
\hline Nitric Acid & $\mathrm{HNO}_{3}$ & \\
\hline $\begin{array}{l}\text { Normal Paraffin } \\
\text { Hydrocarbon }\end{array}$ & $\mathrm{C}_{10} \mathrm{H}_{22}$ to $\mathrm{C}_{14} \mathrm{H}_{30}$ & \\
\hline Oxalic Acid & $\mathrm{HO}_{2} \mathrm{CCO}_{2} \mathrm{H} \cdot 2 \mathrm{H}_{2} \mathrm{O}$ & \\
\hline Periodic Acid & $\mathrm{HIO}_{4}$ & \\
\hline Potassium Fluoride & $\mathrm{KF}$ & \\
\hline Potassium Permanganate & $\mathrm{KMnO}_{4}$ & \\
\hline Silicon & $\mathrm{Si}$ & Fuel element constituent \\
\hline Silver Nitrate & $\mathrm{Ag}\left(\mathrm{NO}_{3}\right)_{2}$ & \\
\hline Sodium Bismuthate & $\mathrm{NaBiO}_{3}$ & \\
\hline Sodium Carbonate & $\mathrm{Na}_{2} \mathrm{CO}_{3}$ & \\
\hline Sodium Dichromate & $\mathrm{Na}_{2} \mathrm{Cr}_{2} \mathrm{O}_{7} \cdot 2 \mathrm{H}_{2} \mathrm{O}$ & \\
\hline
\end{tabular}


WHC-EP-0172 Revision 1

Table 10. REDOX Plant (S Plant): Fuel Reprocessing (200 West Area) (1951-1967). (Sheet 2 of 2)

\begin{tabular}{|l|l|l|}
\hline \multicolumn{1}{|c|}{ Compound name } & \multicolumn{1}{c|}{ Formula } & Comments \\
\hline Sodium Fluoride & $\mathrm{NaF}$ & \\
\hline Sodium Hydroxide & $\mathrm{NaOH}$ & \\
\hline Sodium Nitrate & $\mathrm{NaNO}_{3}$ & \\
\hline Sodium Nitrite & $\mathrm{NaNO}_{2}$ & \\
\hline Sulfamic Acid & $\mathrm{NH}_{2} \mathrm{SO}_{3} \mathrm{H}$ & \\
\hline Sulfuric Acid & $\mathrm{H}_{2} \mathrm{SO}_{4}$ & \\
\hline Tributyl Phosphate & $\left(\mathrm{C}_{4} \mathrm{H}_{9}\right)_{3} \mathrm{PO}_{4}$ & \\
\hline Zirconium & $\mathrm{Zr}$ & Fuel element constituent \\
\hline
\end{tabular}


Table 11. REDOX Plant (S Plant): Analytical Laboratory (200 West Area) (1951-1976). (Sheet 1 of 2)

\begin{tabular}{|c|c|c|}
\hline Compound name & Formula & Comments \\
\hline Acetone & $\mathrm{CH}_{3} \mathrm{C}_{2} \mathrm{OH}_{3}$ & \\
\hline $\begin{array}{l}\text { Aluminum Nitrate } \\
\text { Nonahydrate }\end{array}$ & $\mathrm{Al}\left(\mathrm{NO}_{3}\right)_{3} \cdot 9 \mathrm{H}_{2} \mathrm{O}$ & \\
\hline Ammonium Hydroxide & $\mathrm{NH}_{4} \mathrm{OH}$ & \\
\hline Ammonium Oxalate & $\left(\mathrm{NH}_{4}\right)_{2} \mathrm{C}_{2} \mathrm{O}_{4} \cdot \mathrm{H}_{2} \mathrm{O}$ & \\
\hline Bromonaphthalene & $\mathrm{C}_{10} \mathrm{H}_{7} \mathrm{Br}$ & \\
\hline Ceric Sulfate & $\mathrm{Ce}\left(\mathrm{SO}_{4}\right)_{2}$ & \\
\hline $\begin{array}{l}\text { Di2-Ethyl Hexyl Phosphoric } \\
\text { Acid }\end{array}$ & $\mathrm{C}_{16} \mathrm{H}_{34} \mathrm{POOH}$ & \\
\hline Ferrous Sulfamate & $\mathrm{Fe}\left(\mathrm{SO}_{3} \mathrm{NH}_{2}\right)_{2}$ & \\
\hline Ferrous Sulfate & $\mathrm{FeSO}_{4}$ & \\
\hline Hydrazine & $\mathrm{H}_{2} \mathrm{NNH}_{2} \cdot \mathrm{H}_{2} \mathrm{O}$ & \\
\hline Hydrochloric Acid & $\mathrm{HCl}$ & \\
\hline $\begin{array}{l}\text { Hydroxylamine } \\
\text { Hydrochloride }\end{array}$ & $\mathrm{NH}_{2} \mathrm{OH} \cdot \mathrm{HCl}$ & . \\
\hline Hydroxyquinoline & $\mathrm{C}_{9} \mathrm{H}_{6} \mathrm{NOH}$ & \\
\hline Lead Nitrate & $\mathrm{Pb}\left(\mathrm{NO}_{3}\right)_{2}$ & \\
\hline Mercuric Thiocyanate & $\mathrm{Hg}(\mathrm{SCN})_{2}$ & \\
\hline Methyl Ethyl Ketone & $\mathrm{CH}_{3} \mathrm{COC}_{2} \mathrm{H}_{5}$ & \\
\hline Methyl Isobutyl Ketone & $\mathrm{CH}_{3} \mathrm{COC}_{4} \mathrm{H}_{9}$ & \\
\hline Mineral Oil & Light Hydrocarbons & \\
\hline Nitric Acid & $\mathrm{HNO}_{3}$ & \\
\hline $\begin{array}{l}\text { Normal Paraffin } \\
\text { Hydrocarbon }\end{array}$ & $\mathrm{C}_{10} \mathrm{H}_{22}$ to $\mathrm{C}_{14} \mathrm{H}_{30}$ & \\
\hline O-phenanthroline & $\mathrm{C}_{12} \mathrm{H}_{8} \mathrm{~N}_{2}$ & \\
\hline Potassium Fluoride & $\mathrm{KF}$ & \\
\hline Potassium Oxalate & $\mathrm{K}_{2} \mathrm{C}_{2} \mathrm{O}_{4}$ & \\
\hline Potassium Permanganate & $\mathrm{KMnO}_{4}$ & \\
\hline S-diphenyl Carbazide & $\left(\mathrm{C}_{6} \mathrm{H}_{5} \mathrm{NH} \cdot \mathrm{HN}\right)_{2} \mathrm{CO}$ & \\
\hline Shell Spray Base & $\mathrm{C}_{10} \mathrm{H}_{22}$ to $\mathrm{C}_{16} \mathrm{H}_{34}$ & Product name \\
\hline
\end{tabular}


WHC-EP-0172 Revision 1.

Table 11. REDOX Plant (S Plant): Analytical Laboratory (200 West Area) (1951:1976). (Sheet 2 of 2)

\begin{tabular}{|l|l|l|}
\hline \multicolumn{1}{|c|}{ Compound name } & \multicolumn{1}{|c|}{ Formula } & \multicolumn{1}{c|}{ Comments } \\
\hline Sodium Dichromate & $\mathrm{Na}_{2} \mathrm{Cr}_{2} \mathrm{O}_{7} \cdot 2 \mathrm{H}_{2} \mathrm{O}$ & \\
\hline Sodium Fluoride & $\mathrm{NaF}$ & \\
\hline Sodium Hydroxide & $\mathrm{NaOH}$ & \\
\hline Sodium Nitrite & $\mathrm{NaNO}_{2}$ & \\
\hline Sulfuric Acid & $\mathrm{H}_{2} \mathrm{SO}_{4}$ & \\
\hline Tetrabromoethane & $\left(\mathrm{CHBr}_{2}\right)_{2}$ & \\
\hline Tetraphenyl Boron & $\left(\mathrm{C}_{6} \mathrm{H}_{5}\right)_{4} \mathrm{~B}$ & \\
\hline Thenoyltrifluoroacetone & $\left(\mathrm{CH}_{3} \mathrm{SCOCH}_{2}\right.$ & \\
\hline Titanium Chloride & $\mathrm{COCF}_{3}$ & \\
\hline Tributyl Phosphate & $\mathrm{TiCl}_{4}$ & \\
\hline Tri-iso-octylamine & $\left(\mathrm{C}_{4} \mathrm{H}_{9}\right)_{3} \mathrm{PO}_{4}$ & \\
\hline Tri-n-octylamine & {$\left[\left(\mathrm{CH}_{3}\right)_{2} \mathrm{CH}_{\left.\left(\mathrm{CH}_{2}\right)_{5}\right]_{3} \mathrm{~N}}\right.$} & \\
\hline Xylene & {$\left[\mathrm{CH}_{3}\left(\mathrm{CH}_{2}\right)_{7}\right]_{3} \mathrm{~N}$} & \\
\hline Zine Amalgam & $\mathrm{C}_{6} \mathrm{H}_{4}\left(\mathrm{CH}_{3}\right)_{2}$ & \\
\hline
\end{tabular}


WHC-EP-0172 Revision 1

Table 12. T Plant: Fuel Reprocessing (200 West Area) (1944-1956).

\begin{tabular}{|l|l|l|}
\hline \multicolumn{1}{|c|}{ Compound name } & \multicolumn{1}{c|}{ Formula } & \multicolumn{1}{c|}{ Comments } \\
\hline Aluminum & $\mathrm{Al}$ & Fuel element constituent \\
\hline Ammonium Nitrate & $\mathrm{NH}_{4} \mathrm{NO}_{3}$ & \\
\hline Ammonium Fluosilicate & $\left(\mathrm{NH}_{4}\right)_{2} \mathrm{SiF}_{6}$ & \\
\hline Ammonium Sulfate & $\mathrm{NH}_{4} \mathrm{SO}_{4}$ & \\
\hline Ammonium Sulfite & $\left(\mathrm{NH}_{4}\right)_{2} \mathrm{SO}_{3}$ & \\
\hline Ceric Nitrate & $\mathrm{Ce}\left(\mathrm{NO}_{3}\right)_{4}$ & \\
\hline Ferrous Sulfate & $\mathrm{FeSO}_{4}$ & \\
\hline Hydrogen Fluoride & $\mathrm{HF}_{3}$ & \\
\hline Hydrogen Peroxide & $\mathrm{H}_{2} \mathrm{O}_{2}$ & \\
\hline Hyflo-Super-Cel & $\mathrm{Contains} \mathrm{Silica}_{3}$ & Product name \\
\hline Lanthanum Nitrate & $\left.\mathrm{La}_{3} \mathrm{NO}_{3}\right)_{3}$ & \\
\hline Nitric Acid & $\mathrm{HNO}_{3}$ & \\
\hline Oxalic Acid & $\mathrm{HO}_{2} \mathrm{CCO}_{2} \mathrm{H} \cdot 2 \mathrm{H}$ & \\
\hline Phosphoric Acid & $\mathrm{H}_{3} \mathrm{PO}_{4}$ & \\
\hline Potassium Hydroxide & $\mathrm{KOH}_{3}$ & \\
\hline Potassium Permanganate & $\mathrm{KMnO}_{4}$ & \\
\hline Silicon & $\mathrm{Si}_{3}$ & \\
\hline Sodium Bismuthate & $\mathrm{NaBiO}_{3}$ & \\
\hline Sodium Carbonate & $\mathrm{Na}_{2} \mathrm{CO}_{3}$ & \\
\hline Sodium Dichromate & $\mathrm{Na}_{2} \mathrm{Cr}_{2} \mathrm{O}_{7} \cdot 2 \mathrm{H}_{2} \mathrm{O}$ & \\
\hline Sodium Hydroxide & $\mathrm{NaOH}_{3}$ & \\
\hline Sodium Nitrate & $\mathrm{NaNO}_{3}$ & \\
\hline Sodium Nitrite & $\mathrm{NaNO}_{2}$ & \\
\hline Sulfuric Acid & $\mathrm{H}_{2} \mathrm{SO}_{4}$ & \\
\hline Trichloroethane & $\mathrm{CH}_{3} \mathrm{CHCl}_{3}$ & \\
\hline Zirconyl Nitrate & \\
\hline
\end{tabular}


WHC-EP-0172 Revision 1

Table 13. T Plant: In-Plant Scavenging (200 West Area) (1955).

\begin{tabular}{|l|l|l|}
\hline \multicolumn{1}{|c|}{ Compound name } & \multicolumn{1}{|c|}{ Formula } & \multicolumn{1}{c|}{ Comments } \\
\hline Potassium Ferrocyanide & $\mathrm{K}_{4} \mathrm{Fe}(\mathrm{CN})_{6}$ & \\
\hline Nickel Sulfate & $\mathrm{NiSO}_{4}$ & \\
\hline Sodium Hydroxide & $\mathrm{NaOH}$ & \\
\hline
\end{tabular}


WHC-EP-0172 Revision 1

Table 14. 221-T Plant: Containment Systems Experiment (200 West Area) (1967-1972).

\begin{tabular}{|l|l|l|}
\hline \multicolumn{1}{|c|}{ Compound name } & \multicolumn{1}{|c|}{ Formula } & \multicolumn{1}{c|}{ Comments } \\
\hline Boric Acid & $\mathrm{H}_{3} \mathrm{BO}_{3}$ & \\
\hline Sodium Hydroxide & $\mathrm{NaOH}$ & \\
\hline Sodium Thiosulfate & $\mathrm{Na}_{2} \mathrm{~S}_{2} \mathrm{O}_{3}$ & \\
\hline
\end{tabular}


WHC-EP-0172 Revision 1

Table 15. T Plant: Containment Systems Test Facility (200 West Area) (1977).

\begin{tabular}{|l|l|l|}
\hline \multicolumn{1}{|c|}{ Compound name } & \multicolumn{1}{|c|}{ Formula } & Comments \\
\hline Sodium & $\mathrm{Na}$ & \\
\hline
\end{tabular}


Table 16. 221-T Plant: Equipment Decontamination (200 West Area) (1964-1980). (Sheet 1 of 3)

\begin{tabular}{|c|c|c|}
\hline Compound name & Formula & Comments \\
\hline Acetone & $\mathrm{CH}_{3} \mathrm{C}_{2} \mathrm{OH}_{3}$ & \\
\hline $\begin{array}{l}\text { Aluminum Nitrate } \\
\text { Nonahydrate }\end{array}$ & $\mathrm{Al}\left(\mathrm{NO}_{3}\right)_{3} \cdot 9 \mathrm{H}_{2} \mathrm{O}$ & \\
\hline Clayton Kerful Cleaner & $\mathrm{NaOH}$ & Product name \\
\hline Diversy Chemical 159 & Unknown & Product name \\
\hline Hydrochloric Acid & $\mathrm{HCl}$ & \\
\hline Immunol 1468-2 & Unknown & Product name \\
\hline Jasco Paint Stripper & $\begin{array}{l}\text { Methylene Chloride, } \\
\mathrm{CH}_{3} \mathrm{OH}\end{array}$ & Product name \\
\hline Kelite 25E & Unknown & Product name \\
\hline Keraff & Unknown & Product name \\
\hline Kerosene & $\mathrm{C}_{10} \mathrm{H}_{22}$ to $\mathrm{C}_{16} \mathrm{H}_{34}$ & \\
\hline Kleeno Bowl & $\mathrm{HCl}, \mathrm{NH}_{4} \mathrm{Cl}$ & Product name \\
\hline Nitric Acid & $\mathrm{HNO}_{3}$ & \\
\hline Oakite Clear Guard & Unknown & Product name \\
\hline Oakite LSD & $\mathrm{NaOH}$ & Product name \\
\hline Oakite Rust Stripper & Unknown & Product name \\
\hline Oakite Swiff & Trichloroethane & Product name \\
\hline Orvus $\mathrm{K}$ & Unknown & Product name \\
\hline Pace-S-Teen & Unknown & Product name \\
\hline Penvert 192 & Unknown & Product name \\
\hline Peroklean & Unknown & Product name \\
\hline Phosphoric Acid & $\mathrm{H}_{3} \mathrm{PO}_{4}$ & \\
\hline Potassium Fluoride & $\mathbf{K F}$ & \\
\hline Potassium Permanganate & $\mathrm{KMnO}_{4}$ & \\
\hline Saf-tee Solvent F.O. 128 & Unknown & Product name \\
\hline Sodium Bisulfate & $\mathrm{NaHSO}_{4} \cdot \mathrm{H}_{2} \mathrm{O}$ & \\
\hline Sodium Hydroxide & $\mathrm{NaOH}$ & \\
\hline Sodium Nitrite & $\mathrm{NaNO}_{2}$ & \\
\hline
\end{tabular}


Table 16. 221-T Plant: Equipment Decontamination (200 West Area) (1964-1980). (Sheet 2 of 3)

\begin{tabular}{|c|c|c|}
\hline Compound name & Formula & Comments \\
\hline Spartan DC 13 & $\begin{array}{l}\text { No Hazardous } \\
\text { Compounds }\end{array}$ & Product name \\
\hline Sulfuric Acid & $\mathrm{H}_{2} \mathrm{SO}_{4}$ & \\
\hline Tide (Detergent) & $\begin{array}{l}\text { Sodium Silicate } \\
\text { Compounds }\end{array}$ & Product name \\
\hline Trichloroethane & $\mathrm{CH}_{3} \mathrm{CCl}_{3}$ & \\
\hline Turco EPO Strip & Unknown & Product name \\
\hline Turco EPO Strip NP & Unknown & Product name \\
\hline Turco (Fabrifilm) & $\begin{array}{l}\text { Toluene,Butanol, } \\
\text { Isopropanol,Acetone }\end{array}$ & Product name \\
\hline Turco 2822 & $\begin{array}{l}\text { Methylene Chloride, } \\
\text { Acetic Acid }\end{array}$ & Product name \\
\hline Turco 2844 & Unknown & Product name \\
\hline Turco 4306 B, C, \& D & $\mathrm{NaHSO}_{4}, \mathrm{NH}_{2} \mathrm{SO}_{3} \mathrm{H}$ & Product name \\
\hline Turco 4358-4A & Unknown & Product name \\
\hline Turco 4501 A & $\begin{array}{l}\text { KOH,Hydroxy- } \\
\text { diamine Compounds }\end{array}$ & \\
\hline Turco 4502D & $\begin{array}{l}\mathrm{KOH}, \mathrm{K}_{2} \mathrm{CrO}_{4}, \\
\mathrm{KMnO}_{4}\end{array}$ & Product name \\
\hline Turco 4512 A & $\mathrm{H}_{3} \mathrm{PO}_{4}$ & Product name \\
\hline Turco 4518 & $\begin{array}{l}\mathrm{HO}_{2} \mathrm{CCO}_{2} \mathrm{H} \cdot 2 \mathrm{H}_{2} \mathrm{O}, \\
\text { Sodium Dodecyl } \\
\text { Benzene Sulfonate }\end{array}$ & Product name \\
\hline Turco 4521 & $\begin{array}{l}\left(\mathrm{NH}_{4}\right)_{2} \mathrm{C}_{2} \mathrm{O}_{4}, \\
\mathrm{HO}_{2} \mathrm{CCO}_{2} \mathrm{H} \cdot 2 \mathrm{H}_{2} \mathrm{O}, \\
\text { Sodium Dodecyl } \\
\text { Benzene Sulfonate }\end{array}$ & Product name \\
\hline Turco $4605-8$ & Unknown & Product name \\
\hline Turco 4669 & Unknown & Product name \\
\hline Turco 4715 & Unknown & Product name \\
\hline Turco 4738 (Thin) & Unknown & Product name \\
\hline
\end{tabular}


Table 16. 221-T Plant: Equipment Decontamination (200 West Area) (1964-1980). (Sheet 3 of 3)

\begin{tabular}{|l|l|l|}
\hline \multicolumn{1}{|c|}{ Compound name } & \multicolumn{1}{|c|}{ Formula } & \multicolumn{1}{c|}{ Comments } \\
\hline Turco T-5561 & $\begin{array}{l}\text { 2-Butoxyethanol, } \\
\text { Dioctyl Phtalate, } \\
\text { Morpholine, Mineral } \\
\text { Oil }\end{array}$ & Product name \\
\hline Turco T-5589 & Isopropanol,NH ${ }_{4} \mathrm{OH}$ & Product name \\
\hline $\begin{array}{l}\text { Turco Alkaline (Rust } \\
\text { Remover) }\end{array}$ & NaOH,Kerosene & Product name \\
\hline Turco Deseal Zit 2 & $\begin{array}{l}\text { Methylene Chloride, } \\
\text { Acidic Acid }\end{array}$ & Product name \\
\hline Turco Plaudit & $\begin{array}{l}\text { No Hazardous } \\
\text { Compounds }\end{array}$ & Product name \\
\hline West Lode Degreaser & $\begin{array}{l}\text { Aromatic } \\
\text { Compounds }\end{array}$ & Product name \\
\hline Wyandotte Kelvar & Unknown & Product name \\
\hline Wyandotte MF & Unknown & Product name \\
\hline Wyandotte P1075 & Unknown & Product name \\
\hline Wyandotte 1112 & Unknown & Product name \\
\hline
\end{tabular}


WHC-EP-0172 Revision 1

Table 17. U Plant: $\left(\mathrm{UO}_{3}\right)$ Uranium Conversion (200 West Area) (1952-1971).

\begin{tabular}{|l|l|l|}
\hline \multicolumn{1}{|c|}{ Compound name } & \multicolumn{1}{|c|}{ Formula } & Comments \\
\hline Nitric Acid & $\mathrm{HNO}_{3}$ & \\
\hline Potassium Permanganate & $\mathrm{KMnO}_{4}$ & \\
\hline Sulfuric Acid & $\mathrm{H}_{2} \mathrm{SO}_{4}$ & \\
\hline
\end{tabular}


Table 18. U Plant: Uranium Recovery and In-Plant Scavenging (200 West Area) (1952-1958).

\begin{tabular}{|l|l|l|}
\hline \multicolumn{1}{|c|}{ Compound name } & \multicolumn{1}{|c|}{ Formula } & \multicolumn{1}{c|}{ Comments } \\
\hline Calcium Carbonate & $\mathrm{CaCO}_{3}$ & \\
\hline Calcium Nitrate & $\mathrm{Ca}\left(\mathrm{NO}_{3}\right)_{2}$ & \\
\hline Cobalt Sulfate & $\mathrm{CoSO}_{4} \cdot 7 \mathrm{H}_{2} \mathrm{O}$ & \\
\hline Ferrous Ammonium Sulfate & $\mathrm{Fe}\left(\mathrm{NH}_{4}\right)_{2}\left(\mathrm{SO}_{4}\right)_{2} \cdot 6 \mathrm{H}_{2} \mathrm{O}$ & \\
\hline Ferrous Sulfamate & $\mathrm{Fe}\left(\mathrm{SO}_{3} \mathrm{NH}_{2}\right)_{2}$ & \\
\hline Nickel Sulfate & $\mathrm{NiSO}_{4}$ & \\
\hline Nitric Acid & $\mathrm{HNO}_{3}$ & \\
\hline Phosphoric Acid & $\mathrm{H}_{3} \mathrm{PO}_{4}$ & \\
\hline Potassium Ferrocyanide & $\mathrm{K}_{4} \mathrm{Fe}_{2} \mathrm{CN}_{6}$ & \\
\hline Shell Spray Base & $\mathrm{C}_{10} \mathrm{H}_{22}$ to $\mathrm{C}_{16} \mathrm{H}_{34}$ & Product name \\
\hline Shell E-2342 & $\mathrm{Naphthalene}_{3}$ and & Product name \\
\hline Paraffins & \\
\hline Sodium Carbonate & $\mathrm{Na}_{2} \mathrm{CO}_{3}$ & \\
\hline Sodium Ferrocyanide & $\mathrm{Na}_{4} \mathrm{Fe}_{2}(\mathrm{CN})_{6}$ & \\
\hline Sodium Hydroxide & $\mathrm{NaOH}_{4}$ & \\
\hline Sodium Sulfate & $\mathrm{Na}_{2} \mathrm{SO}_{4}$ & \\
\hline Strontium Nitrate & $\mathrm{Sr}_{\left(\mathrm{NO}_{3}\right)_{2}}$ & \\
\hline Sulfamic Acid & $\mathrm{NH}_{2} \mathrm{SO}_{3} \mathrm{H}$ & \\
\hline Sulfuric Acid & $\mathrm{H}_{2} \mathrm{SO}_{4}$ & \\
\hline Tributyl Phosphate & $\left(\mathrm{C}_{4} \mathrm{H}_{9}\right)_{3} \mathrm{PO}$ & \\
\hline Trichloroethane & $\mathrm{CH}_{3} \mathrm{CHCl}_{3}$ & \\
\hline & & \\
\hline
\end{tabular}


WHC-EP-0172 Revision 1

Table 19. U Plant: Analytical Laboratory (200 West Area) (1952-1958).

\begin{tabular}{|c|c|c|}
\hline Compound name & Formula & Comments \\
\hline Ammmonium Fluoride & $\mathrm{NH}_{4} \mathrm{~F}$ & \\
\hline Ammonium Nitrate & $\mathrm{NH}_{4} \mathrm{NO}_{3}$ & \\
\hline Ammonium Oxalate & $\left(\mathrm{NH}_{4}\right)_{2} \mathrm{C}_{2} \mathrm{O}_{4} \cdot \mathrm{H}_{2} \mathrm{O}$ & \\
\hline Barium Nitrate & $\mathrm{Ba}\left(\mathrm{NO}_{3}\right)_{2}$ & \\
\hline Boric Acid & $\mathrm{H}_{3} \mathrm{BO}_{3}$ & \\
\hline Carbon Tetrachloride & $\mathrm{CCl}_{4}$ & \\
\hline Ceric Iodate & $\mathrm{Ce}\left(\mathrm{IO}_{3}\right)_{4}$ & . \\
\hline Chloroplatinic Acid & $\mathrm{H}_{2} \mathrm{PtCl}_{6} \cdot 6 \mathrm{H}_{2} \mathrm{O}$ & \\
\hline Chromous Sulfate & $\mathrm{CrSO}_{4} \cdot 7 \mathrm{H}_{2} \mathrm{O}$ & \\
\hline Ethanol & $\mathrm{C}_{2} \mathrm{H}_{5} \mathrm{OH}$ & \\
\hline Ethyl Ether & $\left(\mathrm{CH}_{3} \mathrm{CH}_{2}\right)_{2} \mathrm{O}$ & \\
\hline Hydrobromic Acid & $\mathrm{HBr}$ & \\
\hline Hydrochloric Acid & $\mathrm{HCl}$ & \\
\hline Hydrofluoric Acid & HF & \\
\hline Hydroiodic Acid & HI & \\
\hline Lanthanum Fluoride & $\mathrm{LaF}_{3}$ & \\
\hline Molybdate-Citrate Reagent & $\begin{array}{l}\mathrm{MoO}_{3} \cdot \mathrm{XH}_{2} \mathrm{O}+ \\
\left(\mathrm{NH}_{4}\right)_{3} \mathrm{C}_{6} \mathrm{H}_{5} \mathrm{O}_{7}\end{array}$ & \\
\hline Oxalic Acid & $\mathrm{HO}_{2} \mathrm{CCO}_{2} \mathrm{H} \cdot 2 \mathrm{H}_{2} \mathrm{O}$ & \\
\hline Phosphorous Pentoxide & $\mathrm{P}_{2} \mathrm{O}_{5}$ & \\
\hline Potassium Carbonate & $\mathrm{K}_{2} \mathrm{CO}_{3}$ & \\
\hline Potassium Fluoride & KF & \\
\hline Potassium Hydroxide & KOH & \\
\hline Potassium Permanganate & $\mathrm{KMnO}_{4}$ & \\
\hline Sodium Fluoride & $\mathrm{NaF}$ & \\
\hline Sodium Hydroxide & $\mathrm{NaOH}$ & \\
\hline Sodium Nitrate & $\mathrm{NaNO}_{3}$ & \\
\hline Sulfuric Acid & $\mathrm{H}_{2} \mathrm{SO}_{4}$ & \\
\hline
\end{tabular}


Table 20. U Plant: Equipment Decontamination (200 West Area) (1961-1963).

\begin{tabular}{|l|l|l|}
\hline \multicolumn{1}{|c|}{ Compound name } & \multicolumn{1}{c|}{ Formula } & \multicolumn{1}{c|}{ Comments } \\
\hline Clayton Kerful Cleaner & $\mathrm{NaOH}$ & Product name \\
\hline Nitric Acid & $\mathrm{HNO}_{3}$ & \\
\hline Phosphoric Acid & $\mathrm{H}_{3} \mathrm{PO}_{4}$ & \\
\hline Potassium Hydroxide & $\mathrm{KOH}$ & \\
\hline Potassium Permanganate & $\mathrm{KMnO}_{4}$ & \\
\hline Sani-Flush & $\begin{array}{l}\mathrm{HCl}_{,} \\
\mathrm{AO}_{2} \mathrm{CCO}_{2} \mathrm{H} \cdot 2 \mathrm{H}_{2} \mathrm{O}, \\
\text { Amymoniumethyl, } \\
\text { Chloride, } \\
\text { Diethylthiourea }\end{array}$ & Product name \\
\hline Sodium Hydroxide & $\mathrm{NaOH}$ & \\
\hline Sulfuric Acid & $\mathrm{H}_{2} \mathrm{SO}$ & \\
\hline
\end{tabular}




\section{WHC-EP-0172 Revision 1}

Table 21. Plutonium Finishing Plant (Z Plant): Plutonium Conversion to Oxide (200 West Area) (1973-1976).

\begin{tabular}{|l|l|l|}
\hline \multicolumn{1}{|c|}{ Compound name } & \multicolumn{1}{|c|}{ Formula } & \multicolumn{1}{c|}{ Comments } \\
\hline Hydrogen Peroxide & $\mathrm{H}_{2} \mathrm{O}_{2}$ & \\
\hline Nitric Acid & $\mathrm{HNO}_{3}$ & \\
\hline Oxalic Acid & $\mathrm{HO}_{2} \mathrm{CCO}_{2} \mathrm{H} \cdot 2 \mathrm{H}_{2} \mathrm{O}$ & \\
\hline Potassium Permanganate & $\mathrm{KMnO}_{4}$ & \\
\hline
\end{tabular}


Table 22. Plutonium Finishing Plant (Z Plant): Plutonium Reclamation and Waste Treatment (200 West Area) (1973-1976). (Sheet 1 of 2)

\begin{tabular}{|c|c|c|}
\hline Compound name & Formula & Comments \\
\hline Aluminum & Al & Feed constituent \\
\hline $\begin{array}{l}\text { Aluminum Nitrate } \\
\text { Nonahydrate }\end{array}$ & $\mathrm{Al}\left(\mathrm{NO}_{3}\right)_{3} \cdot 9 \mathrm{H}_{2} \mathrm{O}$ & \\
\hline $\begin{array}{l}\text { Aluminum Nitrate (Mono } \\
\text { Basic) }\end{array}$ & $\mathrm{Al}(\mathrm{OH})\left(\mathrm{NO}_{3}\right)_{2}$ & \\
\hline Beryllium & $\mathrm{Be}$ & Feed constituent \\
\hline Calcium & $\mathrm{Ca}$ & Feed constituent \\
\hline Carbon Tetrachloride & $\mathrm{CCl}_{4}$ & \\
\hline Dibutyl Butyl Phosphonate & $\begin{array}{l}\mathrm{CH}_{3}\left(\mathrm{CH}_{2}\right)_{3} \mathrm{PO}_{3}\left[\mathrm{CH}_{3}\right. \\
\left.\left(\mathrm{CH}_{2}\right)_{3}\right]_{2}\end{array}$ & \\
\hline Dodecane & $\mathrm{CH}_{3}\left(\mathrm{CH}_{2}\right)_{10} \mathrm{CH}_{3}$ & Feed constituent \\
\hline Dow Anti-Foam B & Silicon Emulsion & Product name \\
\hline Gallium & Ga & Feed constituent \\
\hline Gallium Oxide & $\mathrm{Ga}_{2} \mathrm{O}_{3}$ & Feed constituent \\
\hline Hydrazine & $\mathrm{H}_{2} \mathrm{NNH}_{2} \cdot \mathrm{H}_{2} \mathrm{O}$ & \\
\hline Hydrochloric Acid & $\mathrm{HCl}$ & \\
\hline Hydrofluoric Acid & HF & \\
\hline $\begin{array}{l}\text { Hydroxylamine } \\
\text { Hydrochloride }\end{array}$ & $\mathrm{NH}_{2} \mathrm{OH} \cdot \mathrm{HCl}$ & \\
\hline Hydroxylamine Nitrate & $\mathrm{NH}_{2} \mathrm{OH} \cdot \mathrm{HNO}_{3}$ & \\
\hline Iodine & $\mathrm{I}_{2}$ & Feed constituent \\
\hline Iron & $\mathrm{Fe}$ & Scrap container \\
\hline Magnesium Oxide & MgU & Feed constituent \\
\hline Mercuric Nitrate & $\mathrm{Hg}\left(\mathrm{NO}_{3}\right)_{2} \cdot \mathrm{H}_{2} \mathrm{O}$ & \\
\hline Mistron & $\mathrm{MgSiO}_{3}$ & Product name \\
\hline Molybdenum & Mo & Feed constituent \\
\hline Nitric Acid & $\mathrm{HNO}_{3}$ & \\
\hline Oxalic Acid & $\mathrm{HO}_{2} \mathrm{CCO}_{2} \mathrm{H} \cdot 2 \mathrm{H}_{2} \mathrm{O}$ & \\
\hline Potassium Carbonate & $\mathrm{K}_{2} \mathrm{CO}_{3}$ & \\
\hline
\end{tabular}


WHC-EP-0172 Revision 1

Table 22. Plutonium Finishing Plant (Z Plant): Plutonium Reclamation and Waste Treatment (200 West Area) (1973-1976). (Sheet 2 of 2)

\begin{tabular}{|l|l|l|}
\hline \multicolumn{1}{|c|}{ Compound name } & \multicolumn{1}{c|}{ Formula } & \multicolumn{1}{c|}{ Comments } \\
\hline Sodium Carbonate & $\mathrm{Na}_{2} \mathrm{CO}_{3}$ & \\
\hline Sodium Fluoride & $\mathrm{NaF}$ & \\
\hline Sodium Hydroxide & $\mathrm{NaOH}$ & \\
\hline Sodium Nitrate & $\mathrm{NaNO}_{3}$ & \\
\hline Sulfamic Acid & $\mathrm{NH}_{2} \mathrm{SO}_{3} \mathrm{H}$ & Feed constituent \\
\hline Tributyl Phosphate & $\left(\mathrm{C}_{4} \mathrm{H}_{9}\right)_{3} \mathrm{PO}_{4}$ & \\
\hline
\end{tabular}


WHC-EP-0172 Revision 1

Table 23. Plutonium Finishing Plant (Z Plant): Analytical Laboratory (200 West Area) (1973-1976). (Sheet 1 of 2)

\begin{tabular}{|c|c|c|}
\hline Compound name & Formula & Comments \\
\hline Acetic Acid & $\mathrm{CH}_{3} \mathrm{CO}_{2} \mathrm{H}$ & \\
\hline Acetone & $\mathrm{CH}_{3} \mathrm{C}_{2} \mathrm{H}_{3} \mathrm{O}$ & \\
\hline Alizarin Yellow & $\mathrm{C}_{14} \mathrm{H}_{8} \mathrm{O}_{4}$ & \\
\hline $\begin{array}{l}\text { Aluminum Nitrate } \\
\text { Nonahydrate }\end{array}$ & $\mathrm{Al}\left(\mathrm{NO}_{3}\right)_{3} \cdot 9 \mathrm{H}_{2} \mathrm{O}$ & \\
\hline $\begin{array}{l}\text { Aluminum Nitrate (Mono } \\
\text { Basic) }\end{array}$ & $\mathrm{Al}(\mathrm{OH})\left(\mathrm{NO}_{3}\right)_{2}$ & \\
\hline Aluminum Sulfate & $\mathrm{Al}\left(\mathrm{SO}_{4}\right)_{3}$ & \\
\hline Ammonium Chloride & $\mathrm{NH}_{4} \mathrm{Cl}$ & \\
\hline Ammonium Hydroxide & $\mathrm{NH}_{4} \mathrm{OH}$ & \\
\hline Ammonium Oxalate & $\left(\mathrm{NH}_{4}\right)_{2} \mathrm{C}_{2} \mathrm{O}_{4}$ & \\
\hline Ammonium Sulfate & $\left(\mathrm{NH}_{4}\right)_{2} \mathrm{SO}_{4}$ & \\
\hline Arsenazo III & Arsenic compounds & Product name \\
\hline Boric Acid & $\mathrm{H}_{3} \mathrm{BO}_{3}$ & \\
\hline Bromocresol Purple & $\mathrm{C}_{7} \mathrm{H}_{6} \mathrm{OHBr}$ & \\
\hline Carbon Tetrachloride & $\mathrm{CCl}_{4}$ & \\
\hline Ceric Ammonium Nitrate & $\mathrm{Ce}\left(\mathrm{NH}_{4}\right)_{2}\left(\mathrm{NO}_{3}\right)_{6}$ & \\
\hline Dibutyl Phosphate & $\left(\mathrm{n}-\mathrm{C}_{4} \mathrm{H}_{9}\right)_{2} \mathrm{HPO}_{4}$ & \\
\hline Ferric Ammonium Sulfate & $\mathrm{FeNH}_{4} \mathrm{SO}_{4}$ & \\
\hline Ferric Nitrate & $\mathrm{Fe}\left(\mathrm{NO}_{3}\right)_{3} \cdot 6 \mathrm{H}_{2} \mathrm{O}$ & \\
\hline Ferrous Ammonium Sulfate & 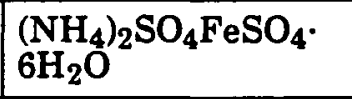 & \\
\hline Ferrous Sulfamate & $\mathrm{Fe}\left(\mathrm{SO}_{3} \mathrm{NH}_{2}\right)_{2}$ & \\
\hline Hydrazine & $\mathrm{N}_{2} \mathrm{H}_{4} \cdot \mathrm{H}_{2} \mathrm{O}$ & \\
\hline Hydrobromic Acid & $\mathrm{HBr}$ & \\
\hline Hydrochloric Acid & $\mathrm{HCl}$ & \\
\hline Hydrofluoric Acid & $\mathrm{HF}$ & \\
\hline Hydrogen Peroxide & $\mathrm{H}_{2} \mathrm{O}_{2}$ & \\
\hline Hydroiodic Acid & $\mathrm{HI}$ & \\
\hline $\begin{array}{l}\text { Hydroxylamine } \\
\text { Hydrochloride }\end{array}$ & $\mathrm{NH}_{2} \mathrm{OH} \cdot \mathrm{HCl}$ & \\
\hline
\end{tabular}


Table 23. Plutonium Finishing Plant (Z Plant): Analytical Laboratory (200 West Area) (1973-1976). (Sheet 2 of 2)

\begin{tabular}{|l|l|l|}
\hline \multicolumn{1}{|c|}{ Compound name } & \multicolumn{1}{c|}{ Formula } & Comments \\
\hline Hydroxylamine Nitrate & $\mathrm{NH}_{2} \mathrm{OH} \cdot \mathrm{HNO}_{3}$ & \\
\hline Methanol & $\mathrm{CH}_{3} \mathrm{OH}$ & \\
\hline Napthylamine & $\mathrm{C}_{10} \mathrm{H}_{9} \mathrm{~N}$ & \\
\hline Nitric Acid & $\mathrm{HNO}_{3}$ & \\
\hline Oxalic Acid & $\mathrm{HO}_{2} \mathrm{CCO}_{2}{\mathrm{H} \cdot 2 \mathrm{H}_{2} \mathrm{O}}$ & \\
\hline Phosphoric Acid & $\mathrm{H}_{3} \mathrm{PO}_{4}$ & \\
\hline Potassium Acetate & $\mathrm{KC}_{2} \mathrm{H}_{3} \mathrm{O}_{2}$ & \\
\hline Potassium Dichromate & $\mathrm{K}_{2} \mathrm{CrO}_{7}$ & \\
\hline Potassium Iodate & $\mathrm{KIO}_{3}$ & \\
\hline Potassium Permanganate & $\mathrm{KMnO}_{4}$ & \\
\hline Silver Oxide & $\mathrm{AgO}_{2}$ & \\
\hline Sodium Bisulfate & $\mathrm{NaHSO}_{4}$ & \\
\hline Sodium Carbonate & $\mathrm{Na}_{2} \mathrm{CO}_{3}$ & \\
\hline Sodium Fluoride & $\mathrm{NaF}$ & \\
\hline Sodium Hydroxide & $\mathrm{NaOH}$ & \\
\hline Sodium Nitrate & $\mathrm{NaNO}_{3}$ & \\
\hline Sodium Nitrite & $\mathrm{NaNO}_{2}$ & \\
\hline Sodium Oxalate & $\mathrm{Na}_{2} \mathrm{C}_{2} \mathrm{O}_{4}$ & \\
\hline Sodium Tartrate & $\mathrm{Na}_{2} \mathrm{C}_{2} \mathrm{H}_{4} \mathrm{O}_{6} \cdot 2 \mathrm{H}_{2} \mathrm{O}$ & \\
\hline Sulfamic Acid & $\mathrm{NH}_{2} \mathrm{SO}_{3} \mathrm{H}$ & \\
\hline Sulfonic Acid (chloro) & $\mathrm{ClHSO}_{3}$ & \\
\hline Sulfuric Acid & $\mathrm{H}_{2} \mathrm{SO}_{4}$ & \\
\hline Thenoyltrifluoracetone & $\left(\mathrm{CH}_{3} \mathrm{SCOCH}_{2}\right.$ & \\
\hline Thymolphthalein & $\mathrm{COCF}_{2} \mathrm{H}_{30} \mathrm{O}_{4}$ & \\
\hline Toluene & $\mathrm{C}_{6} \mathrm{H}_{5} \mathrm{CH}_{3}$ & \\
\hline Tributyl Phosphate & $\left(\mathrm{C}_{4} \mathrm{H}_{9}\right)_{3} \mathrm{PO}_{4}$ & \\
\hline Tri-Iso-Octylamine & $\mathrm{C}_{24} \mathrm{H}_{51} \mathrm{~N}$ & \\
\hline Tris (hydroxymethyl) Amino & $\left(\mathrm{CH}_{2} \mathrm{OH}_{3} \mathrm{CNH}_{2}\right.$ & \\
\hline Methane & $\mathrm{C}_{6} \mathrm{H}_{4}\left(\mathrm{CH}_{3}\right)_{2}$ & \\
\hline Xylene & & \\
\hline
\end{tabular}


Table 24. Tank Farms (200 Areas): Equipment Decontamination and Waste Evaporation/Solidification (1944-1980).

\begin{tabular}{|c|c|c|}
\hline Compound name & Formula & Comments \\
\hline Acetic Acid & $\mathrm{CH}_{3} \mathrm{CO}_{2} \mathrm{H}$ & \\
\hline Anti-Foam 60 & Unkṇown & Product name \\
\hline Citric Acid & $\mathrm{C}_{3} \mathrm{H}_{4} \mathrm{OH}(\mathrm{COOH})_{3}$ & \\
\hline Diatomateous Earth & $\mathrm{SiO}_{2}$ & \\
\hline Duolite ARC-359 (IX Resin) & $\mathrm{Al}_{2}\left(\mathrm{SiO}_{5}\right)_{2}(\mathrm{OH})_{2}$ & Product name \\
\hline Nitric Acid & $\mathrm{HNO}_{3}$ & \\
\hline Oxalic Acid & $\mathrm{HO}_{2} \mathrm{CCO}_{2} \mathrm{H} \cdot 2 \mathrm{H}_{2} \mathrm{O}$ & \\
\hline $\begin{array}{l}\text { Pentasodium Diethylene } \\
\text { Triamine Penta Acetate } \\
\text { (DTPA) }\end{array}$ & $\mathrm{N}_{3}\left(\mathrm{CH}_{2}\right)_{4}(\mathrm{COONa})_{5}$ & \\
\hline Potassium Permanganate & $\mathrm{KMnO}_{4}$ & \\
\hline Portland Cement & Calcium compounds & \\
\hline Sodium Carbonate & $\mathrm{Na}_{2} \mathrm{CO}_{3}$ & \\
\hline Sodium Hydroxide & $\mathrm{NaOH}$ & \\
\hline Sodium Nitrate & $\mathrm{NaNO}_{3}$ & \\
\hline Sodium Nitrite & $\mathrm{NaNO}_{2}$ & \\
\hline Spic and Span (Detergent) & $\begin{array}{l}\mathrm{Na}_{3} \mathrm{PO}_{4}, \mathrm{Na}_{2} \mathrm{SO}_{4}, \\
\mathrm{Na} \text { Silicate }\end{array}$ & Product name \\
\hline Tartaric Acid & $\mathrm{C}_{2} \mathrm{H}_{2}(\mathrm{OHCOOH})_{2}$ & \\
\hline Tide (Detergent) & $\begin{array}{l}\mathrm{Na}_{3} \mathrm{PO}_{4}, \mathrm{Na}_{2} \mathrm{CO}_{3}, \\
\mathrm{Na} \text { Silicate }\end{array}$ & Product name \\
\hline Turco $4502 \mathrm{D}$ & $\begin{array}{l}\mathrm{KOH}, \mathrm{K}_{2} \mathrm{CrO}_{4}, \\
\mathrm{KMnO}_{4}\end{array}$ & Product name \\
\hline Turco 4518 & $\begin{array}{l}\mathrm{HO}_{2} \mathrm{CCO}_{2} \mathrm{H} \cdot 2 \mathrm{H}_{2} \mathrm{O} \\
\text { Sodium Dodecyl } \\
\text { Benzene Sulfonate }\end{array}$ & Product name \\
\hline Turco Deseal Zit 2 & $\begin{array}{l}\text { Methylene Chloride, } \\
\text { Acetic Acid }\end{array}$ & Product name \\
\hline Zelon 900 (IX Resin) & $\mathrm{Al}_{2}\left(\mathrm{SiO}_{5}\right)_{2}(\mathrm{OH})_{2}$ & Product name \\
\hline
\end{tabular}


WHC-EP-0172 Revision 1

Table 25. Tank Farms (200 Areas): Waste Scavenging and Sludge Sluicing/Dissolution (1952-1976).

\begin{tabular}{|l|l|l|}
\hline \multicolumn{1}{|c|}{ Compound name } & \multicolumn{1}{c|}{ Formula } & \multicolumn{1}{c|}{ Comments } \\
\hline $\begin{array}{l}\text { American Cyanamid S 4058 } \\
\text { Floc }\end{array}$ & Hydrocarbon & Product name \\
\hline Calcium Carbonate & $\mathrm{CaCO}_{3}$ & \\
\hline Calcium Nitrate & $\mathrm{Ca}\left(\mathrm{NO}_{3}\right)_{2}$ & \\
\hline Dow N 20 Floc & Unknown & Product name \\
\hline Dow XD 7889 Floc & Unknown & Product name \\
\hline Nickel Sulfate & $\left.\mathrm{Ni}_{(\mathrm{SO}}\right)_{2}$ & \\
\hline Nitric Acid & $\mathrm{HNO}$ & \\
\hline Sodium Ferrocyanide & $\mathrm{Na}_{4} \mathrm{Fe}(\mathrm{CN})_{6}$ & \\
\hline Sodium Sulfide & $\mathrm{Na}_{2} \mathrm{~S}$ & \\
\hline
\end{tabular}


WHC-EP-0172 Revision 1

Table 26. Building 242 (200 East Area): Irradiation Rupture Prototype Loop (1959-1967).

\begin{tabular}{|l|l|l|}
\hline \multicolumn{1}{|c|}{ Compound name } & \multicolumn{1}{c|}{ Formula } & Comments \\
\hline Carbon Dioxide & $\mathrm{CO}_{2}$ & \\
\hline Citric Acid & $\mathrm{C}_{3} \mathrm{H}_{4} \mathrm{OH}(\mathrm{COOH})_{3}$ & \\
\hline Oxalic Acid & $\mathrm{HO}_{2} \mathrm{CCO}_{2} \mathrm{H} \cdot 2 \mathrm{H}_{2} \mathrm{O}$ & \\
\hline Phosphoric Acid & $\mathrm{H}_{3} \mathrm{PO}_{4}$ & \\
\hline Sodium Hydroxide & $\mathrm{NaOH}$ & \\
\hline $\begin{array}{l}\text { Trisodium Hydroxyethyl } \\
\text { Ethylene-Diamine } \\
\text { Triacetate (HEDTA) }\end{array}$ & $\begin{array}{l}\mathrm{N}_{2} \mathrm{C}_{2} \mathrm{H}_{4}\left(\mathrm{C}_{2} \mathrm{H}_{2} \mathrm{O}_{2} \mathrm{Na}\right)_{3} \\
\left(\mathrm{C}_{2} \mathrm{H}_{4} \mathrm{OH}\right)\end{array}$ & \\
\hline
\end{tabular}


The following tables list the chemicals used in the 300 Area facilities. There is uncertainty that these chemicals were routed to the SSTs.

Table 27. Building 324: Chemicál Engineering Hot Cells (1966-1976).

\begin{tabular}{|c|c|c|}
\hline Compound name & Formula & Comments \\
\hline $\begin{array}{l}\text { Aluminum Nitrate } \\
\text { Nonahydrate }\end{array}$ & $\mathrm{Al}\left(\mathrm{NO}_{3}\right)_{3} \cdot 9 \mathrm{H}_{2} \mathrm{O}$ & \\
\hline Aluminum Sulfate & $\mathrm{Al}_{2}\left(\mathrm{SO}_{4}\right)_{3}$ & \\
\hline Barium Nitrate & $\mathrm{Ba}\left(\mathrm{NO}_{3}\right)_{2}$ & \\
\hline Copper Nitrate & $\mathrm{Cu}\left(\mathrm{NO}_{3}\right)_{2}$ & \\
\hline Ferric Nitrate & $\mathrm{Fe}\left(\mathrm{NO}_{3}\right)_{3}$ & \\
\hline Molybdenum & Mo & \\
\hline Nickel Nitrate & $\mathrm{Ni}\left(\mathrm{NO}_{3}\right)_{2} \cdot 6 \mathrm{H}_{2} \mathrm{O}$ & \\
\hline Nitric Acid & $\mathrm{HNO}_{3}$ & \\
\hline Oxalic Acid & $\mathrm{HO}_{2} \mathrm{CCO}_{2} \mathrm{H} \cdot 2 \mathrm{H}_{2} \mathrm{O}$ & \\
\hline Phosphoric Acid & $\mathrm{H}_{3} \mathrm{PO}_{4}$ & \\
\hline Potassium Dichromate & $\mathrm{K}_{2} \mathrm{CrO}_{7}$ & \\
\hline Potassium Permanganate & $\mathrm{KMnO}_{4}$ & \\
\hline Silicon & $\mathrm{Si}$ & \\
\hline Silver Nitrate & $\mathrm{AgNO}_{3}$ & \\
\hline Sodium Carbonate & $\mathrm{Na}_{2} \mathrm{CO}_{3}$ & \\
\hline Sodium Hydroxide & $\mathrm{NaOH}$ & \\
\hline Sodium Nitrate & $\mathrm{NaNO}_{3}$ & \\
\hline Sodium Phosphate & $\mathrm{Na}_{3} \mathrm{PO}_{4}$ & \\
\hline Strontium Nitrate & $\mathrm{Sr}\left(\mathrm{NO}_{3}\right)_{2}$ & \\
\hline Sulfamic Acid & $\mathrm{NH}_{2} \mathrm{SO}_{3} \mathrm{H}$ & \\
\hline Turco $4501 \mathrm{~A}$ & $\begin{array}{l}\mathrm{KOH}, \mathrm{Hydoxy}- \\
\text { diamine compounds }\end{array}$ & Product name \\
\hline Turco 4502 D & $\begin{array}{l}\mathrm{KOH}, \\
\mathrm{K}_{2} \mathrm{CrO}_{4}, \mathrm{KMnO}_{4}\end{array}$ & Product name \\
\hline $\begin{array}{c}\text { Turco } 4518 \\
.\end{array}$ & $\begin{array}{l}\mathrm{HO}_{2} \mathrm{CCO}_{2} \mathrm{H} \cdot 2 \mathrm{H}_{2} \mathrm{O} \\
\text { Sodium Dodecyl } \\
\text { Bezene Sulfonate }\end{array}$ & Product name \\
\hline Turco 4521 & $\begin{array}{l}\left(\mathrm{NH}_{4}\right)_{2} \mathrm{C}_{2} \mathrm{O}_{4} \\
\mathrm{HO}_{2} \mathrm{CCO}_{2} \mathrm{H} \cdot 2 \mathrm{H}_{2} \mathrm{O}, \\
\text { Sodium Dodecyl } \\
\text { Benzene Sulfonate }\end{array}$ & Product name \\
\hline
\end{tabular}


WHC-EP-0172 Revision 1

Table 28. Building 325: Analytical Laboratory (1953-1976). (Sheet 1 of 3)

\begin{tabular}{|c|c|c|}
\hline Compound name & Formula & Comments \\
\hline Acetone & $\mathrm{CH}_{3} \mathrm{C}_{2} \mathrm{OH}_{3}$ & \\
\hline $\begin{array}{l}\text { Aluminum Nitrate } \\
\text { Nonahydrate }\end{array}$ & $\mathrm{Al}\left(\mathrm{NO}_{3}\right)_{3} \cdot 9 \mathrm{H}_{2} \mathrm{O}$ & \\
\hline Ammonia (Anhydrous) & $\mathrm{NH}_{3}$ & \\
\hline Ammonium Hydroxide & $\mathrm{NH}_{4} \mathrm{OH}$ & \\
\hline Ammonium Oxalate & $\left(\mathrm{NH}_{4}\right)_{2} \mathrm{C}_{2} \mathrm{O}_{4} \cdot \mathrm{H}_{2} \mathrm{O}$ & \\
\hline Bromocresol Purple & $\mathrm{C}_{7} \mathrm{H}_{6} \mathrm{OHBr}$ & \\
\hline Calcium Carbonate & $\mathrm{CaCO}_{3}$ & \\
\hline Calcium Chloride & $\mathrm{CaCl}_{2}$ & \\
\hline Carbon Tetrachloride & $\mathrm{CCl}_{4}$ & \\
\hline Ceric Sulfate & $\mathrm{Ce}\left(\mathrm{SO}_{4}\right)_{2}$ & \\
\hline Cesium Nitrate & $\mathrm{CsNO}_{3}$ & \\
\hline Ethanol & $\mathrm{C}_{2} \mathrm{H}_{5} \mathrm{OH}$ & \\
\hline Ferrous Ammonium Sulfate & $\mathrm{Fe}\left(\mathrm{NH}_{4}\right)_{2}\left(\mathrm{SO}_{4}\right)_{2} \cdot 6 \mathrm{H}_{2} \mathrm{O}$ & \\
\hline Hydrazine & $\mathrm{H}_{2} \mathrm{NNH}_{2} \cdot \mathrm{H}_{2} \mathrm{O}$ & \\
\hline Hydrochloric Acid & $\mathrm{HCl}$ & \\
\hline Hydrofluoric Acid & $\mathrm{HF}$ & \\
\hline Hydrogen Peroxide & $\mathrm{H}_{2} \mathrm{O}_{2}$ & \\
\hline Hydroiodic Acid & $\mathrm{HI}$ & \\
\hline $\begin{array}{l}\text { Hydroxylamine } \\
\text { Hydrochloride }\end{array}$ & $\mathrm{NH}_{2} \mathrm{OH} \cdot \mathrm{HCl}$ & \\
\hline Iodine & $\mathrm{I}_{3}$ & \\
\hline Iron & $\mathrm{Fe}$ & \\
\hline Kerosene & $\mathrm{C}_{10} \mathrm{H}_{22}$ to $\mathrm{C}_{16} \mathrm{H}_{34}$ & \\
\hline Magnesium Oxide & $\mathrm{MgO}$ & \\
\hline Mercury & $\mathrm{Hg}$ & \\
\hline Mercuric Nitrate & $\mathrm{Hg}\left(\mathrm{NO}_{3}\right)_{2} \cdot \mathrm{H}_{2} \mathrm{O}$ & \\
\hline Methanol & $\mathrm{CH}_{3} \mathrm{OH}$ & \\
\hline Methyl Ethyl Ketone & $\mathrm{CH}_{3} \mathrm{COC}_{2} \mathrm{H}_{5}$ & \\
\hline Methyl Isobutyl Ketone & $\mathrm{CH}_{3} \mathrm{COC}_{4} \mathrm{H}_{9}$ & \\
\hline
\end{tabular}


Table 28. Building 325: Analytical Laboratory (1953-1976). (Sheet 2 of 3 )

\begin{tabular}{|c|c|c|}
\hline Compound name & Formula & Comments \\
\hline Methyi Lactic Acid & $\mathrm{CH}_{3} \mathrm{CH}(\mathrm{OH}) \mathrm{CO}_{2} \mathrm{CH}_{3}$ & \\
\hline Nitric Acid & $\mathrm{HNO}_{3}$ & \\
\hline Nitrous Acid & $\mathrm{HNO}_{2}$ & \\
\hline Oxalic Acid & $\mathrm{HO}_{2} \mathrm{CCO}_{2} \mathrm{H} \cdot 2 \mathrm{H}_{2} \mathrm{O}$ & \\
\hline Perchloric Acid & $\mathrm{HClO}_{4}$ & \\
\hline Periodic Acid & $\mathrm{IO}_{4}$ & \\
\hline Phosphoric Acid & $\mathrm{H}_{3} \mathrm{PO}_{4}$ & \\
\hline Potassium Hydroxide & $\mathrm{KOH}$ & \\
\hline Potassium Iodate & $\mathrm{KIO}_{3}$ & \\
\hline Potassium Permanganate & $\mathrm{KMnO}_{4}$ & \\
\hline Silver Nitrate & $\mathrm{AgNO}_{3}$ & \\
\hline Sodium Carbonate & $\mathrm{Na}_{2} \mathrm{CO}_{3}$ & \\
\hline Sodium Hydroxide & $\mathrm{NaOH}$ & \\
\hline Stainless Steel & FeNiCr & \\
\hline Strontium Nitrate & $\mathrm{Sr}\left(\mathrm{NO}_{3}\right)_{2}$ & \\
\hline Sulfuric Acid & $\mathrm{H}_{2} \mathrm{SO}_{4}$ & \\
\hline $\begin{array}{l}\text { Tetrasodium Ethylene } \\
\text { Diamine- } \\
\text { Tetra Acetate (EDTA) }\end{array}$ & $\mathrm{N}_{2} \mathrm{C}_{2} \mathrm{H}_{4}\left(\mathrm{C}_{2} \mathrm{H}_{2} \mathrm{O}_{2} \mathrm{Na}\right)_{4}$ & \\
\hline Tetraphenyl Boron & $\left(\mathrm{C}_{6} \mathrm{H}_{5}\right)_{4} \mathrm{~B}$ & \\
\hline Thenoyltrifluoroacetone & $\begin{array}{l}(\mathrm{CH})_{3} \mathrm{SCOCH}_{2} \\
\mathrm{COCF}_{3}\end{array}$ & \\
\hline Tide (Detergent) & $\begin{array}{l}\text { Sodium silicate } \\
\text { compounds }\end{array}$ & Product name \\
\hline Tributyl Phosphate & $\left(\mathrm{C}_{4} \mathrm{H}_{9}\right)_{3} \mathrm{PO}_{4}$ & \\
\hline Trichloroethane & $\mathrm{CH}_{3} \mathrm{CCl}_{3}$ & \\
\hline Tri-iso-octylamine & {$\left[\left(\mathrm{CH}_{3}\right)_{2} \mathrm{CH}\left(\mathrm{CH}_{2}\right)_{5}\right]_{3} \mathrm{~N}$} & \\
\hline Tri-n-octylamine & {$\left[\left(\mathrm{CH}_{3}\right)\left(\mathrm{CH}_{2}\right)_{7}\right]_{3} \mathrm{~N}$} & \\
\hline $\begin{array}{l}\text { Turco Alkaline (Rust } \\
\text { Remover) }\end{array}$ & $\mathrm{NaOH}$, Kerosene & Product name \\
\hline
\end{tabular}


WHC-EP-0172 Revision 1

Table 28. Building 325: Analytical Laboratory (1953-1976). (Sheet 3 of 3)

\begin{tabular}{|l|l|l|}
\hline \multicolumn{1}{|c|}{ Compound name } & \multicolumn{1}{c|}{ Formula } & \multicolumn{1}{c|}{ Comments } \\
\hline Turco Deseal Zit 2 & $\begin{array}{l}\text { Methylene Chloride, } \\
\text { Acetic Acid }\end{array}$ & Product name \\
\hline Turco Plaudit & $\begin{array}{l}\text { No Hazardous } \\
\text { Compounds }\end{array}$ & Product name \\
\hline Vanadium Pentoxide & $\mathrm{V}_{2} \mathrm{O}_{5}$ & \\
\hline Xylene & $\mathrm{C}_{6} \mathrm{H}_{4}\left(\mathrm{CH}_{3}\right)_{2}$ & \\
\hline Zirconyl Nitrate & $\mathrm{ZrO}\left(\mathrm{NO}_{3}\right)_{2}$ & \\
\hline
\end{tabular}


Table 29. Building 325: Westinghouse Hanford Company Development Laboratory (1970-1976). (Sheet 1 of 3)

\begin{tabular}{|c|c|c|}
\hline Compound name & Formula & Comments \\
\hline Acetic Acid & $\mathrm{CH}_{3} \mathrm{CO}_{2} \mathrm{H}$ & \\
\hline Acetone & $\mathrm{CH}_{3} \mathrm{C}_{2} \mathrm{OH}_{3}$ & \\
\hline Aluminum & $\mathrm{Al}$ & \\
\hline Ammonia (Anhydrous) & $\mathrm{NH}_{3}$ & \\
\hline Ammonium Oxalate & $\left(\mathrm{NH}_{4}\right)_{2} \mathrm{C}_{2} \mathrm{O}_{4} \cdot \mathrm{H}_{2} \mathrm{O}$ & \\
\hline Barium Nitrate & $\mathrm{Ba}\left(\mathrm{NO}_{3}\right)_{2}$ & \\
\hline Beryllium & $\mathrm{Be}$ & Fuel element constituent \\
\hline Boric Acid & $\mathrm{H}_{3} \mathrm{BO}_{3}$ & \\
\hline Calcium Carbonate & $\mathrm{CaCO}_{3}$ & \\
\hline Calcium Chloride & $\mathrm{CaCl}_{2}$ & \\
\hline Calcium Nitrate & $\mathrm{Ca}\left(\mathrm{NO}_{3}\right)_{2}$ & \\
\hline Ceric Nitrate & $\mathrm{Ce}\left(\mathrm{NO}_{3}\right)_{4}$ & \\
\hline Cerous Nitrate & $\mathrm{Ce}\left(\mathrm{NO}_{3}\right)_{3}$ & \\
\hline Cesium & $\mathrm{Cs}$ & \\
\hline Chromic Acid & $\mathrm{H}_{2} \mathrm{CrO}_{4}$ & \\
\hline Citric Acid & $\mathrm{C}_{3} \mathrm{H}_{4} \mathrm{OH}(\mathrm{COOH})_{3}$ & \\
\hline Diatomateous Earth & $\mathrm{SiO}_{2}$ & \\
\hline Ethanol & $\mathrm{CH}_{3} \mathrm{CH}_{2} \mathrm{OH}$ & \\
\hline Ferric Ammonium Sulfate & $\mathrm{Fe}_{3} \mathrm{NH}_{4}\left(\mathrm{SO}_{4}\right)_{2}$ & \\
\hline Ferric Nitrate & $\mathrm{Fe}\left(\mathrm{NO}_{3}\right)_{3} \cdot 6 \mathrm{H}_{2} \mathrm{O}$ & \\
\hline Ferric Sulfate & $\mathrm{Fe}_{2}\left(\mathrm{SO}_{4}\right)_{3}$ & \\
\hline Hydrazine & $\mathrm{H}_{2} \mathrm{NNH}_{2} \cdot \mathrm{H}_{2} \mathrm{O}$ & \\
\hline Hydrochloric Acid & $\mathrm{HCl}$ & \\
\hline Iodine & $\mathrm{I}_{2}$ & \\
\hline Iron & $\mathrm{Fe}$ & \\
\hline Isopropyl Alcohol & $\mathrm{C}_{3} \mathrm{H}_{7} \mathrm{OH}$ & \\
\hline $\begin{array}{l}\text { Lanthanum-Neodynium } \\
\text { Nitrate }\end{array}$ & $\mathrm{La}\left(\mathrm{NO}_{3}\right)_{3}-\mathrm{Nd}\left(\mathrm{NO}_{3}\right)_{3}$ & \\
\hline Lead Nitrate & $\mathrm{Pb}\left(\mathrm{NO}_{3}\right)_{2}$ & \\
\hline Magnesium Nitrate & $\mathrm{Mg}\left(\mathrm{NO}_{3}\right)_{2}$ & \\
\hline
\end{tabular}


WHC-EP-0172 Revision 1

Table 29. Building 325: Westinghouse Hanford Company Development Laboratory (1970-1976). (Sheet 2 of 3)

\begin{tabular}{|c|c|c|}
\hline Compound name & Formula & Comments \\
\hline Mercuric Nitrate & $\mathrm{Hg}\left(\mathrm{NO}_{3}\right)_{2} \cdot \mathrm{H}_{2} \mathrm{O}$ & \\
\hline Methanol & $\mathrm{CH}_{3} \mathrm{OH}$ & \\
\hline Mineral Oil & Light Hydrocarbons & \\
\hline Molybdenum & Mo & \\
\hline Nitric Acid & $\mathrm{HNO}_{3}$ & \\
\hline Nitrous Acid & $\mathrm{HNO}_{2}$ & \\
\hline $\begin{array}{l}\text { Normal Paraffin } \\
\text { Hydrocarbon }\end{array}$ & $\mathrm{C}_{10} \mathrm{H}_{22}$ to $\mathrm{C}_{14} \mathrm{H}_{30}$ & \\
\hline Phosphoric Acid. & $\mathrm{H}_{3} \mathrm{PO}_{4}$ & \\
\hline Phosphorous Pentoxide & $\mathrm{P}_{2} \mathrm{O}_{5}$ & \\
\hline Potassium Bicarbonate & $\mathrm{KHCO}_{3}$ & \\
\hline Potassium Carbonate & $\mathrm{K}_{2} \mathrm{CO}_{3}$ & \\
\hline Potassium Dichromate & $\mathrm{K}_{2} \mathrm{CrO}_{7}$ & \\
\hline Portland Cement & Calcium compounds & \\
\hline Potassium Permanganate & $\mathrm{KMnO}_{4}$ & \\
\hline Silver Nitrate & $\mathrm{AgNO}_{3}$ & \\
\hline Sodium Bicarbonate & $\mathrm{NaHCO}_{3}$ & \\
\hline Sodium Bisulfate & $\mathrm{NaHSO}_{4} \cdot \mathrm{H}_{2} \mathrm{O}$ & \\
\hline Sodium Carbonate & $\mathrm{Na}_{2} \mathrm{CO}_{3}$ & \\
\hline Sodium Dichromate & $\mathrm{Na}_{2} \mathrm{Cr}_{2} \mathrm{O}_{7} \cdot \mathrm{H}_{2} \mathrm{O}$ & \\
\hline Sodium Hydroxide & $\mathrm{NaOH}$ & \\
\hline Sudium Hypochlorite & $\mathrm{NaOCl}$ & \\
\hline Sndium Iodide & $\mathrm{NaI}$ & \\
\hline Sodium Nitrate & $\mathrm{NaNO}_{3}$ & \\
\hline Sodium Nitrite & $\mathrm{NaNO}_{2}$ & \\
\hline Sodium Oxalate & $\mathrm{Na}_{2} \mathrm{C}_{2} \mathrm{O}_{4}$ & \\
\hline Sodium Phosphate & $\mathrm{Na}_{3} \mathrm{PO}_{4}$ & \\
\hline Sodium Silicate & $\mathrm{Na}_{2} \mathrm{O} \cdot \mathrm{SiO}_{2}$ & \\
\hline Sodium Sulfate & $\mathrm{Na}_{2} \mathrm{SO}_{4}$ & \\
\hline Stainless Steel & $\mathrm{FeNiCr}$ & \\
\hline
\end{tabular}


WHC-EP-0172 Revision 1

Table 29. Building 325: Westinghouse Hanford Company Development Laboratory (1970-1976). (Sheet 3 of 3)

\begin{tabular}{|c|c|c|}
\hline Compound name & Formula & Comments \\
\hline Strontium Nitrate & $\mathrm{Sr}\left(\mathrm{NO}_{3}\right)_{2}$ & \\
\hline Sugar & $\mathrm{C}_{12} \mathrm{H}_{22} \mathrm{O}_{11}$ & \\
\hline Sulfuric Acid & $\mathrm{H}_{2} \mathrm{SO}_{4}$ & \\
\hline $\begin{array}{l}\text { Tetrasodium Ethylene } \\
\text { Diamine- } \\
\text { TetraAcetate (EDTA) }\end{array}$ & $\mathrm{N}_{2} \mathrm{C}_{2} \mathrm{H}_{4}\left(\mathrm{C}_{2} \mathrm{H}_{2} \mathrm{O}_{2} \mathrm{Na}\right)_{4}$ & \\
\hline Tide (Detergent) & $\begin{array}{l}\mathrm{Na}_{3} \mathrm{PO}_{4}, \mathrm{Na}_{2} \mathrm{CO}_{3} \\
\mathrm{Na} \text { Silicate }\end{array}$ & Product name \\
\hline Tributyl Phosphate & $\left(\mathrm{C}_{4} \mathrm{H}_{9}\right)_{3} \mathrm{PO}_{4}$ & \\
\hline Turco 4502 D & $\begin{array}{l}\mathrm{KOH}, \mathrm{K}_{2} \mathrm{CrO}_{4}, \\
\mathrm{KMnO}_{4}\end{array}$ & Product name \\
\hline Turco 4518 & $\begin{array}{l}\mathrm{HO}_{2} \mathrm{CCO}_{2} \mathrm{H} \cdot 2 \mathrm{H}_{2} \mathrm{O}, \\
\text { Sodium Dodecyl } \\
\text { Benene Sulfonate }\end{array}$ & Product name \\
\hline Urea & $\mathrm{CO}\left(\mathrm{NH}_{2}\right)_{2}$ & \\
\hline Vanadium & V & \\
\hline Zine Nitrate & $\mathrm{Zn}\left(\mathrm{NO}_{3}\right)_{2}$ & \\
\hline Zirconium & $\mathbf{Z r}$ & \\
\hline
\end{tabular}


Table 30. Building 325: Pacific Northwest Laboratory Development and Production Laboratory (1955-1976). (Sheet. 1 of 2)

\begin{tabular}{|c|c|c|}
\hline Compound name & Formula & Comments \\
\hline $\begin{array}{l}\text { Aluminum Nitrate } \\
\text { Nonahydrate }\end{array}$ & $\mathrm{Al}\left(\mathrm{NO}_{3}\right)_{3} \cdot 9 \mathrm{H}_{2} \mathrm{O}$ & \\
\hline Acetone & $\mathrm{CH}_{3} \mathrm{C}_{2} \mathrm{OH}_{3}$ & \\
\hline Ammonium Hydroxide & $\mathrm{NH}_{4} \mathrm{OH}$ & \\
\hline Carbon Tetrachloride & $\mathrm{CCl}_{4}$ & \\
\hline Cesium Nitrate & $\mathrm{CsNO}_{3}$ & \\
\hline Copper Nitrate & $\mathrm{Cu}\left(\mathrm{NO}_{3}\right)_{2}$ & \\
\hline Dowex 50 (IX Resin) & $\begin{array}{l}\text { Sulfonate } \\
\text { Polystyrene Divinyl } \\
\text { Benzene }\end{array}$ & Product name \\
\hline Ethanol & $\mathrm{C}_{2} \mathrm{H}_{5} \mathrm{OH}$ & \\
\hline Ferrous Sulfamate & $\mathrm{Fe}\left(\mathrm{SO}_{3} \mathrm{NH}_{2}\right)_{2}$ & \\
\hline Hydrazine & $\mathrm{H}_{2} \mathrm{NNH}_{2} \cdot \mathrm{H}_{2} \mathrm{O}$ & \\
\hline Hydrochloric Acid & $\mathrm{HCl}$ & \\
\hline Hydrofluoric Acid & $\mathrm{HF}$ & \\
\hline Hydroxylamine Nitrate & $\mathrm{NH}_{2} \mathrm{OH} \cdot \mathrm{HNO}_{3}$ & \\
\hline Lanthanum Nitrate & $\mathrm{La}\left(\mathrm{NO}_{3}\right)_{3}$ & \\
\hline Methanol & $\mathrm{CH}_{3} \mathrm{OH}$ & \\
\hline Mercury & $\mathrm{Hg}$ & \\
\hline Nitric Acid & $\mathrm{HNO}_{3}$ & \\
\hline Nitrilotriacetic Acid (NTA) & $\mathrm{N}\left(\mathrm{CH}_{2} \mathrm{COOH}\right)_{3}$ & \\
\hline $\begin{array}{l}\text { Normal Paraffin } \\
\text { Hydrocarbon }\end{array}$ & $\mathrm{C}_{10} \mathrm{H}_{22}$ to $\mathrm{C}_{14} \mathrm{H}_{30}$ & \\
\hline Oxalic Acid & $\mathrm{HO}_{2} \mathrm{CCO}_{2} \mathrm{H} \cdot 2 \mathrm{H}_{2} \mathrm{O}$ & \\
\hline $\begin{array}{l}\text { Pentasodium Diethylene } \\
\text { Triamine PentaAcetate } \\
\text { (DTPA). }\end{array}$ & $\mathrm{N}_{3}\left(\mathrm{CH}_{2}\right)_{4}(\mathrm{COONa})_{5}$ & \\
\hline Potassium Permanganate & $\mathrm{KMnO}_{4}$ & \\
\hline Platinum & $\mathrm{Pt}$ & \\
\hline Sodium Dichromate & $\mathrm{Na}_{2} \mathrm{Cr}_{2} \mathrm{O}_{7} \cdot 2 \mathrm{H}_{2} \mathrm{O}$ & \\
\hline Sodium Fluoride & $\mathrm{NaF}$ & \\
\hline
\end{tabular}


Table 30. Building 325: Pacific Northwest Laboratory Development and Production Laboratory (1955-1976). (Sheet 2 of 2)

\begin{tabular}{|c|c|c|}
\hline Compound name & Formula & Comments \\
\hline Sodium Hydroxide & $\mathrm{NaOH}$ & \\
\hline Sodium Nitrate & $\mathrm{NaNO}_{3}$ & \\
\hline Sodium Phosphate & $\mathrm{Na}_{3} \mathrm{PO}_{4}$ & \\
\hline Sodium Sulfate & $\mathrm{Na}_{2} \mathrm{SO}_{4}$ & \\
\hline Strontium Nitrate & $\mathrm{Sr}\left(\mathrm{NO}_{3}\right)_{2}$ & \\
\hline Sulfuric Acid & $\mathrm{H}_{2} \mathrm{SO}_{4}$ & \\
\hline $\begin{array}{l}\text { Tetrasodium Ethylene } \\
\text { Diamine-Tetraacetate } \\
\text { (EDTA) }\end{array}$ & $\mathrm{N}_{2} \mathrm{C}_{3} \mathrm{H}_{4}\left(\mathrm{C}_{2} \mathrm{H}_{2} \mathrm{O}_{2} \mathrm{Na}\right)_{4}$ & \\
\hline Tributyl Phosphate & $\left(\mathrm{C}_{4} \mathrm{H}_{9}\right)_{3} \mathrm{PO}_{4}$ & \\
\hline $\begin{array}{l}\text { Trisodium Hydroxyethyl } \\
\text { Ethylene-Diamine } \\
\text { Triacetate (HEDTA) }\end{array}$ & $\begin{array}{l}\mathrm{N}_{2} \mathrm{C}_{2} \mathrm{H}_{4}\left(\mathrm{C}_{2} \mathrm{H}_{2} \mathrm{O}_{2} \mathrm{Na}\right)_{3} \\
\left(\mathrm{C}_{2} \mathrm{H}_{4} \mathrm{OH}\right)\end{array}$ & \\
\hline Turco (Fabrifilm) & $\begin{array}{l}\text { Toluene, Butanol, } \\
\text { Isopropanol, Acetone }\end{array}$ & Product name \\
\hline Turco 4502 D & $\begin{array}{l}\mathrm{KOH}, \mathrm{K}_{2} \mathrm{CrO}_{4}, \\
\mathrm{KMnO}_{4}\end{array}$ & Product name \\
\hline Turco 4518 & $\begin{array}{l}\mathrm{HO}_{2} \mathrm{CCO}_{2} \mathrm{H} \cdot 2 \mathrm{H}_{2} \mathrm{O}, \\
\text { Sodium Dodecyl } \\
\text { Benzene Sulfonate }\end{array}$ & Product name \\
\hline Yttrium Nitrate & $\mathrm{Y}\left(\mathrm{NO}_{3}\right)_{3}$ & \\
\hline Zinc Nitrate & $\mathrm{Zn}\left(\mathrm{NO}_{3}\right)_{2}$ & \\
\hline
\end{tabular}


Table 31. Building 326: Electropolishing of Irradiated Alloy for ElectronMicroscopy (1963-1976).

\begin{tabular}{|l|l|l|}
\hline \multicolumn{1}{|c|}{ Compound name } & \multicolumn{1}{c|}{ Formula } & \multicolumn{1}{c|}{ Comments } \\
\hline Acetic Acid & $\mathrm{CH}_{3} \mathrm{CO}_{2} \mathrm{H}$ & \\
\hline Aluminum & $\mathrm{Al}$ & \\
\hline Beryllium & $\mathrm{Be}$ & \\
\hline Bromine & $\mathrm{Br}_{2}$ & \\
\hline Butanol & $\mathrm{C}_{4} \mathrm{H}_{9} \mathrm{OH}$ & \\
\hline Copper & $\mathrm{Cu}$ & \\
\hline Hydrochloric Acid & $\mathrm{HCl}$ & \\
\hline Hydrofluoric Acid & $\mathrm{HF}$ & \\
\hline Methanol & $\mathrm{CH}_{3} \mathrm{OH}$ & \\
\hline Molybdenum & $\mathrm{Mo}$ & \\
\hline Niobium & $\mathrm{Nb}$ & \\
\hline Nitric Acid & $\mathrm{HNO}$ & \\
\hline Perchloric Acid & $\mathrm{HClO}$ & \\
\hline Stainless Steel & $\mathrm{FeNiCr}$ & Fuel element constituent \\
\hline Sulfuric Acid & $\mathrm{H}_{2} \mathrm{SO}_{4}$ & \\
\hline Vanadium & $\mathrm{V}$ & Fuel element constituent \\
\hline Zirconium & $\mathrm{Zr}$ & \\
\hline & & \\
\hline
\end{tabular}


Table 32. Building 326: Metallography Laboratory (1955-1976).

\begin{tabular}{|l|l|l|}
\hline \multicolumn{1}{|c|}{ Compound name } & \multicolumn{1}{c|}{ Formula } & \multicolumn{1}{c|}{ Comments } \\
\hline Aluminum & $\mathrm{Al}$ & \\
\hline Acetic Acid & $\mathrm{CH}_{3} \mathrm{CO}_{2} \mathrm{H}$ & \\
\hline Acetone & $\mathrm{CH}_{3} \mathrm{C}_{2} \mathrm{OH}_{3}$ & \\
\hline Chromic Acid & $\mathrm{H}_{2} \mathrm{CrO}_{4}$ & \\
\hline Cupric Sulfate & $\mathrm{CuSO}_{4} \cdot 5 \mathrm{H}_{2} \mathrm{O}$ & \\
\hline Ethanol & $\mathrm{C}_{2} \mathrm{H}_{5} \mathrm{OH}$ & \\
\hline Ethylene Glycol & $\mathrm{HOCH}_{2} \mathrm{CH}_{2} \mathrm{OH}$ & \\
\hline Hydrochloric Acid & $\mathrm{HCl}$ & \\
\hline Hydrofluoric Acid & $\mathrm{HF}$ & \\
\hline Hydrogen Peroxide & $\mathrm{H}_{2} \mathrm{O}_{2}$ & \\
\hline Nitric Acid & $\mathrm{HNO}_{3}$ & \\
\hline Oxalic Acid & $\mathrm{HO}_{2} \mathrm{CCO}_{2} \mathrm{H} \cdot 2 \mathrm{H}_{2} \mathrm{O}$ & \\
\hline Perchloric Acid & $\mathrm{HClO}_{4}$ & \\
\hline Phosphoric Acid & $\mathrm{H}_{3} \mathrm{PO}_{4}$ & \\
\hline Potassium Ferrocyanide & $\mathrm{K}_{4} \mathrm{Fe}_{2}\left(\mathrm{CN}_{6}\right.$ & \\
\hline Sodium Hydroxide & $\mathrm{NaOH}$ & \\
\hline Sulfuric Acid & $\mathrm{H}_{2} \mathrm{SO}_{4}$ & \\
\hline & & \\
\hline
\end{tabular}


WHC-EP-0172 Revision 1

Table 33. Building 327: Post-Irradiation Testing Laboratory (1953-1976). (Sheet 1 of 2)

\begin{tabular}{|c|c|c|}
\hline Compound name & Formula & Comments \\
\hline Acetone & $\mathrm{CH}_{3} \mathrm{C}_{2} \mathrm{OH}_{3}$ & \\
\hline Aluminum & $\mathrm{Al}$ & Fuel element constituent \\
\hline Benzene & $\mathrm{C}_{6} \mathrm{H}_{6}$ & \\
\hline Beryllium & $\mathrm{Be}$ & Fuel element constituent \\
\hline Boron Carbide & $\mathrm{B}_{4} \mathrm{C}$ & \\
\hline Butanol & $\mathrm{C}_{4} \mathrm{H}_{9} \mathrm{OH}$ & \\
\hline Carbon Tetrachloride & $\mathrm{CCl}_{4}$ & \\
\hline Cesium Nitrate & $\mathrm{CsNO}_{3}$ & \\
\hline Cesium Nitrite & $\mathrm{CsNO}_{2}$ & \\
\hline Chromic Acid & $\mathrm{H}_{2} \mathrm{CrO}_{4}$ & \\
\hline Ethanol & $\mathrm{C}_{2} \mathrm{H}_{5} \mathrm{OH}$ & \\
\hline Glycerol & $\begin{array}{l}\mathrm{CH}_{2} \mathrm{OHCHOHC} \\
\mathrm{H}_{2} \mathrm{OH}\end{array}$ & \\
\hline Gold & $\mathrm{Au}$ & \\
\hline Hydrofluoric Acid & $\mathrm{HF}$ & \\
\hline Hydrogen Peroxide & $\mathrm{H}_{2} \mathrm{O}_{2}$ & \\
\hline Isoamyl Acetate & $\mathrm{C}_{7} \mathrm{H}_{14} \mathrm{O}_{2}$ & \\
\hline Isopropyl Alcohol & $\mathrm{C}_{3} \mathrm{H}_{7} \mathrm{OH}$ & \\
\hline Kerosene (Inhibited) & $\begin{array}{l}\mathrm{C}_{10} \mathrm{H}_{22} \text { to } \mathrm{C}_{16} \mathrm{H}_{34}+ \\
\mathrm{Cl}_{2}{ }_{2} \mathrm{CCHCl}\end{array}$ & \\
\hline Lithium & $\mathrm{Li}$ & \\
\hline Methạnol & $\mathrm{CH}_{3} \mathrm{OH}$ & \\
\hline Methyl Ethyl Ketone & $\mathrm{CH}_{3} \mathrm{COC}_{2} \mathrm{H}_{5}$ & \\
\hline Mineral Oil & Light Hydrocarbons & \\
\hline Molybdenum & Mo & \\
\hline Nickel & $\mathrm{Ni}$ & \\
\hline Nitric Acid & $\mathrm{HNO}_{3}$ & \\
\hline Oxalic Acid & $\mathrm{HO}_{2} \mathrm{CCO}_{2} \mathrm{H} \cdot 2 \mathrm{H}_{2} \mathrm{O}$ & \\
\hline Perchloric Acid & $\mathrm{HClO}_{4}$ & \\
\hline Platinum & $\mathrm{Pt}$ & \\
\hline Potassium Hydroxide & $\mathrm{KOH}$ & \\
\hline Potassium Permanganate & $\mathrm{KMnO}_{4}$ & \\
\hline
\end{tabular}


Table 33. Building 327: Post-Irradiation Testing Laboratory (1953-1976). (Sheet 2 of 2)

\begin{tabular}{|l|l|l|}
\hline \multicolumn{1}{|c|}{ Compound name } & \multicolumn{1}{c|}{ Formula } & \multicolumn{1}{c|}{ Comments } \\
\hline Potassium Oxide & $\mathrm{K}_{2} \mathrm{O}$ & \\
\hline Silver & $\mathrm{Ag}$ & \\
\hline Silicon & $\mathrm{Si}$ & Fuel element constituent \\
\hline Sodium Hydroxide & $\mathrm{NaOH}$ & \\
\hline Sodium Phosphate & $\mathrm{Na}_{3} \mathrm{PO}_{4}$ & \\
\hline Stainless Steel & $\mathrm{FeNiCr}$ & Fuel element constituent \\
\hline Sulfuric Acid & $\mathrm{H}_{2} \mathrm{SO}_{4}$ & \\
\hline Tantalum & $\mathrm{Ta}$ & \\
\hline Trichloroethane & $\mathrm{CH}_{3} \mathrm{CCl}_{3}$ & \\
\hline Toluene & $\mathrm{C}_{6} \mathrm{H}_{5} \mathrm{CH}_{3}$ & \\
\hline Xylene & $\mathrm{C}_{6} \mathrm{H}_{4}\left(\mathrm{CH}_{3}\right)_{2}$ & \\
\hline Zirconium & $\mathrm{Zr}$ & Fuel element constituent \\
\hline
\end{tabular}


WHC-EP-0172 Revision 1

Table 34. Building 329: Radiochemical Laboratory (1952-1976). (Sheet 1 of 3)

\begin{tabular}{|c|c|c|}
\hline Compound name & Formula & Comments \\
\hline Acetone & $\mathrm{CH}_{3} \mathrm{C}_{2} \mathrm{OH}_{3}$ & \\
\hline $\begin{array}{l}\text { Aluminum Nitrate } \\
\text { Nonahydrate }\end{array}$ & $\mathrm{Al}\left(\mathrm{NO}_{3}\right)_{3} \cdot 9 \mathrm{H}_{2} \mathrm{O}$ & \\
\hline Ammonium Acetate & $\mathrm{NH}_{4} \mathrm{C}_{2} \mathrm{H}_{3} \mathrm{O}$ & \\
\hline Ammonium Chloride & $\mathrm{NH}_{4} \mathrm{Cl}$ & \\
\hline Ammonium Hydroxide & $\mathrm{NH}_{4} \mathrm{OH}$ & \\
\hline Ammonium Molybdate & $\left(\mathrm{NH}_{4}\right)_{2} \mathrm{MoO}_{4}$ & \\
\hline Ammomium Nitrate & $\mathrm{NH}_{4} \mathrm{NO}_{3}$ & \\
\hline Ammonium Sulfate & $\left(\mathrm{NH}_{4}\right)_{2} \mathrm{SO}_{4}$ & \\
\hline Ammonium Sulfite & $\mathrm{NH}_{4} \mathrm{HS}$ & \\
\hline Ammonium Thiocyanate & $\mathrm{NH}_{4} \mathrm{CNS}$ & \\
\hline Ammonium Thiosulfate & $\left(\mathrm{NH}_{4}\right)_{2} \mathrm{~S}_{2} \mathrm{O}_{3}$ & \\
\hline Antimony Chloride & $\mathrm{SbCl}_{3}$ & \\
\hline Antimony Nitrate & $\mathrm{Sb}\left(\mathrm{NO}_{3}\right)_{3}$ & \\
\hline Arsenic Trioxide & $\mathrm{As}_{2} \mathrm{O}_{3}$ & \\
\hline Benzene & $\mathrm{C}_{6} \mathrm{H}_{6}$ & \\
\hline Boric Acid & $\mathrm{H}_{3} \mathrm{BO}_{3}$ & \\
\hline Carbon Tetrachloride & $\mathrm{CCl}_{4}$ & \\
\hline Cerous Nitrate & $\mathrm{Ce}\left(\mathrm{NO}_{3}\right)_{3}$ & \\
\hline Cesium Chloride & $\mathrm{CsCl}$ & \\
\hline Cesium Nitrate & $\mathrm{CsNO}_{3}$ & \\
\hline Ethanol & $\mathrm{CH}_{3} \mathrm{CH}_{2} \mathrm{OH}$ & \\
\hline Ferrous Sulfamate & $\mathrm{Fe}\left(\mathrm{SO}_{3} \mathrm{NH}_{2}\right)_{2}$ & \\
\hline Ferrous Sulfate & $\mathrm{FeSO}_{4}$ & \\
\hline Hydrazine & $\mathrm{H}_{2} \mathrm{NNH}_{2} \cdot \mathrm{H}_{2} \mathrm{O}$ & \\
\hline Hydrobromic Acid & $\mathrm{HBr}$ & \\
\hline Hydrochloric Acid & $\mathrm{HCl}$ & \\
\hline Hydrofluoric Acid & HF & \\
\hline Hydrogen Sulfide & $\mathrm{H}_{2} \mathrm{~S}$ & \\
\hline
\end{tabular}


Table 34. Building 329: Radiochemical Laboratory

(1952-1976). (Sheet 2 of 3)

\begin{tabular}{|l|l|l|}
\hline \multicolumn{1}{|c|}{ Compound name } & \multicolumn{1}{c|}{ Formula } & Comments \\
\hline $\begin{array}{l}\text { Hydroxylamine } \\
\text { Hydrochloride }\end{array}$ & $\mathrm{NH}_{2} \mathrm{OH} \cdot \mathrm{HCl}$ & \\
\hline Lanthanum Chloride & $\mathrm{LaCl}_{3}$ & \\
\hline Lanthanum Nitrate & $\mathrm{La}\left(\mathrm{NO}_{3}\right)_{2}$ & \\
\hline Lead Nitrate & ${\mathrm{Pb}\left(\mathrm{NO}_{3}\right)_{2}}$ & \\
\hline Mandelic Acid & $\mathrm{C}_{6} \mathrm{H}_{5} \mathrm{CHOHCO}_{2} \mathrm{H}$ & \\
\hline Mercury & $\mathrm{Hg}$ & \\
\hline Neodymium Chloride & $\mathrm{NdCl}_{3}$ & \\
\hline Neodymium Nitrate & ${\mathrm{Nd}\left(\mathrm{NO}_{3}\right)_{3}}$ & \\
\hline Nitric Acid & $\mathrm{HNO}_{3}$ & \\
\hline Perchloric Acid & $\mathrm{HClO}_{4}$ & \\
\hline Phosphoric Acid & $\mathrm{H}_{3} \mathrm{PO}_{4}$ & \\
\hline Potassium Bisulfite & $\mathrm{K}_{2} \mathrm{~S}_{2} \mathrm{O}_{5}$ & \\
\hline Potassium Cyanide & $\mathrm{KCN}_{3}$ & \\
\hline Potassium Dichromate & $\mathrm{K}_{2} \mathrm{Cr}_{2} \mathrm{O}_{7}$ & \\
\hline Potassium Ferricyanide & $\mathrm{K}_{3} \mathrm{Fe}_{7}\left(\mathrm{CN}_{6}\right.$ & \\
\hline Potassium Ferrocyanide & $\mathrm{K}_{4} \mathrm{Fe}_{6}\left(\mathrm{CN}_{6} \cdot 3 \mathrm{H}_{2} \mathrm{O}\right.$ & \\
\hline Potassium Permanganate & $\mathrm{KMnO}_{4}$ & \\
\hline Potassium Persulfate & $\mathrm{K}_{2} \mathrm{~S}_{2} \mathrm{O}_{8}$ & \\
\hline Praseodymium Chloride & $\mathrm{PrCl}_{3}$ & \\
\hline Praseodymium Nitrate & $\mathrm{Pr}_{3}\left(\mathrm{NO}_{3}\right)_{3}$ & \\
\hline Selenium Chloride & $\mathrm{SeCl}_{4}$ & \\
\hline Selenium Nitrate & $\mathrm{Se}_{2}\left(\mathrm{NO}_{3}\right)_{4}$ & \\
\hline Silico Tungstic Acid & $\left.\mathrm{H}_{4} \mathrm{Si}_{4}\left(\mathrm{~W}_{3} \mathrm{O}_{10}\right)_{4}\right] \cdot$ & \\
\hline Silver Chloride & $26 \mathrm{H}_{2} \mathrm{O}$ & \\
\hline Silver Nitrate & $\mathrm{AgCl}$ & \\
\hline Sodium Acetate & $\mathrm{AgNO}_{3}$ & \\
\hline Sodium Bicarbonate & $\mathrm{NaC}_{2} \mathrm{H}_{3} \mathrm{O}_{2}$ & \\
\hline Sodium Bismuthate & $\mathrm{NaHCO}_{3}$ & \\
\hline Sodium Carbonate & $\mathrm{NaBiO}_{3}$ & \\
\hline & $\mathrm{Na}_{2} \mathrm{CO}_{3}$ & \\
\hline
\end{tabular}


WHC-EP-0172 Revision 1

Table 34. Building 329: Radiochemical Laboratory (1952-1976). (Sheet 3 of 3)

\begin{tabular}{|l|l|l|}
\hline \multicolumn{1}{|c|}{ Compound name } & \multicolumn{1}{c|}{ Formula } & Comments \\
\hline Sodium Hydroxide & $\mathrm{NaOH}$ & \\
\hline Sodium Hypochlorite & $\mathrm{NaOCl}$ & \\
\hline Sodium Iodide & $\mathrm{NaI}$ & \\
\hline Sodium Nitrate & $\mathrm{NaNO}_{3}$ & \\
\hline Sodium Sulfide & $\mathrm{Na}_{2} \mathrm{~S}$ & \\
\hline Sodium Thiocyanate & $\mathrm{NaSCN}$ & \\
\hline Sodium Thiosulfate & $\mathrm{Na}_{2} \mathrm{~S}_{2} \mathrm{O}_{3}$ & \\
\hline Strontium Chloride & $\mathrm{SrCl}_{2}$ & \\
\hline Strontium Nitrate & $\left.\mathrm{Sr}_{(} \mathrm{NO}_{3}\right)_{2}$ & \\
\hline Sulfuric Acid & $\mathrm{H}_{2} \mathrm{SO}_{4}$ & \\
\hline Thorium Chloride & $\mathrm{ThCl}_{4}$ & \\
\hline Thorium Nitrate & $\left.\mathrm{Th}_{4} \mathrm{NO}_{3}\right)_{4}$ & \\
\hline Tin Chloride & $\mathrm{SnCl}_{4}$ & \\
\hline Tin Nitrate & $\mathrm{Sn}_{4}\left(\mathrm{NO}_{3}\right)_{4}$ & \\
\hline Toluene & $\mathrm{C}_{6} \mathrm{H}_{5} \mathrm{CH}_{3}$ & \\
\hline Xylene & $\mathrm{C}_{6} \mathrm{H}_{4}\left(\mathrm{CH}_{3}\right)_{2}$ & \\
\hline Zinc Chloride & $\mathrm{ZnCl}_{2}$ & \\
\hline Zinc Nitrate & ${\mathrm{Zn}\left(\mathrm{NO}_{3}\right)_{2}}$ & \\
\hline
\end{tabular}


WHC-EP-0172 Revision 1

Table 35. Building 340: Radioactive Liquid Waste Storage and Railcar Loadout Facility (1950-1976).

\begin{tabular}{|l|l|l|}
\hline \multicolumn{1}{|c|}{ Compound name } & \multicolumn{1}{|c|}{ Formula } & \multicolumn{1}{c|}{ Comments } \\
\hline Sodium Hydroxide & $\mathrm{NaOH}$ & \\
\hline Sodium Nitrite & $\mathrm{NaNO}_{2}$ & \\
\hline
\end{tabular}


Table 36. Glossary of Product Names. (Sheet 1 of 3)

\begin{tabular}{|c|c|}
\hline Product name & Company \\
\hline Amberlite XE-270 & Rahom and Hass \\
\hline American Cyanamid S4058 Floc & American Cyanamid \\
\hline Antifoam 60 & General Electric \\
\hline Arseno III & Hatch Company \\
\hline Clayton Kerful Kleaner & Clayton Manufacturing Company \\
\hline Diversy Chemical 159 & Not Available \\
\hline Dow N-20 Floc & Dow Corning Corporation \\
\hline Dow XD 7889 Floc & Dow Corning Corporation \\
\hline Dow Antifoam B & Dow Corning Corporation \\
\hline Dowex 21K & Dow Chemical \\
\hline Dowex 50 & Dow Chemical \\
\hline Duolite ARC-359 & Diamond Shamrock Chemical Company \\
\hline Hyflo-Super-Cel & Manville Bulding Materials Corporation \\
\hline Immunuol 1468-2 & Harry Miller Corporation \\
\hline Ionac A-580 & Ionac Chemical Corporation \\
\hline Jasco Paint Stripper & Jasco Chemical Corporation \\
\hline Kelite 25E & Kelite Corporation \\
\hline Keraff & Not Available \\
\hline Kleeno Bowl & Penetone Corporation \\
\hline Mistron & Cyprus Minerals Corporation \\
\hline Oakite Clear Guard & Oakite Products Incorporated \\
\hline Oakite LSD & Oakite Products Incorporated \\
\hline Oakite Rust Stripper & Oakite Products Incorporated \\
\hline Oakite Swiff & Oakite Products Incorporated \\
\hline Orvus K & Procter and Gamble \\
\hline Saf-Tee Solvent F.O. 128 & Not Available \\
\hline Sani-Flush & Boyle Midway Incorporated \\
\hline Sparton DC 13 & Not Available \\
\hline Shell Spray Base & Shell Oil Company \\
\hline Shell E-2342 & Shell Oil Company \\
\hline
\end{tabular}


Table 36. Glossary of Product Names. (Sheet 2 of 3)

\begin{tabular}{|c|c|}
\hline Product name & Company \\
\hline Soltrol - 170 & Phillips Chemical Company \\
\hline Pace - S - Teen & Not Available \\
\hline Penvert 192 & Not Available \\
\hline Permutit SK & Ionac Chemical Corporation \\
\hline Perokleen & Not Available \\
\hline Spic and Span & Procter and Gamble \\
\hline Tide & Procter and Gamble \\
\hline Turco Fabrifilm & Turco Products Incorporated \\
\hline Turco EPO Strip & Turco Products Incorporated \\
\hline Turco EPO Strip NP & Turco Products Incorporated \\
\hline Turco 2822 & Turco Products Incorporated \\
\hline Turco 2844 & Turco Products Incorporated \\
\hline Turco 4306 B,C,\&D & Turco Products Incorporated \\
\hline Turco $4358-4 A$ & Turco Products Incorporated \\
\hline Turco $4501 \mathrm{~A}$ & Turco Products Incorporated \\
\hline Turco $4502 \mathrm{D}$ & Turco Products Incorporated \\
\hline Turco $4512 \mathrm{~A}$ & Turco Products Incorporated \\
\hline Turco 4518 & Turco Products Incorporated \\
\hline Turco 4605-8 & Turco Products Incorporated \\
\hline Turco 4669 & Turco Products Incorporated \\
\hline Tureo 4715 & Turco Products Incorporated \\
\hline Turco 4738 (Thin) & Turco Products Incorporated \\
\hline Turco T-5561 & Turco Products Incorporated \\
\hline Turco T-5589 & Turco Products Incorporated \\
\hline Turco Alkaline (Rust Remover) & Turco Products Incorporated \\
\hline Turco Deseal Zit 2 & Turco Products Incorporated \\
\hline Turco Plaudit & Turco Products Incorporated \\
\hline West Loade Degreaser & West Chemical Products \\
\hline
\end{tabular}


WHC-EP-0172 Revision 1

Table 36. Glossary of Product Names. (Sheet 3 of 3)

\begin{tabular}{|l|l|}
\hline \multicolumn{1}{|c|}{ Product name } & \multicolumn{1}{c|}{ Company } \\
\hline Wyandotte Kelvar & Wyandotte Chemical Products \\
\hline Wyandotte MF & Wyandotte Chemical Products \\
\hline Wyandotte P1075 & Wyandotte Chemical Products \\
\hline Wyandotte 1112 & Wyandotte Chemical Products \\
\hline Zelon 900 & Norton Company \\
\hline Zeolite AW-500 & Union Carbide Corporation \\
\hline
\end{tabular}




\subsection{REFERENCES}

1. W. I. Winters, L. Jenson, L. M. Sasaki, R. L Weiss, J. F. Keller, A. J. Schmidt, and M. G. Woodruff, Waste Characterization Plan for the Hanford Site Single-Shell Tanks, WHC-EP-0210, Westinghouse Hanford Company, Richland, Washington (May 1989).

2. DOE, Final Environmental Impact Statement Disposal of Hanford Defense High-Level, Transuranic and Tank Wastes, DOE/EIS-0113, U.S. Department of Energy, Washington, D.C. (December 1987).

3. U.S. Department of Energy, 10 CFR 962, Radioactive Waste: Byproducts Material Final Rule Federal Register, 52 FR 15937-15941 Office of the Federal Register, National Archives and Records Administration, Washington, D.C. (May 1, 1987).

4. 52 FR 35556 (September 22, 1987).

5. U.S.Department of Energy, Record of Decision for the Environmental Impact Statement for the Disposal of Hanford Defense High-Level, Transuranic and Tank Waste, 53 FR 12449, Office of the Federal Register, National Archives and Records Administration, Washington, D.C. (April 14, 1988).

6. EPA, DOE, and Ecology, 1989, Hanford Federal Facility Agreement and Consent Order, U.S. Environmental Protection Agency, U.S. Department of Energy, and Washington State Department of Ecology, Richland, Washington (May 1989).

7. Public Law, 84/11/09, "The 1984 Hazardous and Solid Waste Amendment," Resource Conversation and Recovery Act, PL-98-616, as amended (November 9, 1984) (cited as RCRA in text and information is from 40 CFR 261 Appendix VIII, "Hazardous Constituents," July 1, 1988).

8. Washington Hazardous Waste Management Act (RCW 70.105) ammended July 26, 1987.

9. The Atomic Energy Act of 1954, as amended, 42 U.S.C. 2011 et seq. (cited as AEA in text).

10. Public Law, Comprehensive Environmental Response Compensation and Liability Act of 1980, PL-98-45, as amended (July 12, 1983) (cited as CERCLA in text and information is from 40 CFR 302, Table 302.4, "List of Hazardous Substances and Reportable Quantities," July 1 , 1988).

11. OSHA, Toxic and Hazardous Substances, 29 CFR 1910.1000,Z-tables, Occupational Safety and Health Administration, Washington, D.C. (July 1, 1988) (cited as OSHA in text).

12. OSHA, 29 CFR 1910, Department of Labor: Air Contaminants--Proposed Rules, Federal Register, 53 FR 20960-et seq. Office of the Federal Register, National Archives and Records

Administration, Washington, D.C. (June 7, 1988) (Proposed revision and update exposure limits in OSHA's Z-tables at 29 CFR 1910.1000 and cited as OSHA in text).

13. Ecology, "Dangerous Waste Regulations," Washington Administrative Code 173-303, as amended, Washington State Department of Ecology (January 4,1989) (cited as WAC in text and information is from "Discarded Chemical Products List" and "Dangerous Waste Constituents"). 
14. Threshold Limit Values and Biological Exposure Indices for 1987-1988, American Conference of Governmental Industrial Hygienists, Cincinnati, Ohio (cited as Occupational Exposure Standards (OES) / Threshold Limit Values (TLV) in text).

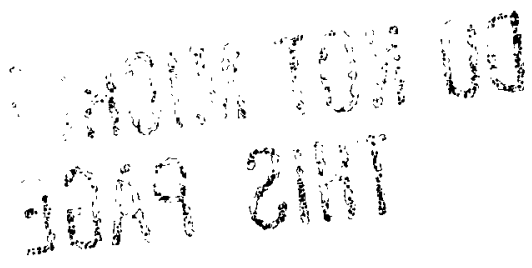


WHC-EP-0172 Revision 1

This page intentionally left blank.

\section{DO NOT MICROFILM THIS PAGE}




\section{DISTRIBUTION}

Number of copies

OFFSITE

1

ONSITE

5

9

73

\section{U.S. Department of Energy-Headquarters}

J. C. Lear

EM-442

\section{Germantown}

\section{Pacific Northwest Laboratory}
J. F. Keller
K6-55
L. G. Morgan
K2-35
P. F. Salter
P8-01
R. S. Wegeng
K6-28
M. G. Woodruff
K6-55

\section{U.S. Department of Energy-Richland Operations Office}

M. J. Anthony

G. J. Bracken

R. D. Freeberg

R. E. Gerton

J.C. Peschong

J. P. Sands

D. M. Smith

Reading Room (2)
A6-95

A6-80

A6-95

A6-80

A6-80

A6-95

A6-55

A1-65

Westinghouse Hanford Company

A. T. Alstad

H. Babad

J. W. Badden

D. G. Baide

D. E. Ball

M. S. Bensky

V. C. Boyles

D. R. Bratzel

J. W. Camman

H. F. Daugherty

A. J. DiLiberto

D. A. Dodd

F. D. Fisher

D. L. Flyckt

R. D. Fox

C. J. Geier

K. A. Giese

C. E. Goldberg
R1-51

B2-15

B2-19

R1-51

R1-17

R2-12

R1-51

T6-50

H4-54

R2-53

: $:$ R2-12

if

$\therefore \quad \mathrm{T} 5-12$

A2-26:

2x) $\mathrm{H} 4 \div 52$

H4-57

R2-12

R2-12 
Westinghouse Hanford Company (cont.)

V. W. Hall

B2-15

W. L. Johnson

H4-55

J. H. Kessner

T6-08

R. T. Kimura

R1-51

N. W. Kirch

R2-12

M. J. Klem (5)

R2-12

R. J. Landon

B2-19

M. J. Lauterbach

H4-55

R. E. Lerch

B2-35

V. D. Maupin

R1-51

H. E. McGuire

B2-35

K. J. Moss

R3-08

B. E. Opitz

R1-19

R. D. Pierce

R2-80

R. E. Raymond

R1-62

L. W. Roberts

F. A. Ruck

R2-80

L. M. Sasaki

H4-57

A. R. Schade

R2-12

E. H. Smith

B1-35

D. W. Strasser

B2-19

B. L. Vedder

R2-12

J. A. Voogd

B2-19

D. D. Wanner

R1-48

D. J. Washenfelder

R2-12

R. L. Weiss

R1-43

R. K. Welty

T6-50

R1-80

W. I. Winters

T6-50

D. D. Wodrich

R2-23

A. I. Wojtasek

R1-08

R. D. Wojtasek

B2-15

J. C. Womack

R2-18

Publications Services (11)

L8-15

Central Files (2)

L8-04

Environmental Data Management Center (6)

H4-22

\section{DO NOT MICROFILM THIS PAGE}

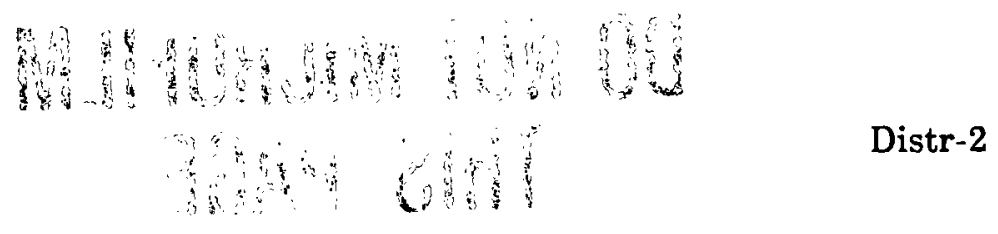

